\title{
Seds: The Spitzer Extended Deep Survey. Survey Design, Photometry, and Deep Irac Source Counts
}

\section{Citation}

Ashby, M. L. N., S. P. Willner, G. G. Fazio, J.-S. Huang, R. Arendt, P. Barmby, G. Barro, et al. 2013. “SEDS: THE SPITZER EXTENDED DEEP SURVEY. SURVEY DESIGN, PHOTOMETRY, AND DEEP IRAC SOURCE COUNTS." The Astrophysical Journal 769 (1): 80. https:// doi.org/10.1088/0004-637x/769/1/80.

\section{Permanent link}

http://nrs.harvard.edu/urn-3:HUL.InstRepos:41381811

\section{Terms of Use}

This article was downloaded from Harvard University's DASH repository, and is made available under the terms and conditions applicable to Other Posted Material, as set forth at http:// nrs.harvard.edu/urn-3:HUL.InstRepos:dash.current.terms-of-use\#LAA

\section{Share Your Story}

The Harvard community has made this article openly available. Please share how this access benefits you. Submit a story.

Accessibility 


\title{
SEDS: THE SPITZER EXTENDED DEEP SURVEY. SURVEY DESIGN, PHOTOMETRY, AND DEEP IRAC SOURCE COUNTS
}

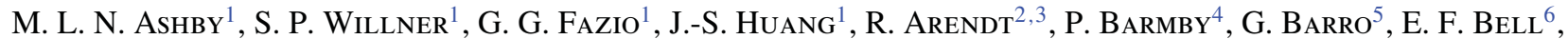 \\ R. Bouwens ${ }^{7}$, A. Cattaneo ${ }^{8}$, D. Croton ${ }^{9}$, R. Davé ${ }^{10}$, J. S. Dunlop ${ }^{11}$, E. Egami ${ }^{12}$, S. Faber ${ }^{5}$, K. Finlator ${ }^{13}$, N. A. Grogin ${ }^{14}$, \\ P. Guhathakurta ${ }^{5}$, L. Hernquist ${ }^{1}$, J. L. Hora ${ }^{1}$, G. Illingworth ${ }^{5}$, A. Kashlinsky ${ }^{2,15}$, A. M. KoeKemoer ${ }^{14}$, D. C. Koo $^{5}$,

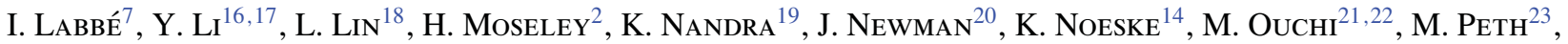 \\ D. Rigopoulou ${ }^{24,25}$, B. Robertson ${ }^{10,12}$, V. SARAJedini ${ }^{26}$, L. Simard ${ }^{27}$, H. A. SMith ${ }^{1}$, Z. WANG ${ }^{1}$, R. WechsLer ${ }^{28,29}$,

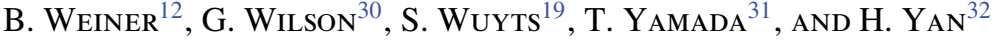 \\ ${ }^{1}$ Harvard-Smithsonian Center for Astrophysics, 60 Garden St., Cambridge, MA 02138, USA; mashby@cfa.harvard.edu \\ ${ }^{2}$ Observational Cosmology Laboratory, Code 665, Goddard Space Flight Center, Greenbelt, MD 20771, USA \\ ${ }^{3}$ CRESST, University of Maryland - Baltimore County, Baltimore, MD 21250, USA \\ ${ }^{4}$ University of Western Ontario, London, ON N6A 3K7, Canada \\ ${ }^{5}$ University of California Observatories/Lick Observatory and Department of Astronomy and Astrophysics \\ University of California Santa Cruz, 1156 High St., Santa Cruz, CA 95064, USA \\ ${ }^{6}$ Department of Astronomy, University of Michigan, 500 Church St., Ann Arbor, MI 48109, USA \\ ${ }^{7}$ Leiden Observatory, Leiden University, NL-2300 RA Leiden, The Netherlands \\ ${ }^{8}$ Aix Marseille Université, CNRS, Laboratoire d'Astrophysique de Marseille, UMR 7326, F-13388, Marseille, France \\ ${ }^{9}$ Centre for Astrophysics and Supercomputing, Swinburne University of Technology, P.O. Box 218 Hawthorn, VIC 3122, Australia \\ ${ }^{10}$ Department of Astronomy, University of Arizona, Tucson, AZ 85721, USA \\ ${ }^{11}$ Scottish Universities Physics Alliance, Institute for Astronomy, University of Edinburgh, Royal Observatory, Edinburgh, EH9 3HJ, UK \\ ${ }^{12}$ Steward Observatory, University of Arizona, 933 N. Cherry Ave, Tucson, AZ 85721, USA \\ ${ }^{13}$ Dark Cosmology Centre, Niels Bohr Institute, University of Copenhagen, Juliane Maries Vej 30, CK-2100 Copenhagen O, Denmark \\ ${ }^{14}$ Space Telescope Science Institute, 3700 San Martin Drive, Baltimore, MD 21218, USA \\ ${ }^{15}$ SSAI, Lanham, MD 20706, USA \\ ${ }^{16}$ Department of Physics \& Astronomy, Pennsylvania State University, 525 Davey Lab, University Park, PA 16802, USA \\ ${ }^{17}$ Institute for Gravitation and the Cosmos, Pennsylvania State University, 525 Davey Lab, University Park, PA 16802, USA \\ ${ }^{18}$ Institute of Astronomy \& Astrophysics, Academia Sinica, Taipei 106, Taiwan, ROC \\ ${ }^{19}$ Max-Planck-Institut für Extraterrestrische Physik, Postfach 1312, Giessenbachstrasse, D-85748 Garching, Germany \\ ${ }^{20}$ Department of Physics and Astronomy, University of Pittsburgh, 3941 O'Hara Street, Pittsburgh, PA 15260, USA \\ ${ }^{21}$ Institute for Cosmic Ray Research, University of Tokyo, 5-1-5 Kashiwanoha, Kashiwa, Chiba 277-8582, Japan \\ ${ }^{22}$ Kavli Institute for the Physics and Mathematics of the Universe (WPI), The University of Tokyo, 5-1-5 Kashiwanoha, Kashiwa, Chiba 277-8583, Japan \\ ${ }^{23}$ Department of Physics \& Astronomy, Johns Hopkins University, Baltimore, MD, USA \\ ${ }^{24}$ Astrophysics, University of Oxford, Keble Road, Oxford OX1 3RH, UK \\ ${ }^{25}$ Space Science \& Technology Department, Rutherford Appleton Laboratory, Chilton, Didcot, Oxfordshire OX11 0QX, UK \\ ${ }^{26}$ Department of Astronomy, University of Florida, Gainesville, FL 32611, USA \\ ${ }^{27}$ National Research Council of Canada, Herzberg Institute of Astrophysics, 5071 West Saanich Road, Victoria, British Columbia, Canada \\ ${ }^{28}$ Kavli Institute for Particle Astrophysics and Cosmology, Physics Department, Stanford University, Stanford, CA 94305, USA \\ ${ }^{29}$ SLAC National Accelerator Laboratory, Menlo Park, CA 94025, USA \\ ${ }^{30}$ Department of Physics and Astronomy, University of California Riverside, Riverside, CA 92521, USA \\ ${ }^{31}$ Astronomical Institute, Tohoku University, Japan \\ ${ }^{32}$ Department of Physics \& Astronomy, University of Missouri-Columbia, Columbia, MO 65211, USA \\ Received 2012 October 20; accepted 2013 April 3; published 2013 May 6
}

\begin{abstract}
The Spitzer Extended Deep Survey (SEDS) is a very deep infrared survey within five well-known extragalactic science fields: the UKIDSS Ultra-Deep Survey, the Extended Chandra Deep Field South, COSMOS, the Hubble Deep Field North, and the Extended Groth Strip. SEDS covers a total area of $1.46 \mathrm{deg}^{2}$ to a depth of $26 \mathrm{AB}$ mag $(3 \sigma)$ in both of the warm Infrared Array Camera (IRAC) bands at 3.6 and $4.5 \mu \mathrm{m}$. Because of its uniform depth of coverage in so many widely-separated fields, SEDS is subject to roughly $25 \%$ smaller errors due to cosmic variance than a single-field survey of the same size. SEDS was designed to detect and characterize galaxies from intermediate to high redshifts $(z=2-7)$ with a built-in means of assessing the impact of cosmic variance on the individual fields. Because the full SEDS depth was accumulated in at least three separate visits to each field, typically with six-month intervals between visits, SEDS also furnishes an opportunity to assess the infrared variability of faint objects. This paper describes the SEDS survey design, processing, and publicly-available data products. Deep IRAC counts for the more than 300,000 galaxies detected by SEDS are consistent with models based on known galaxy populations. Discrete IRAC sources contribute $5.6 \pm 1.0$ and $4.4 \pm 0.8 \mathrm{nW} \mathrm{m}^{-2} \mathrm{sr}^{-1}$ at 3.6 and $4.5 \mu \mathrm{m}$ to the diffuse cosmic infrared background (CIB). IRAC sources cannot contribute more than half of the total CIB flux estimated from DIRBE data. Barring an unexpected error in the DIRBE flux estimates, half the CIB flux must therefore come from a diffuse component.
\end{abstract}

Key words: galaxies: high-redshift - infrared: galaxies - surveys

Online-only material: color figures, machine-readable tables 


\section{INTRODUCTION}

The Spitzer Extended Deep Survey (SEDS) is an unbiased deep survey carried out by the Spitzer Space Telescope (Werner et al. 2004). The survey consists of integrations to $12 \mathrm{hr}$ per pointing at 3.6 and $4.5 \mu \mathrm{m}$ with Spitzer's Infrared Array Camera (IRAC; Fazio et al. 2004b) in five well-studied fields (E-GOODS-S, E-GOODS-N, EGS, UDS, and COSMOS / UltraVISTA) with a goal of covering a total of one square degree. To study variability, each SEDS field was visited three times, obtaining four hours/pointing on each visit. Visits were typically separated by six months. SEDS required a total of $2103 \mathrm{hr}$, making it the single largest Exploration Science Program carried out by Spitzer to date.

SEDS was designed from the outset to carry out a census of stellar mass and black holes as a function of cosmic time reaching back to the era of reionization. SEDS was also designed to measure galaxy clustering over a wide redshift range to provide a critical link between galaxies and their dark matter halos. These observations will be critical for testing galaxy formation models in the early universe. These outcomes are made possible by IRAC's unique ability to sample the rest-frame visible light of galaxies and active galactic nuclei (AGNs) at high redshift. The resulting broad-band spectral energy distributions can be combined with stellar population models to give stellar masses and ages. Specifically, the SEDS design goals were to detect galaxies down to $\sim 5 \times 10^{9} M_{\odot}$ at $z=6$. This sensitivity is necessary to robustly measure $M^{*}$ at that redshift, i.e., to detect the galaxies that dominate the global stellar mass density. Based on the cosmological hydrodynamic simulations of Davé et al. (2006), specifically their favored momentum-driven winds model, this is achieved at $26 \mathrm{AB}$ mag in the $3.6 \mu \mathrm{m}$ band. The SEDS depth was set accordingly. The SEDS design also enables several additional studies, including (1) galaxy evolution in the redshift range $z=1-6$, (2) obscured AGN growth at high redshift, (3) AGN variability, and (4) measurement of the cosmic infrared background (CIB) spatial fluctuations. SEDS explores the universe at a depth never before achieved over such a wide area at mid-infrared wavelengths. The depth and area coverage achieved by SEDS is compared to that from other major Spitzer/ IRAC surveys in Figure 1.

This paper is organized as follows: Section 2 describes the five SEDS fields with particular attention to prior IRAC imaging incorporated into the final SEDS data products. Section 3 discusses the details of the SEDS observing strategy and data reduction, and Section 4 describes the source identification, photometry, and validation. Section 5 describes the SEDS catalogs. In Section 6 we present preliminary results, including deep mid-IR number counts and the infrared color distribution of IRAC-detected galaxies. Section 7 summarizes these results. All magnitudes are stated in the AB system.

\section{THE FIVE SEDS FIELDS}

Because a key driver of SEDS science is the need to derive stellar mass estimates and galaxy stellar mass functions, it was vital to select fields with substantial quantities of deep broadband photometry. Of special importance are the availability of near-IR and visible imaging deep enough to match the IRAC observations reported here. An exhaustive search of potential target fields led to a choice of five widely separated premiere deep-sky survey regions. These are the $30^{\prime} \times 30^{\prime}$ Extended GOODS-South (aka the GEMS field, hereafter ECDFS; Rix et al. 2004; Castellano et al. 2010), the $30^{\prime} \times 30^{\prime}$ Extended

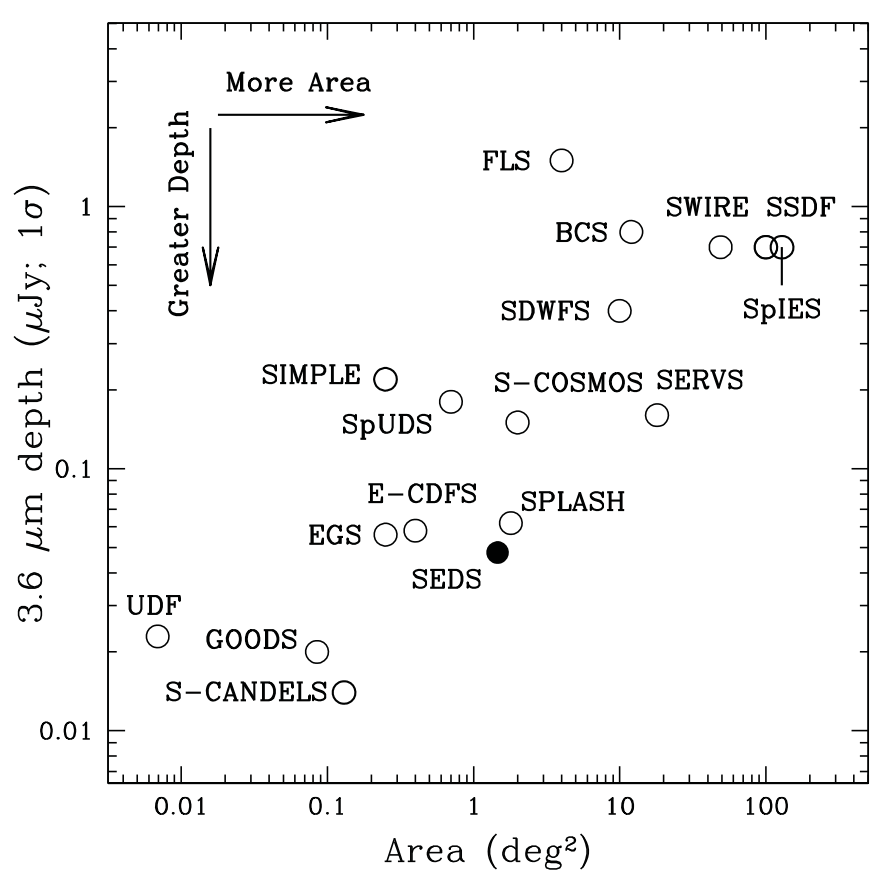

Figure 1. Comparison of SEDS $3.6 \mu \mathrm{m}$ depth and total area (solid circle) to other major Spitzer/IRAC extragalactic surveys (open circles). Points indicate either achieved or SENS-PET $1 \sigma$ point-source sensitivities for GOODS (Great Observatories Origins Deep Survey), the EGS (Extended Groth Strip), E-CDFS (Extended Chandra Deep Field South), SpUDS (Spitzer Public Legacy Survey of UKIDSS Ultra-Deep Survey), SCOSMOS (Spitzer Deep Survey of HST COSMOS 2-Degree ACS Field), SERVS (Spitzer Extragalactic Representative Volume Survey), BCS (Blanco Cluster Survey), SWIRE (Spitzer Wide-area Infrared Extragalactic Survey), the FLS (Spitzer First-Look Survey), SDWFS (Spitzer Deep, Wide-Field Survey), the SSDF (SPT-Spitzer Deep Field), S-CANDELS (Spitzer-CANDELS), the UDF (Ultra-Deep Field), SIMPLE (the Spitzer IRAC/MUSYC Public Legacy in E-CDFS), SpIES (Spitzer-IRAC Equatorial Survey), and SPLASH (Spitzer Large-Area Survey with HyperSuprime-Cam).

GOODS-North (HDFN; Giavalisco et al. 2004; Wang et al. 2010; Hathi et al. 2012; Lin et al. 2012), the $50^{\prime} \times 50^{\prime}$ UKIDSS Ultra-Deep Survey (UDS; aka the Subaru/XMM Deep Field, Ouchi et al. 2001; Lawrence et al. 2007), a $10^{\prime} \times 60^{\prime}$ region within the Extended Groth Strip (EGS; Davis et al. 2007; Bielby et al. 2012), and a $10^{\prime} \times 60^{\prime}$ strip within the UltraVista deep survey of the larger COSMOS field (Scoville et al. 2007b; Koekemoer et al. 2007; McCracken et al. 2012).

The five fields are distributed in ecliptic longitude and declination to permit ground-based followup from both hemispheres. Each field has its own combination of strengths; no two are alike but each has an extensive suite of supporting data from both ground- and space-based observatories throughout the optical and near-IR bands. The deep near-IR imaging taken with Hubble Space Telescope (HST)/WFC3 by Cosmic Assembly Near-Infrared Deep Extragalactic Legacy Survey (CANDELS; Grogin et al. 2011; Koekemoer et al. 2011) are an especially important component of these supporting data, because they cover a portion of each SEDS field in multiple infrared bands at significantly higher spatial resolution than IRAC. Some fields also have additional IRAC data that were not used either because they were relatively shallow or covered only limited areas. Notes on the IRAC observations of individual SEDS fields follow (all IRAC data sets used in this paper are listed in Table 1):

The first IRAC observations of the UDS field were the shallow coverage obtained by SWIRE $(4 \times 30 \mathrm{~s}$ exposures, reaching $23.7 \mathrm{mag}(1 \sigma)$ at $4.5 \mu \mathrm{m}$; Lonsdale et al. 2003, 2004; 
Table 1

The Five SEDS Fields

\begin{tabular}{|c|c|c|c|}
\hline Field & $\mathrm{PID}^{\mathrm{a}}$ & Epoch & $3.6 \mu \mathrm{m}, 4.5 \mu \mathrm{m}$ BCDs Used \\
\hline \multicolumn{4}{|c|}{ UDS $\left(2: 18: 00,-5: 10: 17,0.32 \mathrm{deg}^{2}\right)$} \\
\hline & 142 & 2004 Jul 27-28 & $548,597^{b}$ \\
\hline & 40021 & 2008 Jan 26-29 & 3640,3457 \\
\hline & 61041 & 2009 Sep 8-23 & 5255,5256 \\
\hline & 61041 & 2010 Feb 13-Mar 2 & 5328,5328 \\
\hline & 61041 & 2010 Sep 22-Oct 13 & 5436,5436 \\
\hline \multicolumn{4}{|c|}{ ECDFS $\left(3: 32: 20,-27: 37: 20,0.35 \mathrm{deg}^{2}\right)$} \\
\hline & 81 & 2004 Feb 16 & 167,146 \\
\hline & 194 & 2004 Feb 8-16 & $1724,1723^{\mathrm{c}}$ \\
\hline & 194 & 2004 Aug 12-18 & $1632,1632^{\mathrm{c}}$ \\
\hline & 20708 & 2005 Aug 19-23 & 1943,1872 \\
\hline & 20708 & 2006 Feb 6-11 & 1899,1944 \\
\hline & 30866 & 2007 Feb 15 & 1200,1080 \\
\hline & 60022 & 2010 Sep 20-Oct 4 & 4752,4588 \\
\hline & 60022 & 2011 Mar 26-Apr 3 & 4596,4752 \\
\hline & 60022 & 2011 Oct $10-$ Oct 20 & 4717,4552 \\
\hline & 70204 & 2011 Mar 17-Apr 7 & 5184,5128 \\
\hline \multicolumn{4}{|c|}{ COSMOS (10:00:30, +2:10:00, $\left.0.19 \mathrm{deg}^{2}\right)$} \\
\hline & 20070 & 2005 Dec 30-2006 Jan 2 & 1259,1253 \\
\hline & 61043 & 2010 Jan 25-Feb 4 & 3672,3672 \\
\hline & 61043 & 2010 Jun $10-28$ & 3164,3140 \\
\hline & 61043 & 2011 Jan 30-Feb 6 & 3180,3196 \\
\hline \multicolumn{4}{|c|}{ HDFN $\left(12: 36: 12,+62: 14: 12,0.25 \mathrm{deg}^{2}\right)$} \\
\hline & 81 & 2004 May 26-27 & 215,178 \\
\hline & 169 & 2004 May 16-26 & $2609,2609^{c}$ \\
\hline & 169 & 2004 Nov $17-25$ & $2447,2447^{\mathrm{c}}$ \\
\hline & 169 & 2005 Nov 25 & $114,114^{\mathrm{c}}$ \\
\hline & 20218 & 2005 Nov 28-Dec 9 & 200,200 \\
\hline & 20218 & 2006 Jun $2-3$ & 200,200 \\
\hline & 61040 & 2010 May $12-29$ & 4895,4896 \\
\hline & 61040 & 2011 Feb 28-Mar 13 & 5440,5440 \\
\hline & 60140 & 2011 May 22-Jun 2 & 5208,4896 \\
\hline \multicolumn{4}{|c|}{ EGS $\left(14: 19: 38,+52: 25: 47,0.35 \mathrm{deg}^{2}\right)$} \\
\hline & 8 & 2003 Dec 21-28 & $988,969^{c}$ \\
\hline & 8 & 2004 Jun 28-Jul 3 & $1027,989^{c}$ \\
\hline & 8 & 2006 Mar 28-29 & $117,24^{\mathrm{c}}$ \\
\hline & 41023 & 2008 Jan 24-25 & 726,726 \\
\hline & 41023 & 2008 Jul 21-23 & 726,726 \\
\hline & 61042 & 2010 Feb 5-16 & 4056,4056 \\
\hline & 61042 & 2010 Aug 4-19 & 4021,4056 \\
\hline & 61042 & 2011 Feb 10-22 & 3970,4048 \\
\hline
\end{tabular}

Notes. SEDS field positions and areas. Areas are those covered in both IRAC channels to a depth of at least $10 \mathrm{ks}$ by the projects listed.

a Spitzer Program Identification Number.

b $30 \mathrm{~s}$ frames.

c $200 \mathrm{~s}$ frames.

PID 142). Later, to match the depths of the newly-available near-IR photometry from the UKIRT Infrared Deep Sky Survey (UKIDSS; Lawrence et al. 2007) this field was surveyed a second time with IRAC as part of the Spitzer Public Legacy survey of the UKIDSS (SpUDS; Caputi et al. 2011). SpUDS covered a roughly $1 \mathrm{deg}^{2}$ area in all four of the IRAC bands available during the cryogenic mission to significantly deeper levels (e.g., reaching $24.7 \mathrm{mag}(1 \sigma)$ at $4.5 \mu \mathrm{m}$ ).

The SEDS observations of the UDS were carried out in three epochs from 2009 to 2010 (Table 1). A $0.40 \mathrm{deg}^{2}$ region within SpUDS was covered during each SEDS visit with a total exposure time of roughly $72 \times 100 \mathrm{~s}$ in each pixel of the resulting mosaic (Figure 2). When the full-depth UDS mosaic was assembled, it incorporated all the coextensive SWIRE and SpUDS exposures to reach the survey goal of $12 \mathrm{hr}$ total integration per pixel. The full-depth SEDS mosaics and coverage maps of the UDS are shown in Figures 3-6. The SEDS IRAC coverage is irregular because a portion of the northeast corner was avoided to prevent the bright stars there from introducing strong short-term latents into the exposures. A small area to the south was instead observed (the peninsula visible in Figures 5 and 6) because it was known to contain $z=6 \mathrm{Ly} \alpha$ emitting galaxies. Users should be aware of this selection bias when using SEDS data from the peninsula.

The ECDFS has been observed by a number of Spitzer programs starting at the very beginning of the mission because of the compelling depth and wavelength coverage of complementary observations, including HST/ACS coverage from GEMS (Rix et al. 2004). The IRAC observations have been taken at a variety of depth/area combinations. The earliest IRAC mosaics come from the Great Observatories Origins Deep Survey (GOODS) project, and cover a small region to roughly $26 \mathrm{mag}(3 \sigma)$ at 3.6 and $4.5 \mu \mathrm{m}$. In Spitzer Cycle 2, a much larger $1600 \mathrm{arcmin}^{2}$ region surrounding the GOODS region was covered by the IRAC/MuSYC Public Legacy Survey in the Extended CDF-South (SIMPLE; Damen et al. 2011). An additional program (Labbé et al. 2012) was carried out to get deep coverage of the two UDF NICMOS parallel fields with IRAC, resulting in an additional $\sim 27 \mathrm{hr}$ integration but covering a very small region, just slightly larger than a single IRAC field of view (FOV).

The SEDS imaging, which covers a subregion of SIMPLE, was carried out in three separate visits in 2010 and 2011. The SEDS coverage was designed to avoid the GOODS IRAC pointings, which were already deeper than SEDS in any case. Because of the pre-existing coverage, SEDS only obtained roughly $108 \times 100 \mathrm{~s}$ exposures at each pointing, this amount being sufficient to reach the target $12 \mathrm{hr}$ total integration time. Finally, a Cycle 7 program (PID 70204) was carried out to image a very small region of the ECDFS but very deeply, accumulating an additional $75 \mathrm{hr}$ in dithered $100 \mathrm{~s}$ exposures in approximately two IRAC FOVs within SEDS. The full-depth SEDS mosaics and coverage maps, which are combined with data from GOODS, SIMPLE, and PID 72004, are shown in Figures 7-10.

The COSMOS field subtends roughly $2 \mathrm{deg}^{2}$ overall, located so as to take advantage of a local minimum in the far-infrared background $\left(0.9 \mathrm{MJy} \mathrm{sr}^{-1}\right.$ at $\left.100 \mu \mathrm{m}\right)$. High-resolution HST/ ACS F814W imaging is available for the entire field (Scoville et al. 2007a; Koekemoer et al. 2007). COSMOS was first imaged by Spitzer in all four IRAC bands by S-COSMOS (Sanders et al. 2007). S-COSMOS consisted of dithered/ overlapping $12 \times 100 \mathrm{~s}$ exposures, achieving sensitivities of 23.9 and $23.3 \mathrm{mag}(5 \sigma)$ at 3.6 and $4.5 \mu \mathrm{m}$, respectively, over $2.3 \mathrm{deg}^{2}$.

The SEDS observations in S-COSMOS were arranged to cover a narrow strip roughly $10^{\prime} \times 1^{\circ}$ oriented $\mathrm{N}-\mathrm{S}$. The coverage was sited to coincide with very deep ground-based $Y J H K_{S}$ coverage obtained by McCracken et al. (2012) from the VISTA telescope (UltraVISTA). The SEDS data were accumulated in three visits in 2010 and 2011 (Table 1). The full-depth SEDS COSMOS mosaics and coverage maps, which incorporate the S-COSMOS exposures, are shown in Figures 11-14. The deep coverage is bordered by a relatively wide "crust" of intermediate-depth coverage. The crust arises from the tight 


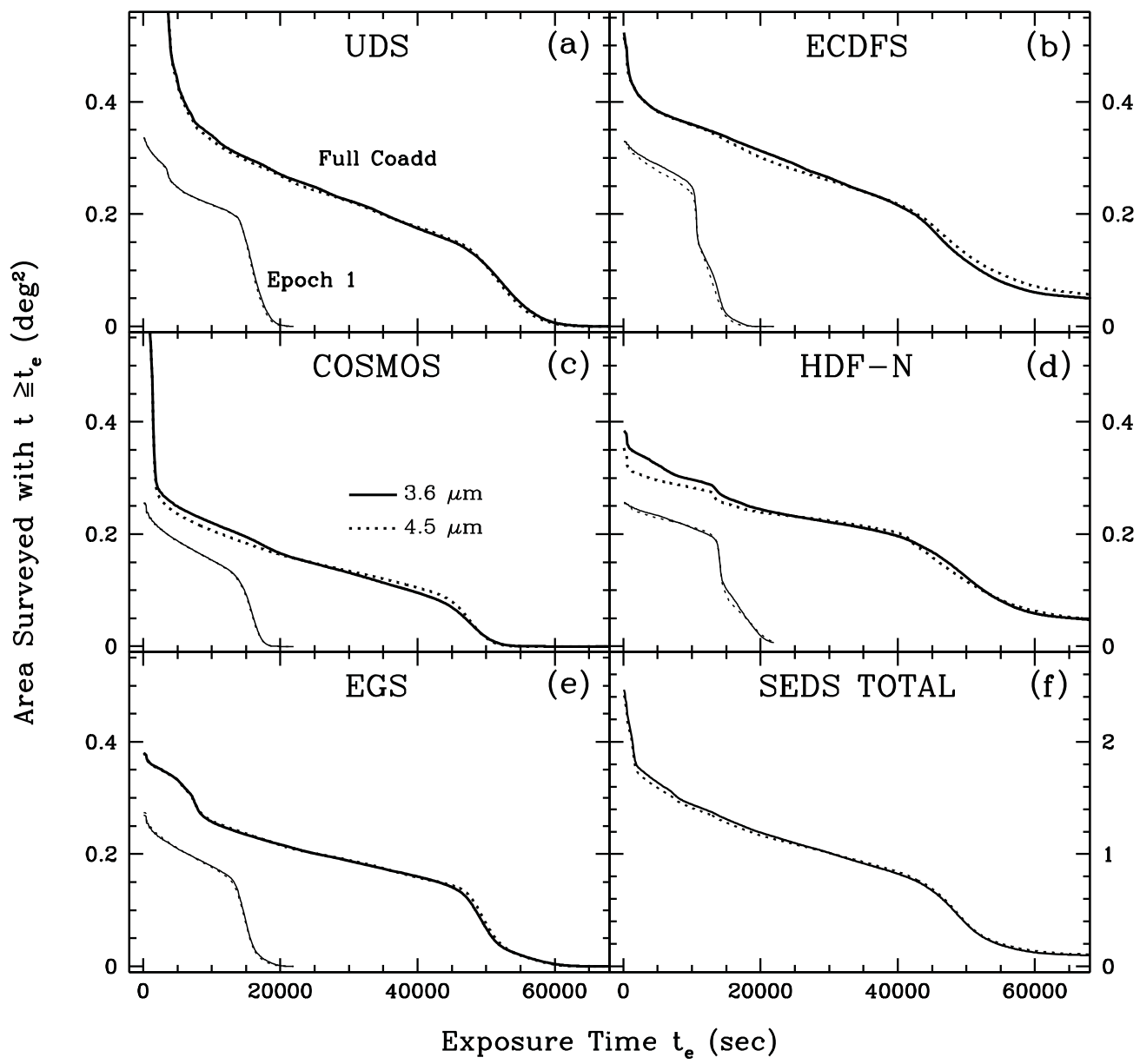

Figure 2. Cumulative area coverage as a function of exposure time for SEDS, including other, earlier observations (Table 1). The solid and dotted lines correspond respectively to the 3.6 and $4.5 \mu \mathrm{m}$ bands. Each panel shows the coverage for the first SEDS epoch (lower curves) together with that for the full-depth mosaics that combine all data (Table 1) for each field. Panel (f) shows the coverage summed over all five SEDS fields. The nominal SEDS depth was $12 \mathrm{hr}$ (43.2 ks); source extraction and photometry was only performed on regions having at least $10 \mathrm{ks}$ depth.

spacecraft roll angle constraints that prevail for this low-ecliptic latitude field.

The HDFN was first observed by IRAC in Spitzer Cycle 1 by the GOODS team (Giavalisco et al. 2004), who used a narrow and deep configuration to cover the multiband data originally obtained with HST (Williams et al. 1996). Those first IRAC observations consisted of dithered $200 \mathrm{~s}$ exposures arranged in a pair of $2 \times 2$ maps with a deeper region of overlapping coverage in the center. Subsequently, a wider $300 \mathrm{arcmin}^{2}$ region surrounding the GOODS coverage was observed in Cycle 2 with $100 \mathrm{~s}$ frames, reaching $24.5 \mathrm{mag}$ at $3.6 \mu \mathrm{m}$.

SEDS was the next program to observe this region, covering it three times in 2010 and 2011. Each SEDS visit covered a region roughly $30^{\prime} \times 30^{\prime}$ overlapping with the flanking-field coverage from Cycle 2 but avoiding the GOODS observations. The full-depth SEDS mosaics and coverage maps (Figures 15-18) incorporate the data from both previous programs. The coverage matches the footprint of the $J H K$ survey conducted with CFHT/ WIRCAM (Wang et al. 2010; Lin et al. 2012).

The EGS, like the other four regions surveyed by SEDS, benefits from a substantial array of multiwavelength imaging (Davis et al. 2007). The first substantial Spitzer/IRAC imaging, reaching $50 \%$ completeness at $23.4 \mu \mathrm{Jy}$ at $3.6 \mu \mathrm{m}$, was carried out by Barmby et al. (2008) along a narrow $2.3 \times 12^{\prime}$ strip placed to coincide with preexisting imaging acquired by HST's Advanced Camera for Surveys (ACS). These observations
(PID 8, Fazio, Rieke, \& Wright) were obtained in two separate visits in 2003 and 2004. They were augmented by two additional IRAC visits in 2008 (PID 41023, PI: Nandra) that broadened the central portion $(\sim 1 \mathrm{deg})$ of the long strip so as to provide much better overlap between the IRAC coverage and very deep (800 ks) X-ray imaging by Chandra.

The SEDS observations of the EGS cover the central degree, coincident with the IRAC imaging from both Barmby et al. (2008) and the additional observations from Cycle 4 as well as the multiband HST imaging from CANDELS. The SEDS imaging was carried out in three visits during 2010 and 2011 (Table 1). The full-depth SEDS mosaics and coverage maps incorporate all coextensive data from the earlier programs. They are shown in Figures 19-22.

\section{OBSERVATIONS AND DATA REDUCTION}

\subsection{Mapping Strategy}

The SEDS observing strategy was governed by the need to maximize the area covered with deep integrations in each of the five fields. It was also necessary to cover each field fully on separate visits so as to enable the planned variability science. Each field was visited three times; the visits (referred to below as epochs) were separated by six months except that the intervals for HDFN were nine and three months. Table 1 lists the epochs for each field. For each field, the coverage was very similar in 


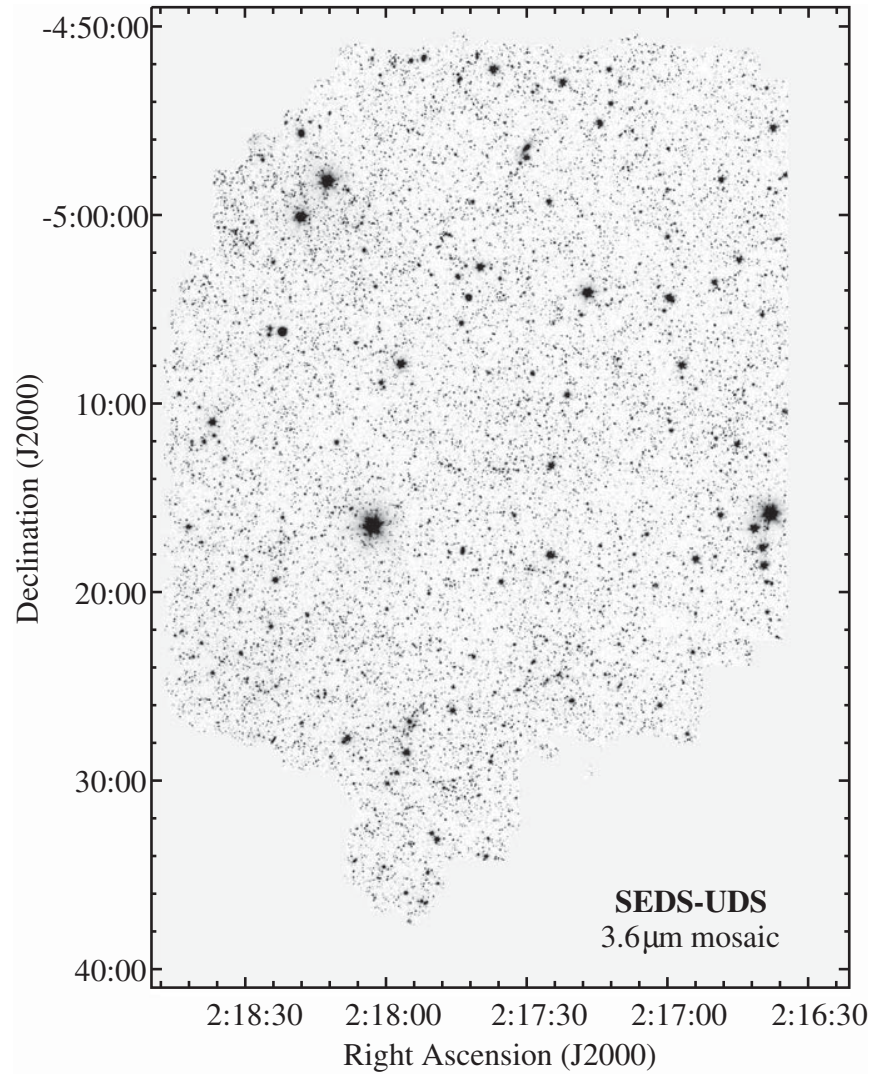

Figure 3. The full-depth SEDS $3.6 \mu \mathrm{m}$ mosaic in the UDS field (three coadded epochs, plus the coextensive imaging taken during the cryogenic mission and listed in Table 1). The image stretch ranges from -0.002 (white) to $0.05 \mathrm{MJy} \mathrm{sr}^{-1}$ (black).

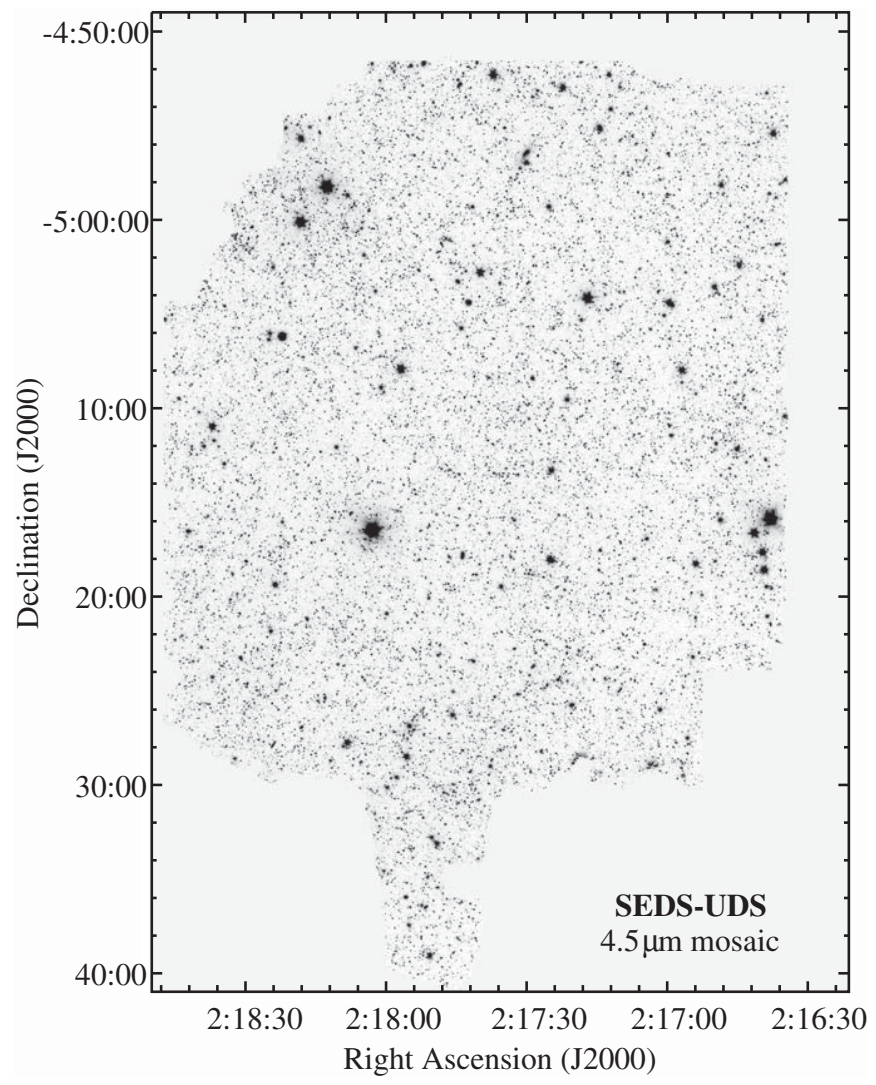

Figure 4. As Figure 3, but showing the SEDS $4.5 \mu \mathrm{m}$ mosaic of the UDS. The stretch ranges from -0.002 to $0.05 \mathrm{MJy} \mathrm{sr}^{-1}$.

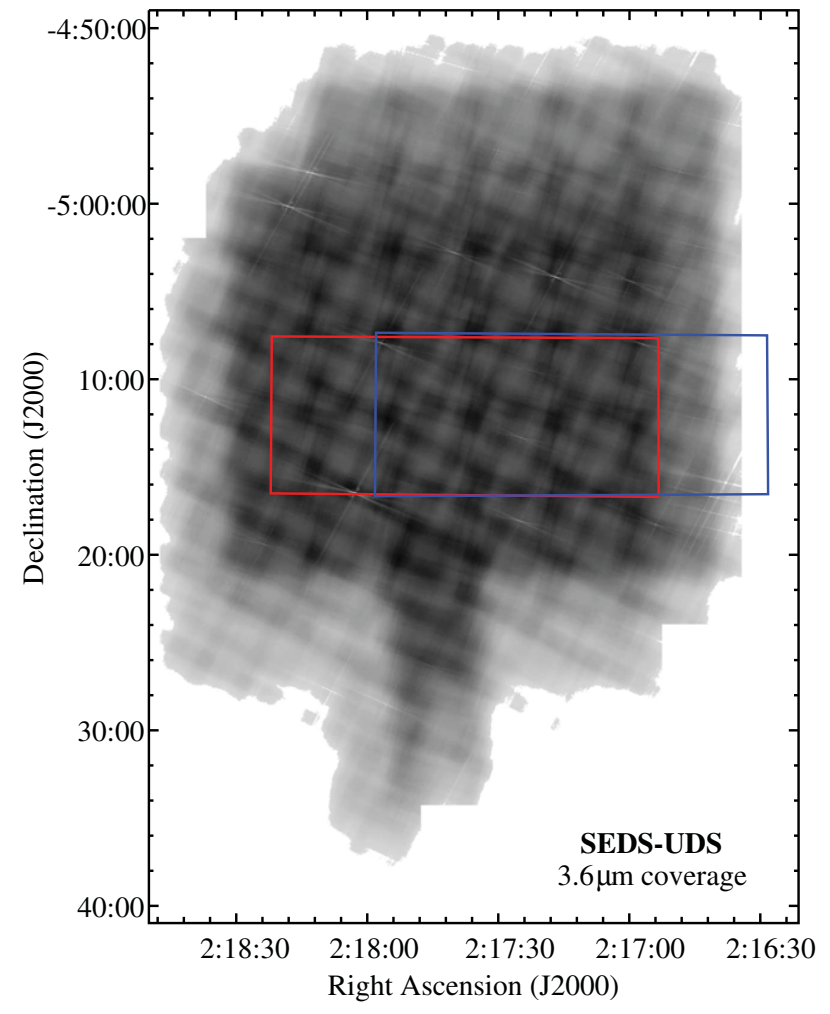

Figure 5. Total SEDS IRAC $3.6 \mu \mathrm{m}$ coverage map in the UDS field corresponding to the mosaic shown in Figure 3. The stretch ranges from 0 (white) to $70 \mathrm{ks}$ (black); the deepest coverage in this field is $65.1 \mathrm{ks}$. The total area observed with at least five $100 \mathrm{~s}$ exposures is $0.41 \mathrm{deg}^{2}$. The red and blue rectangles respectively indicate the areas surveyed with the WFC3 and ACS instruments by CANDELS.

(A color version of this figure is available in the online journal.)

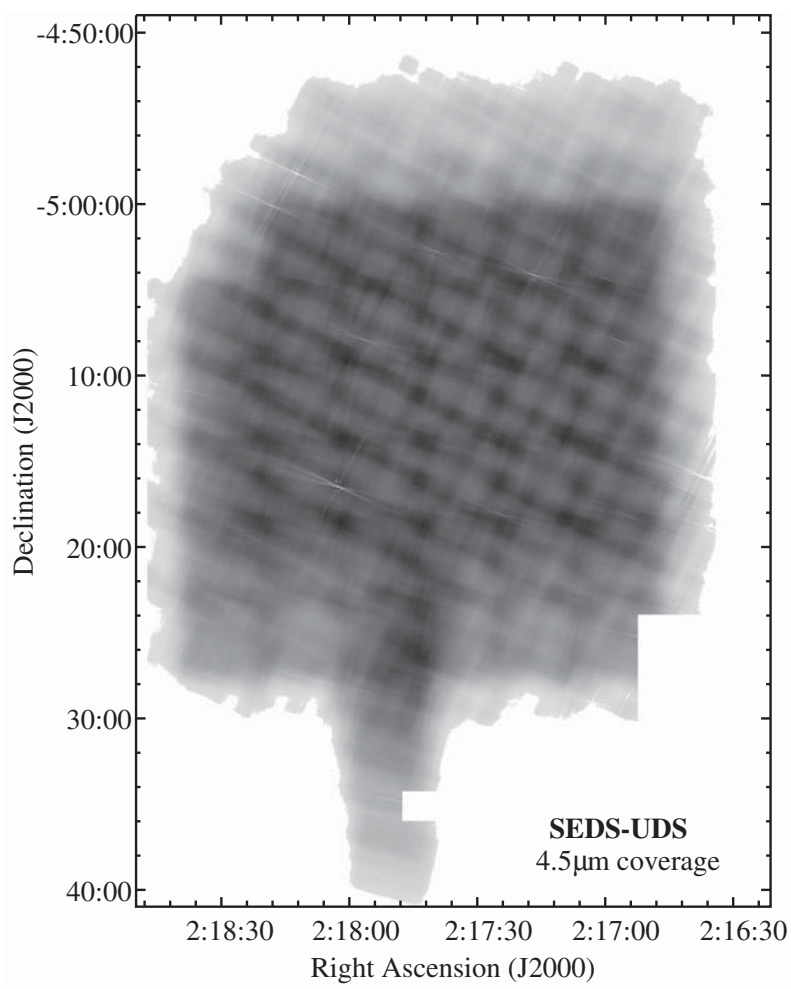

Figure 6. As Figure 5, but showing the SEDS $4.5 \mu \mathrm{m}$ coverage map of the UDS field. The stretch ranges from 0 (white) to $70 \mathrm{ks}$ (black). The deepest coverage in this field is $61.9 \mathrm{ks}$. The total area observed with at least five $100 \mathrm{~s}$ exposures is $0.40 \mathrm{deg}^{2}$. 


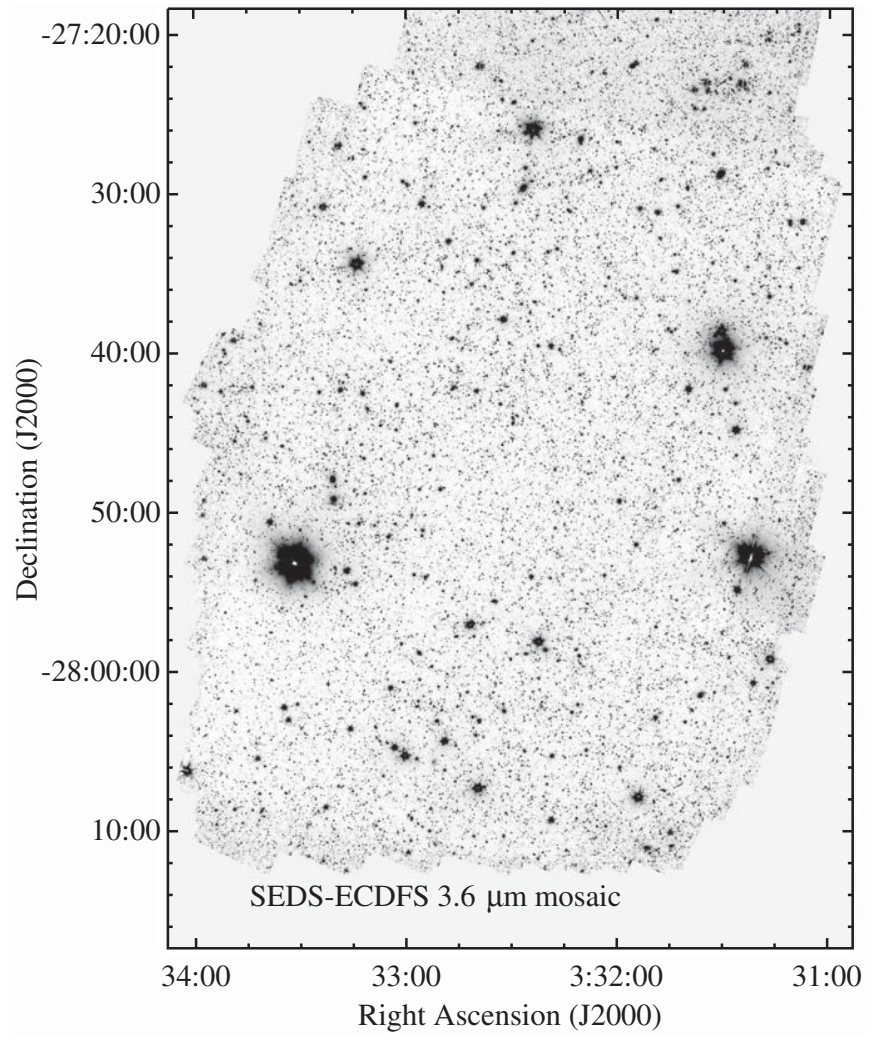

Figure 7. As Figure 3, but showing the SEDS IRAC $3.6 \mu \mathrm{m}$ mosaic of the ECDFS. The stretch ranges from 0 to $0.05 \mathrm{MJy} \mathrm{sr}^{-1}$.

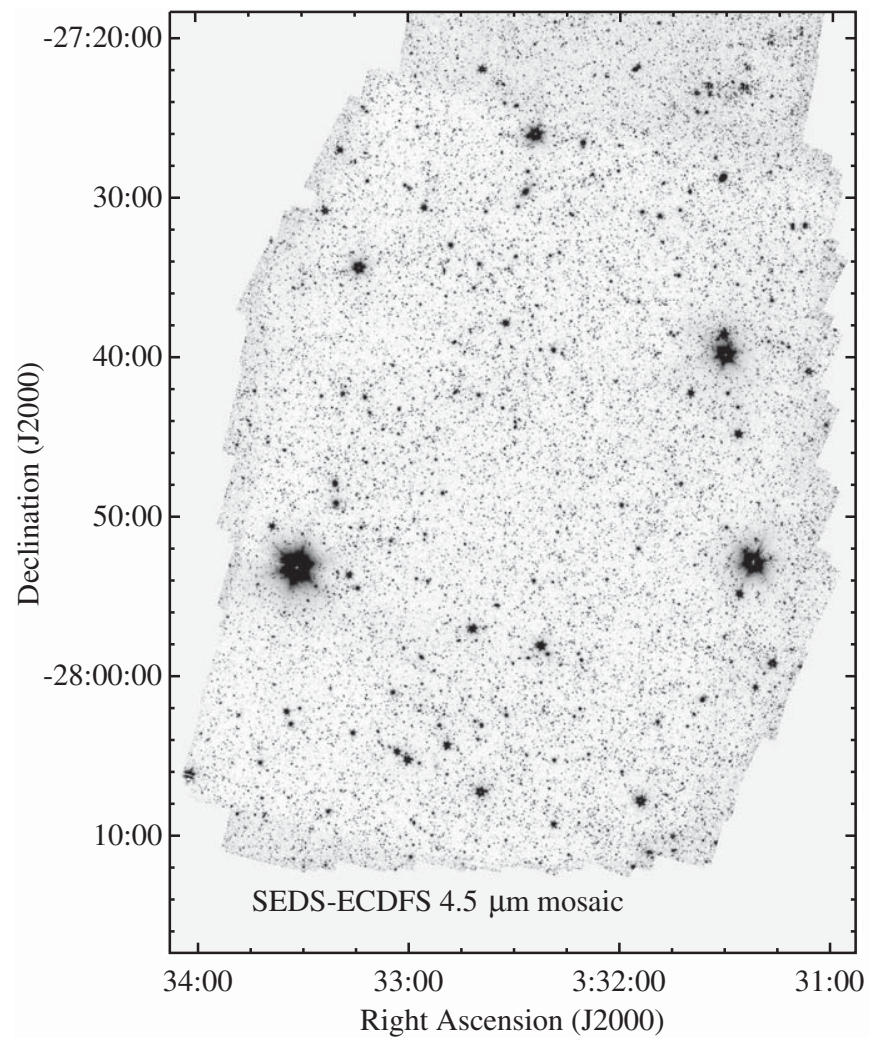

Figure 8. As Figure 7, but showing the full-depth mosaic of the ECDFS including all SEDS and cryogenic imaging by IRAC at $4.5 \mu \mathrm{m}$. The stretch ranges from 0 to $0.05 \mathrm{MJy} \mathrm{sr}^{-1}$.

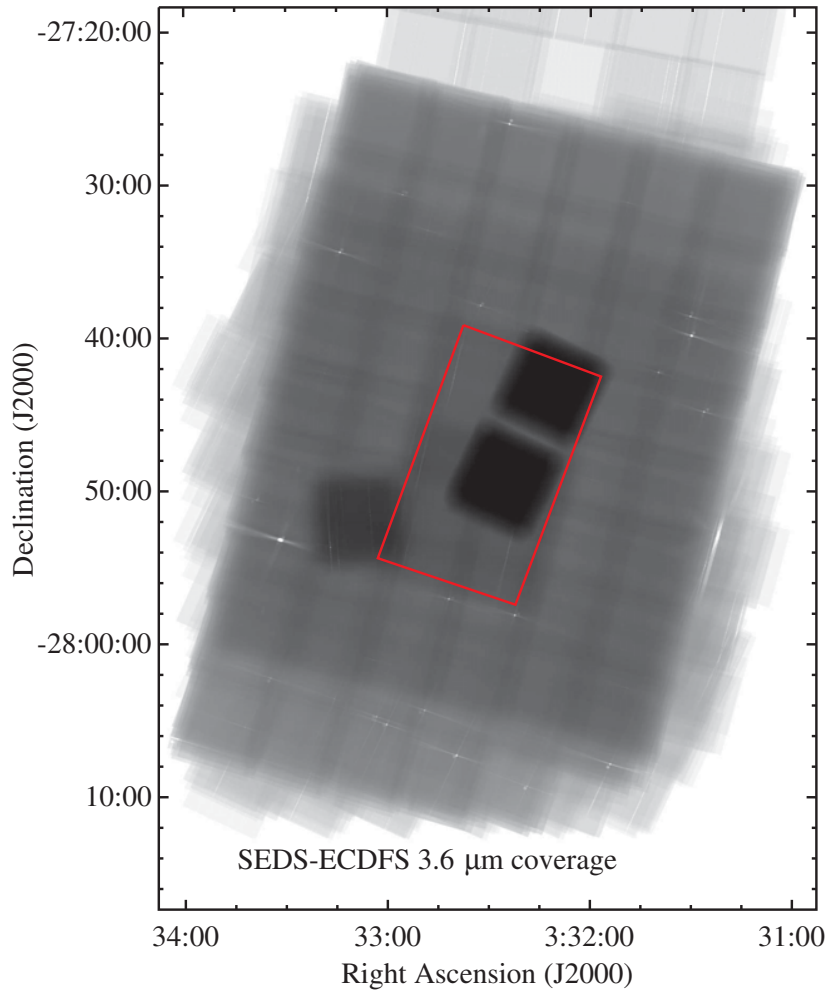

Figure 9. The total IRAC $3.6 \mu \mathrm{m}$ coverage map in the ECDFS including all data from SEDS as well as the cryogenic mission. The stretch is logarithmic and ranges from 0 (white) to $300 \mathrm{ks}$. The total area observed to at least $10 \mathrm{ks}$ depth is $0.34 \mathrm{deg}^{2}$. The area surveyed by the HST MCT project CANDELS with WFC3 and ACS is indicated in red. The deep PID 70204 imaging appears as adjacent, very dark squares inside the CANDELS field. The deepest IRAC integration is well over $100 \mathrm{hr}$.

(A color version of this figure is available in the online journal.)

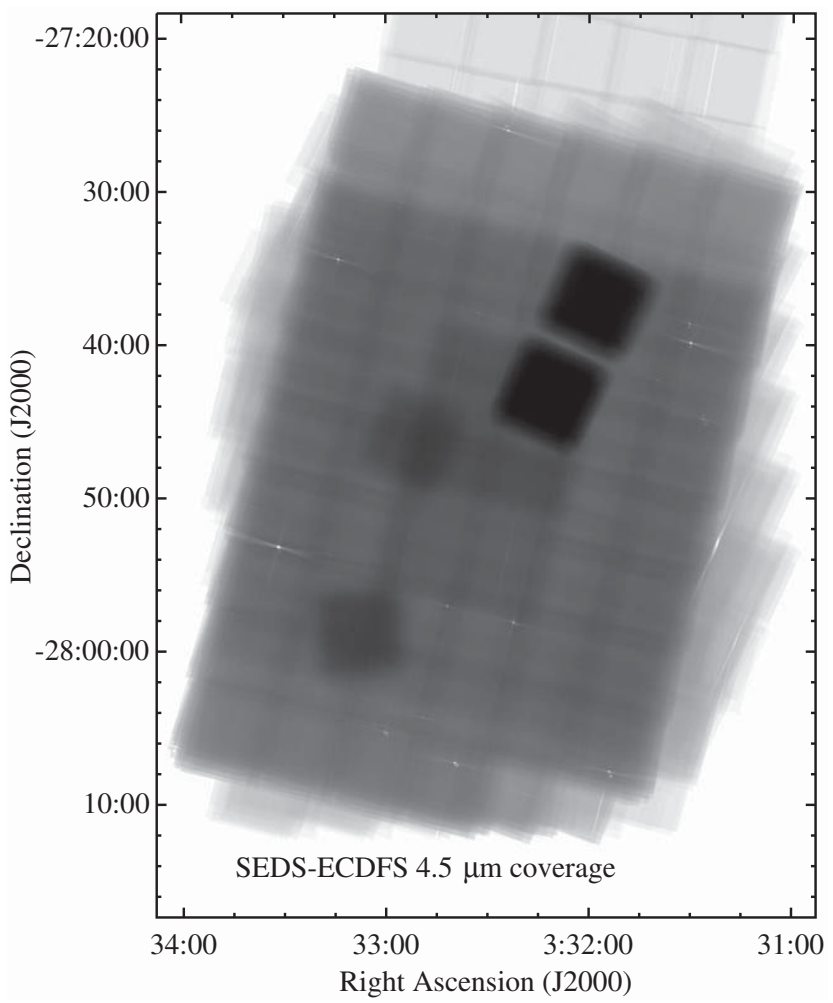

Figure 10. As Figure 9, but showing the full-depth IRAC $4.5 \mu \mathrm{m}$ coverage map in the ECDFS. The stretch is logarithmic and ranges from 0 (white) to $300 \mathrm{ks}$. The total area observed to at least $10 \mathrm{ks}$ depth is $0.34 \mathrm{deg}^{2}$. 


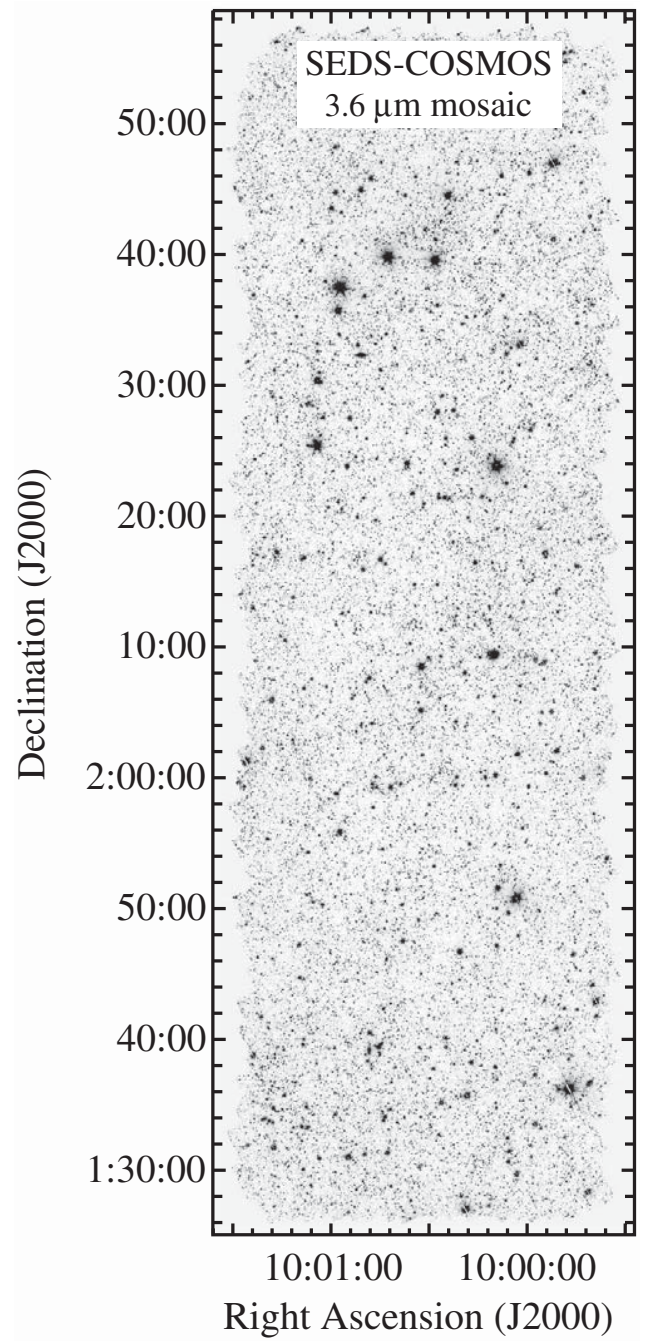

Figure 11. The total IRAC $3.6 \mu \mathrm{m}$ mosaic in the COSMOS field including all observations from SEDS and the cryogenic mission (Table 1). The image stretch ranges from -0.002 (white) to $0.05 \mathrm{MJy} \mathrm{sr}^{-1}$ (black).

epochs 1 and 3, but the coverage for epoch 2 differed because of the offset placement of the two IRAC detectors within the Spitzer focal plane and the constraints imposed by the spacecraft roll angle.

Each SEDS epoch accumulated $4 \mathrm{hr}$ integration time per pointing or less when there was pre-existing coverage of a field. Each Astronomical Observation Request (AOR), representing a continuous sequence of Spitzer observations, looked at each pointing position exactly once and covered an entire field. ${ }^{33}$ For efficiency, each position in the field was observed for 2-4 frames of $100 \mathrm{~s}$ each before moving the telescope to the next position. There was no dithering built into individual AORs, but AOR origins were offset by $\sim 1^{\prime}$ in a Rouleaux pattern. Thus each field required 36-72 observations at each pointing (minus pre-existing coverage), and these were spread over the nearly the entire range of each epoch. Therefore each field was sampled with near-uniform time coverage and point-spread function (PSF). Individual AORs had different spacings between pointings and pointings observed in different time orders. The AORs thus sampled each sky position at a variety of positions on the arrays and also ensured that those positions included

\footnotetext{
33 In some cases, grouped AORs were needed instead of a single one, but the
} effect was still to cover the entire field in a time interval of less than a day.

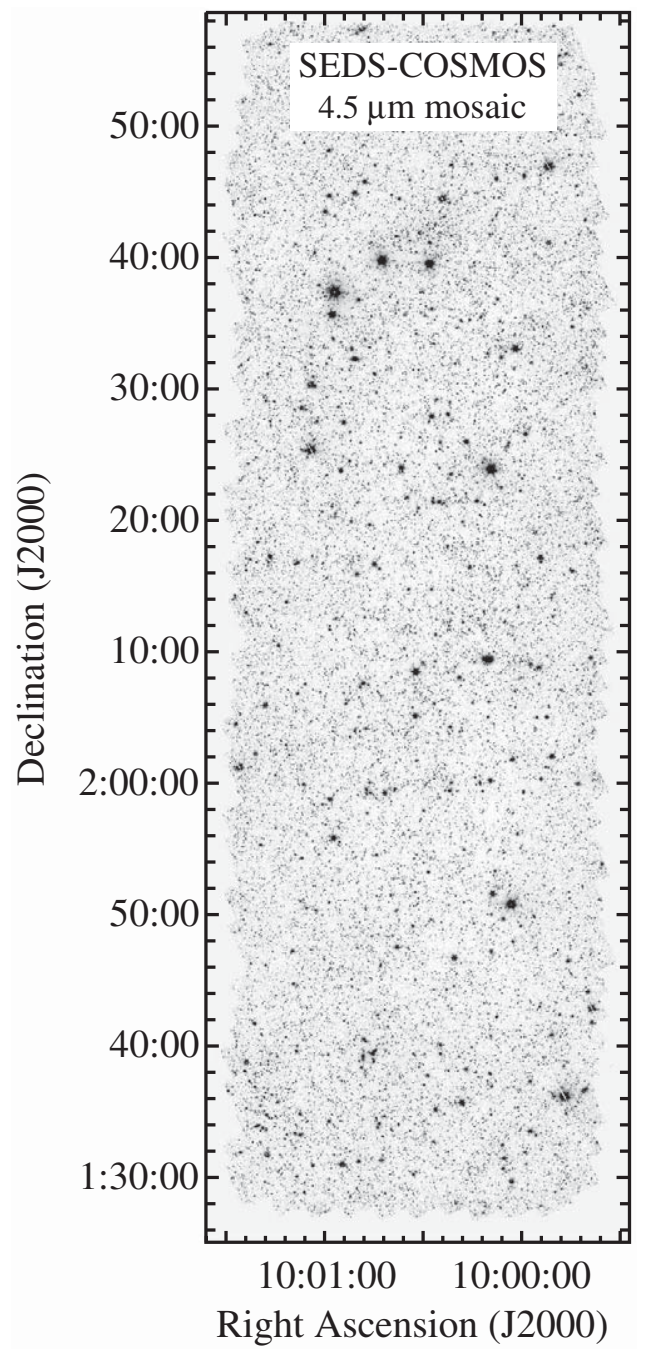

Figure 12. As Figure 11, but showing the full-depth $4.5 \mu \mathrm{m}$ mosaic in the COSMOS field. The stretch ranges from -0.002 to $0.05 \mathrm{MJy} \mathrm{sr}^{-1}$.

both near and far spacings. These characteristics minimize data artifacts.

Two of the fields, EGS and COSMOS, are long and narrow $\left(\sim 1^{\circ} \times 10^{\prime}\right)$. The map pattern for these was two IRAC FOVs (each $5^{\prime}$ ) in width by a variable number of FOVs in length. Visibility constraints prevented the COSMOS strip from aligning with the IRAC arrays, and the width mapped to full depth in both FOVs was $\sim 8^{\prime}$. The other fields (UDS, HDFN, ECDFS) are approximately square, and the basic map grid was rectangular but with varying spacings. A few positions in the centers of the HDFN and ECDFS fields already possessed deep coverage ( $223 \mathrm{hr}$ ), and pointings that would duplicate this coverage (four in ECDFS, two in HDFN) were omitted. The UDS field has bright stars in its northeast corner, and coverage was designed to exclude these stars.

\subsection{Data Reduction}

To the maximum extent possible, identical reduction procedures were applied to all SEDS data so as to facilitate subsequent variability studies and to ensure a uniform data quality throughout the SEDS project. The data reduction was based on version S18.8.0 of the IRAC Corrected Basic Calibrated Data (cBCD) exposures. All 3.6 and $4.5 \mu \mathrm{m}$ cBCD frames were object-masked and median-stacked on a per-AOR basis; the 


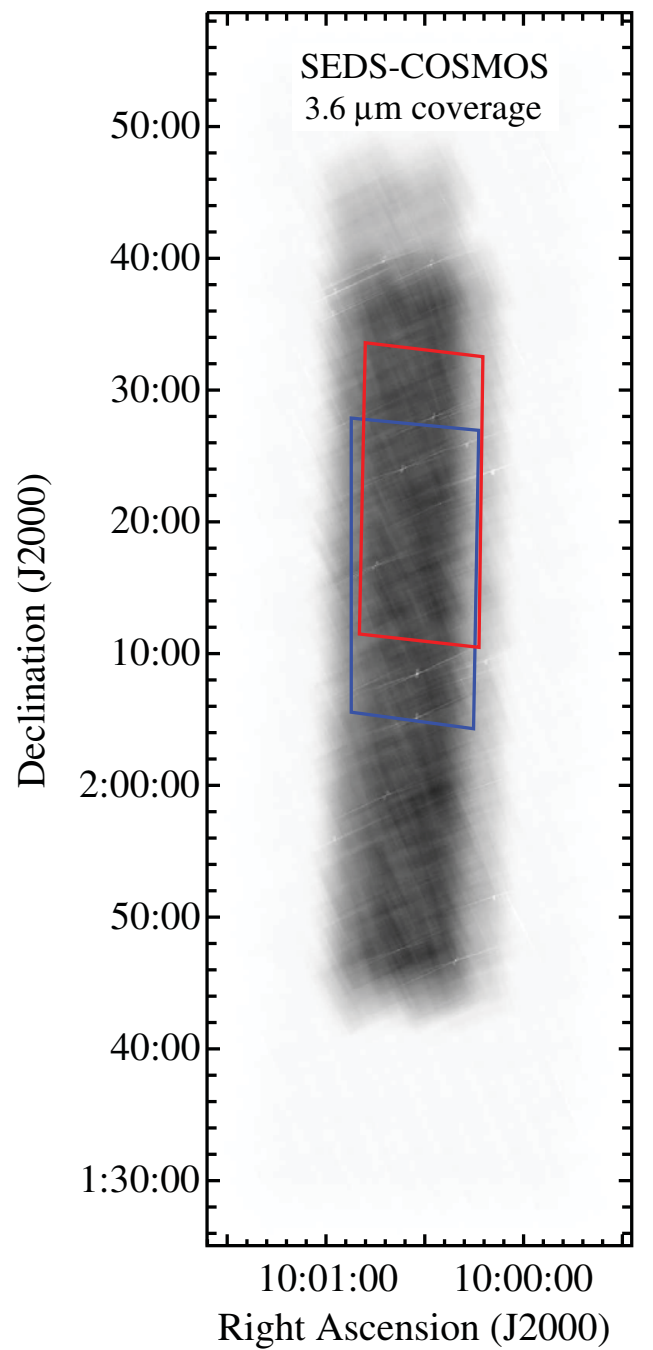

Figure 13. The total SEDS $3.6 \mu \mathrm{m}$ coverage map of COSMOS corresponding to the mosaic shown in Figure 11. The linear stretch ranges from 0 (white) to $70 \mathrm{ks}$ (black). The deepest coverage is $58.4 \mathrm{ks}$. The total area observed with at least $10 \mathrm{ks}$ depth is $0.22 \mathrm{deg}^{2}$. The areas surveyed with the WFC3 and ACS instruments by CANDELS are outlined respectively in red and blue.

(A color version of this figure is available in the online journal.)

resulting stacked images (presumed to represent blank sky) were visually inspected and subtracted from individual cBCDs within each AOR to eliminate long-term residual images arising from prior observations of bright sources. The sky-subtracted cBCDs were then examined individually and processed using custom software routines to correct column-pulldown effects associated with bright sources. The code, known as the "WarmMission Column Pulldown Corrector," is publicly available at the Spitzer Science Center. ${ }^{34}$

After these preliminaries, the SEDS exposures and the coincident IRAC imaging from earlier programs (Section 2) were mosaiced together into 3.6 and $4.5 \mu \mathrm{m}$ mosaics using IRACproc (Schuster et al. 2006). IRACproc implements the standard IRAC reduction software (MOPEX). The standard MOPEX error propagation techniques, which are based on the IRAC uncertainty images, were employed by IRACproc, with one significant exception. IRACproc augments the capabilities of MOPEX by calculating the spatial derivative of each image and adjusting the clipping algorithm accordingly. Pixels where the derivative

\footnotetext{
34 http://ssc.spitzer.caltech.edu/archanaly/contributed/browse.html
}

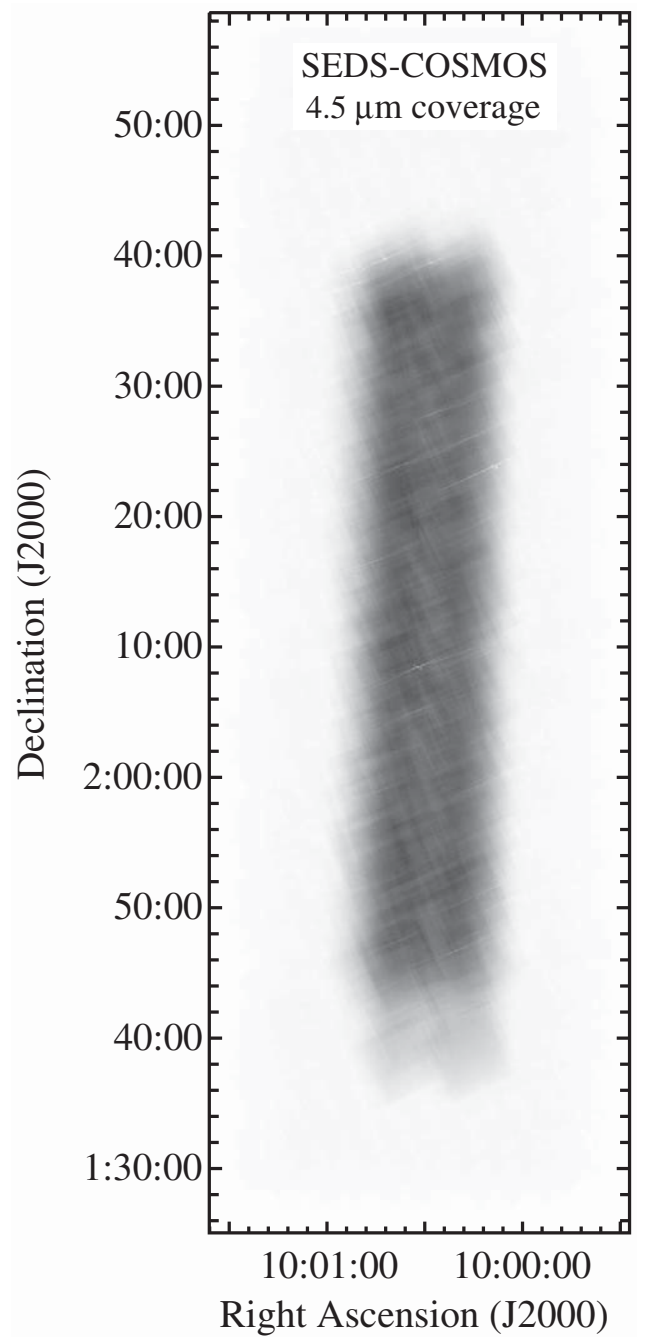

Figure 14. As Figure 13, but showing the full-depth $4.5 \mu \mathrm{m}$ coverage map of the COSMOS field. The linear stretch ranges from 0 (white) to 70 ks (black); the deepest coverage in this field is $55 \mathrm{ks}$. The total area observed with at least $10 \mathrm{ks}$ depth is $0.21 \mathrm{deg}^{2}$.

is low (in the field) are clipped more aggressively than are pixels where the spatial derivative is high (point sources). This avoids downward biasing of point source fluxes in the output mosaics that might otherwise occur because of the slightly undersampled IRAC PSF. The software was configured to automatically flag and reject cosmic ray hits based on pipeline-generated masks together with the adjusted sigma-clipping algorithm for spatially coincident pixels.

In order to take advantage of the subpixel shifts of our mapping strategy, and to facilitate registration of the IRAC mosaics to the coextensive CANDELS imaging (0'06 pixels; Grogin et al. 2011; Koekemoer et al. 2011), the mosaics were resampled to 0!'6 pixel $^{-1}$. Thus each SEDS mosaic pixel subtends approximately one-fourth the area of the native IRAC pixel. The final mosaics' tangent-plane projections were likewise aligned to those used by the CANDELS mosaics.

The 40 final mosaics and coverage maps (including three epochs and a total coadd in each of the two IRAC bands for each of the five fields) are all available from the SEDS team Web site. ${ }^{35}$

\footnotetext{
35 http://www.cfa.harvard.edu/SEDS
} 


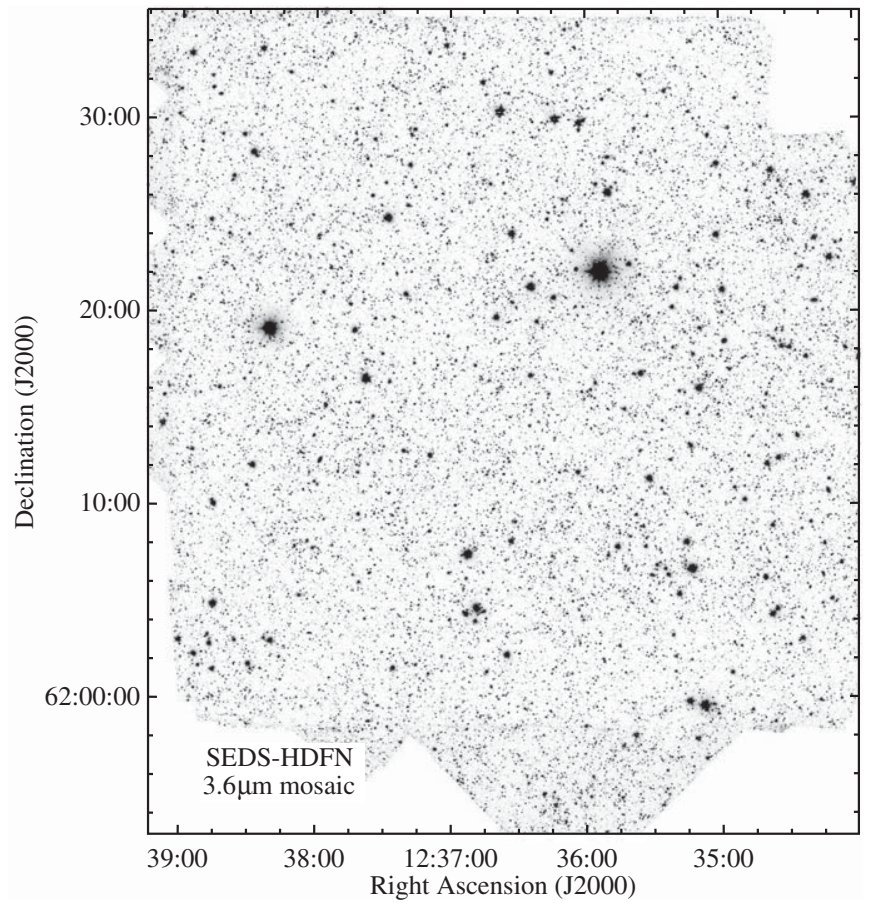

Figure 15. Total SEDS IRAC $3.6 \mu \mathrm{m}$ mosaic in the HDFN field, with the observations from the warm mission included. The stretch ranges from 0 to $0.05 \mathrm{MJy} \mathrm{sr}^{-1}$.

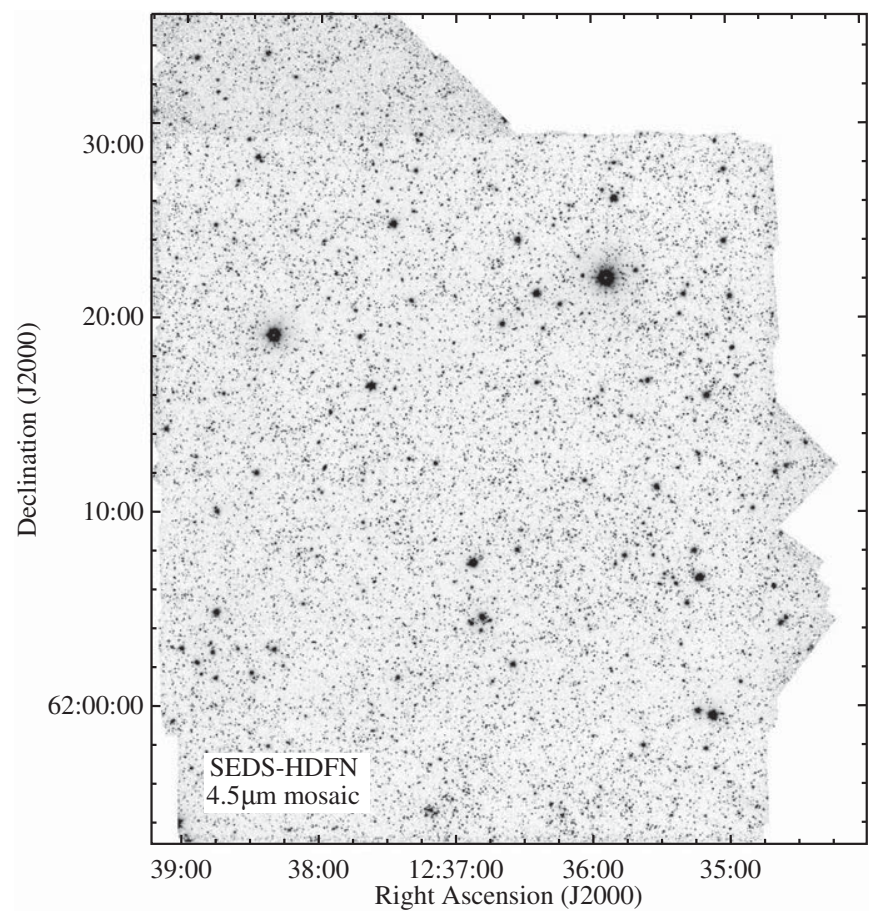

Figure 16. As Figure 15, but for the $4.5 \mu \mathrm{m}$ mosaic.

\section{SOURCE EXTRACTION AND PHOTOMETRY}

\subsection{Source Identification}

A first test of source extraction methods was made in the SEDS EGS field with SExtractor (ver. 2.5.0; Bertin \& Arnouts 1996), a standard tool for these purposes (e.g., Lonsdale et al. 2003; Ashby et al. 2009). The software was configured identically to that used by Barmby et al. (2008) to photometer sources in their (shallower) EGS mosaic. Despite the fact that

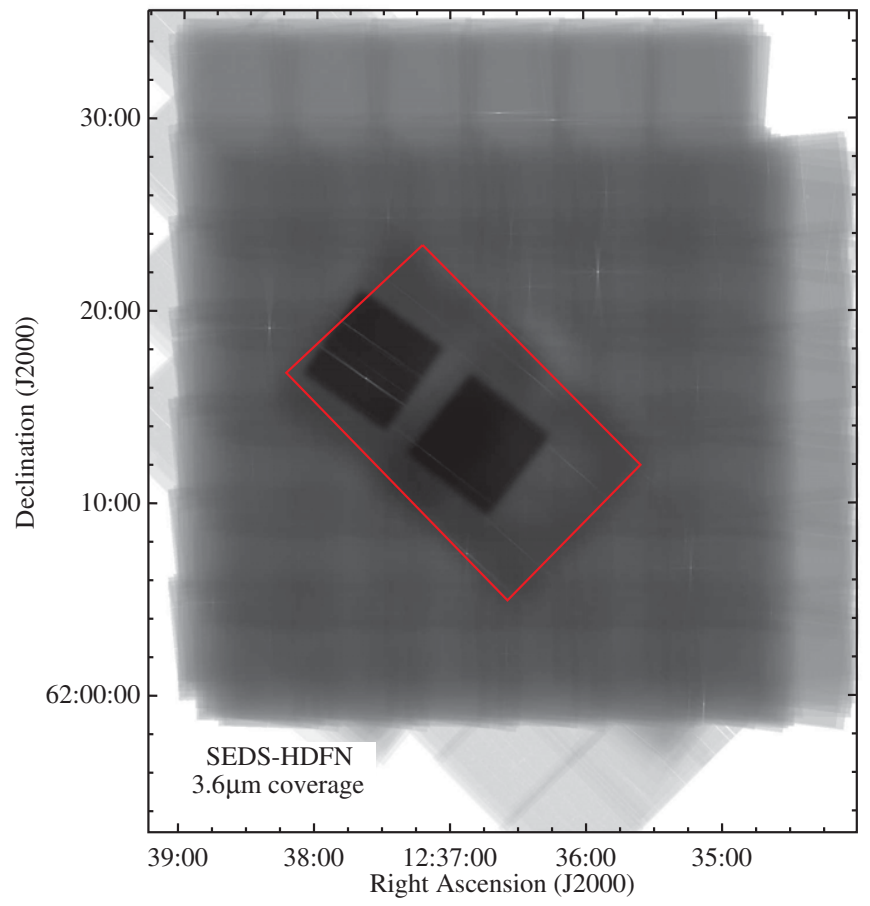

Figure 17. Total SEDS IRAC $3.6 \mu \mathrm{m}$ coverage map in the HDFN field. The logarithmic stretch ranges from 0 (white) to 2000; the deepest coverage in this field is roughly $160 \mathrm{ks}$. The red rectangle indicates approximately the area covered by the CANDELS observations. The total area observed with at least $10 \mathrm{ks}$ is $0.30 \mathrm{deg}^{2}$.

(A color version of this figure is available in the online journal.)

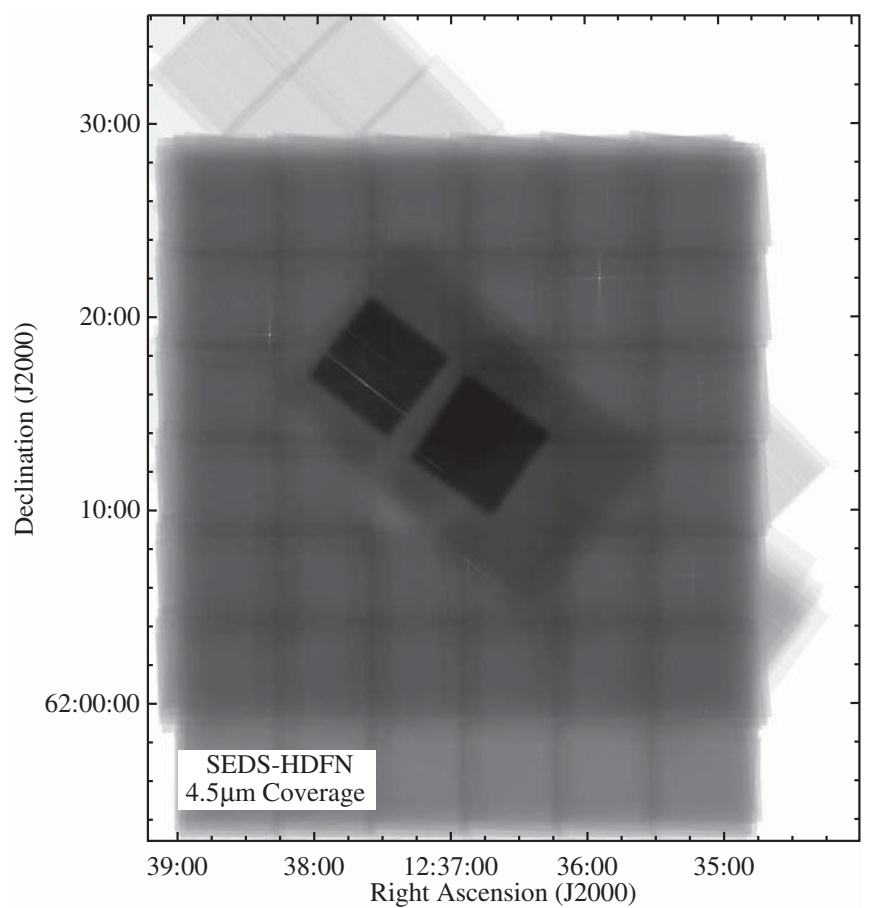

Figure 18. As Figure 17, but for the SEDS $4.5 \mu \mathrm{m}$ coverage. The total area observed with at least $10 \mathrm{ks}$ is $0.28 \mathrm{deg}^{2}$.

the SEDS EGS mosaics incorporate a factor of four longer integration time per pixel, the resulting SExtractor catalogs improved only marginally upon those of Barmby et al. In effect, source confusion imposed a limit on our ability to identify faint sources that SExtractor could not overcome. This behavior has been remarked on before (e.g., Sanders et al. 2007). We therefore 


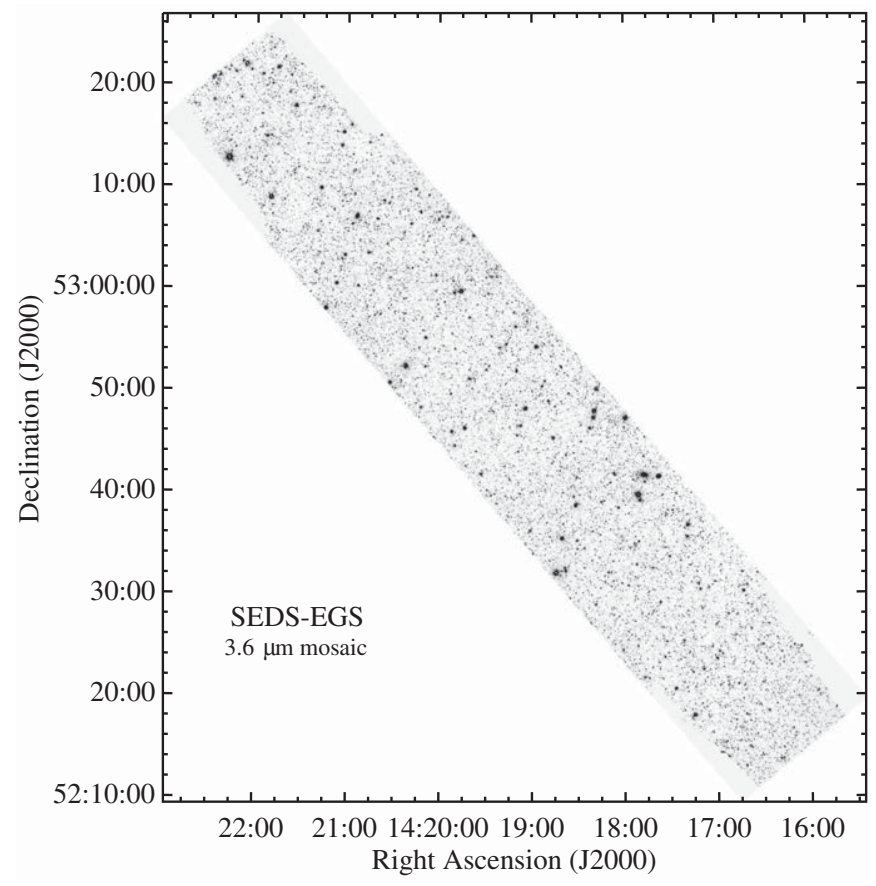

Figure 19. Total SEDS IRAC $3.6 \mu \mathrm{m}$ mosaic in the EGS field. The stretch ranges from 0 to $0.05 \mathrm{MJy} \mathrm{sr}^{-1}$.

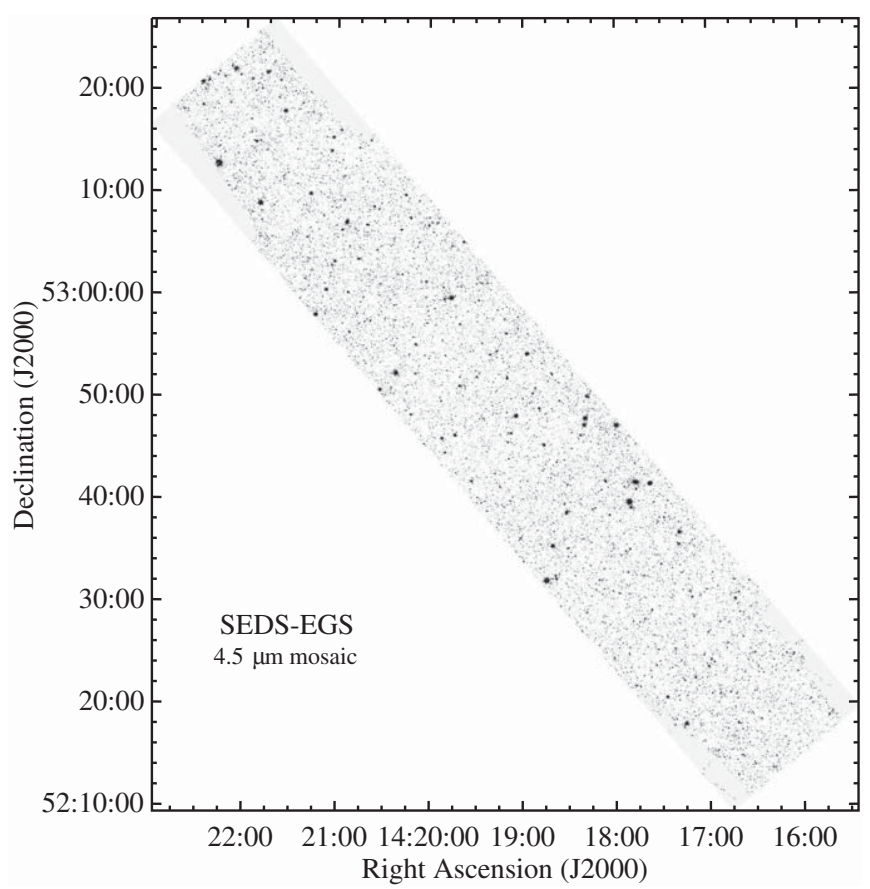

Figure 20. Total SEDS IRAC $4.5 \mu \mathrm{m}$ mosaic in the EGS field. The stretch ranges from 0 to $0.05 \mathrm{MJy} \mathrm{sr}^{-1}$.

used StarFinder (version 1.6f; Diolaiti et al. 2000) to identify IRAC sources in the SEDS fields. StarFinder is optimized for identification of blended sources in crowded wide-field adaptive-optics observations. StarFinder works by repeatedly fitting and subtracting a PSF to sources it identifies in an image. It begins with the brightest object. After the brightest source has been identified, fitted, and subtracted, the next brightest object is treated in the resulting residual image. StarFinder iterates

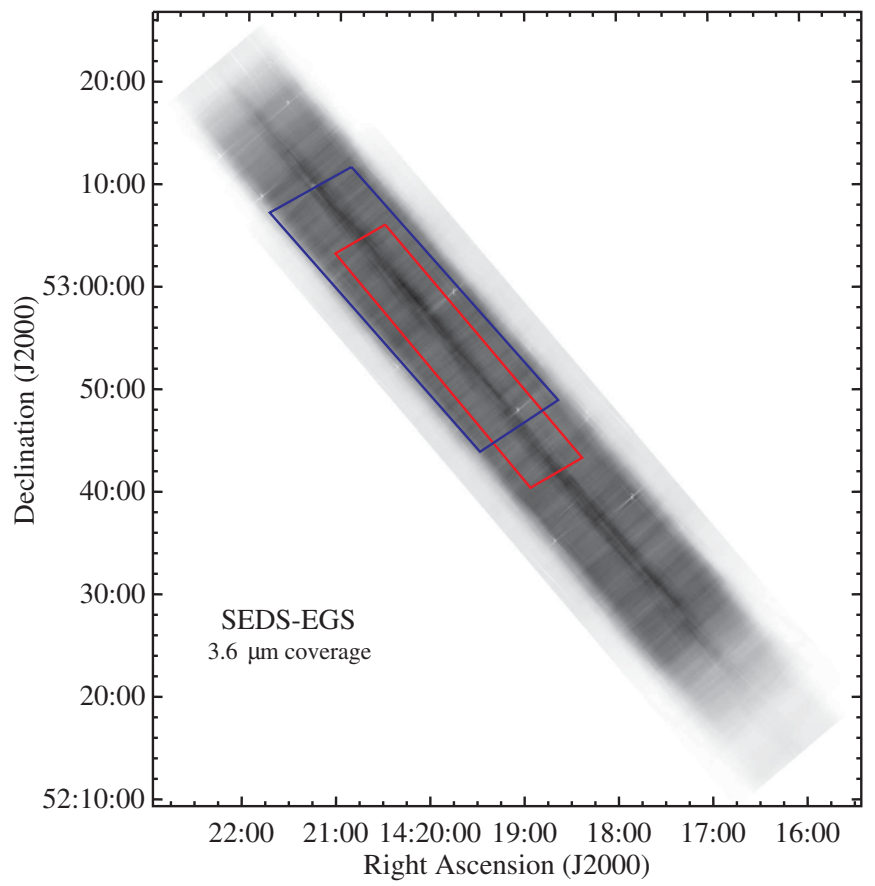

Figure 21. Total SEDS IRAC $3.6 \mu \mathrm{m}$ coverage map in the EGS field. The linear stretch ranges from 0 (white) to 700; the deepest effective coverage shown is roughly $20.3 \mathrm{hr}$. The total area observed with at least $10 \mathrm{ks}$ is $0.26 \mathrm{deg}^{2}$. The areas surveyed with the WFC 3 and ACS instruments by CANDELS are outlined respectively in red and blue.

(A color version of this figure is available in the online journal.)

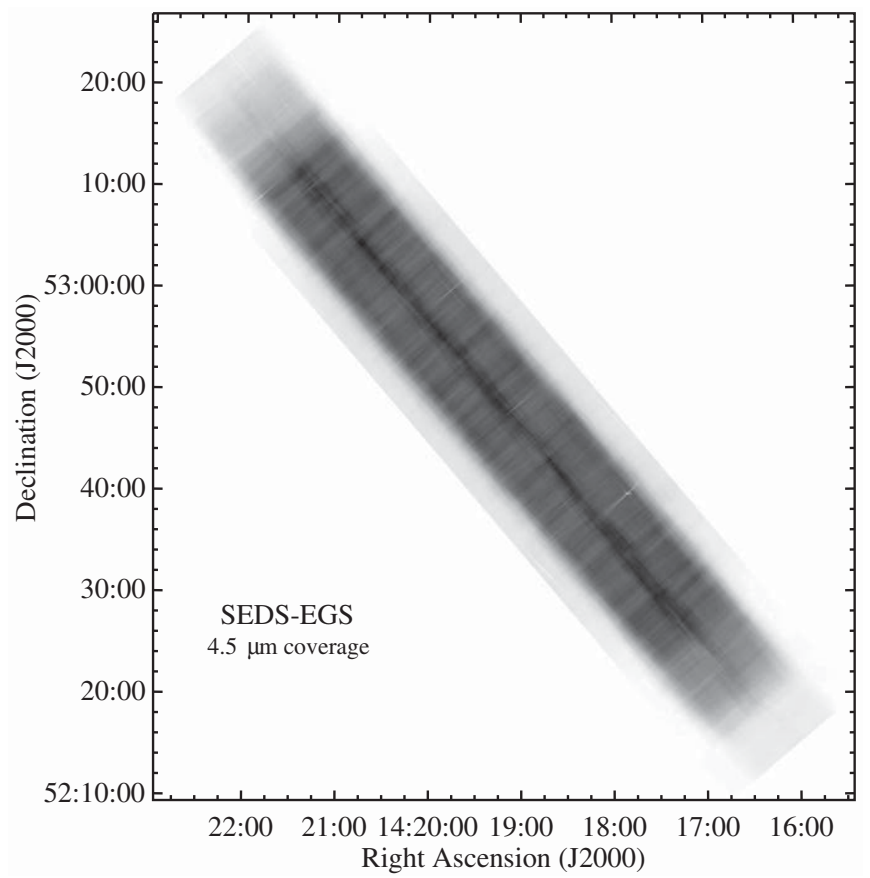

Figure 22. Total SEDS IRAC $3.6 \mu \mathrm{m}$ coverage map in the EGS field. The linear stretch ranges from 0 (white) to 700 ; the deepest effective coverage shown is roughly $21.1 \mathrm{hr}$. The total area observed with at least $10 \mathrm{ks}$ is $0.25 \mathrm{deg}^{2}$.

this process, operating on progressively fainter sources, until a user-specified limiting sensitivity is reached.

The SEDS catalogs were constructed in two steps. First, StarFinder was used to locate sources (even faint, blended ones). Second, a custom code was used to correct biases in the StarFinder photometry. 
Table 2

SEDS Aperture Corrections

\begin{tabular}{|c|c|c|c|c|c|c|}
\hline \multirow[t]{2}{*}{ Field } & \multicolumn{6}{|c|}{ Aperture Diameters (arcseconds) } \\
\hline & 2.4 & 3.6 & 4.8 & 6.0 & 7.2 & 12.0 \\
\hline \multicolumn{7}{|c|}{$3.6 \mu \mathrm{m}$} \\
\hline UDS & 2.29 & 1.52 & 1.29 & 1.20 & 1.16 & 1.08 \\
\hline ECDFS & 2.16 & 1.50 & 1.28 & 1.17 & 1.14 & 1.07 \\
\hline COSMOS & 2.11 & 1.45 & 1.25 & 1.16 & 1.13 & 1.06 \\
\hline HDFN & 2.28 & 1.51 & 1.28 & 1.19 & 1.15 & 1.07 \\
\hline EGS & 2.26 & 1.54 & 1.30 & 1.19 & 1.16 & 1.08 \\
\hline \multicolumn{7}{|c|}{$4.5 \mu \mathrm{m}$} \\
\hline UDS & 2.27 & 1.57 & 1.33 & 1.21 & 1.18 & 1.10 \\
\hline ECDFS & 2.19 & 1.49 & 1.27 & 1.18 & 1.15 & 1.07 \\
\hline COSMOS & 2.15 & 1.50 & 1.27 & 1.17 & 1.14 & 1.07 \\
\hline HDFN & 2.22 & 1.52 & 1.29 & 1.18 & 1.14 & 1.08 \\
\hline EGS & 2.24 & 1.50 & 1.28 & 1.19 & 1.16 & 1.08 \\
\hline
\end{tabular}

Notes. Aperture corrections (multiplicative factors) derived from empirical PSFs constructed using at least 10 stars in each of the SEDS fields. The corrections are defined so that when applied, they convert SEDS aperture photometry to total 24" aperture diameter magnitudes, as used for Spitzer calibration stars. All photometry in the SEDS catalogs presented in this work has been aperturecorrected using these values.

The source-location step used the full-depth SEDS mosaics masked to exclude regions of shallow coverage. This exclusion of relatively noisy low-coverage regions-virtually all located on the periphery of the SEDS footprints-was done to prevent them from artificially inflating the rms-threshold criteria needed to search deeply for faint objects in the areas of highest signal/ noise. The 3.6 and $4.5 \mu \mathrm{m}$ mosaics were masked separately, i.e., the coverage was not initially required to be coextensive.

The source-location process began with the creation of empirical PSFs for the SEDS fields. Isolated, unsaturated stars (between magnitudes 10 and 15, depending on the field and the coverage) were inspected (to exclude binaries) and then scaled to a common, fiducial intensity. Objects within 64 mosaic pixels (38."4) of each PSF star were masked, and the resulting scaled images were median stacked to suppress artifacts. The halos of the resulting composite, high-dynamic-range PSFs were then smoothed slightly at large radii to suppress noise. They were also masked at radii beyond 64 pixels. Because the scale factors applied prior to the median stacking can be large for relatively faint stars, the scaling can lead to spurious negativevalued features that create obvious artifacts in the residual images described below. We therefore masked negative-valued pixels in the composite PSFs to zero. All the PSFs were then normalized to unity integrated flux, per standard StarFinder protocol. Correction factors were then estimated by measuring the flux from each PSF star within a series of apertures covering a range of diameters (Table 2).

With the cleaned and normalized PSFs, iterative source identification was carried out as described above on the masked mosaics. To prevent bright sources from distorting the estimates of backgrounds nearby, StarFinder was configured to estimate the backgrounds with a grid spacing equal to 72 times the FWHM of the PSF. It was also set to estimate backgrounds under detected sources. A spacing constraint was imposed, requiring that sources be separated by at least the half of the PSF width (defined as the half-width at half-maximum or HWHM; 0!9) in order to be counted as separate detections; blended sources lying closer to each other were counted as a single object.
StarFinder computes the rms of the source-free pixels to estimate the significance of source detections. In the first StarFinder run, sources were counted as detections if they exceeded $5 \sigma$ significance relative to the estimated rms variations. After the first run had removed the $5 \sigma$ sources, StarFinder was run a second time but with a $3 \sigma$ threshold on the residual images, i.e., a new (lower) rms was computed and then applied to science mosaics from which all previously-detected sources had been removed. Finally, StarFinder was run a third time on the sourcesubtracted residual from the second iteration, again with a $3 \sigma$ detection criterion. Figure 23 shows StarFinder residual images for a typical small subregion (in this case, within the EGS). Although bright and extended sources have significant residuals, those for the more abundant faint sources are sufficiently small that the residual image is far from being confused in either band. The SEDS catalogs consist of the source positions and magnitudes determined in all three StarFinder runs, modulo the photometric corrections described below.

\subsubsection{Photometric Corrections}

StarFinder is very efficient for identifying sources, but it measures only PSF-fitted photometry. While this is perfectly acceptable for some sources (Milky Way stars, QSOs), it is inadequate for galaxies because they are seldom point sources. It was therefore necessary to convert the PSF-fitted photometry to aperture photometry in order to measure total fluxes for galaxies.

To generate the SEDS aperture photometry we used a custom code that operated on the StarFinder catalogs and residual images. The code first re-created the original appearance of each source in turn by re-inserting the scaled/fitted PSF at the position of that object, effectively adding in the fitted flux to any residuals that remained. Thus each source was re-created at its original position with its original flux, with the PSF fits to all other sources removed. The code then re-measured the flux of this source in a suite of apertures (Table 2). Backgrounds were estimated within annuli centered on the sources but outside the photometric apertures. The procedure was repeated for all detected sources. This approach had two significant advantages. First, it greatly improved the background estimates because to a good approximation all neighboring sources had been removed. Second, it accounted for the fact that galaxies are not generally point sources; aperture photometry requires no assumptions about the surface brightness distributions.

The multiaperture photometry was corrected to total magnitudes using the aperture corrections measured separately for the empirical PSFs in each band and field. All SEDS catalog entries include these aperture corrections, which are given in Table 2 and Figure 24.

\subsection{Completeness and Depth Simulations}

SEDS survey completeness and reliability were assessed with the standard Monte Carlo approach, using photometry of artificial sources. To constrain the properties of the required artificial sources for SEDS, we used a 0'.5 search radius to match the SEDS COSMOS catalog to the coextensive F160W catalog made available by the CANDELS team (Grogin et al. 2011; Koekemoer et al. 2011). Figure 25 shows the measured widths (major axes only) of the CANDELS counterparts in the F160W filter. The great majority of SEDS IRAC-detected objects are significantly smaller than the IRAC PSF; they are in effect point sources at $3.6 \mu \mathrm{m}$. At the magnitudes of interest to SEDS, i.e., objects fainter than $23 \mathrm{AB}$ mag, virtually all the IRAC detections 


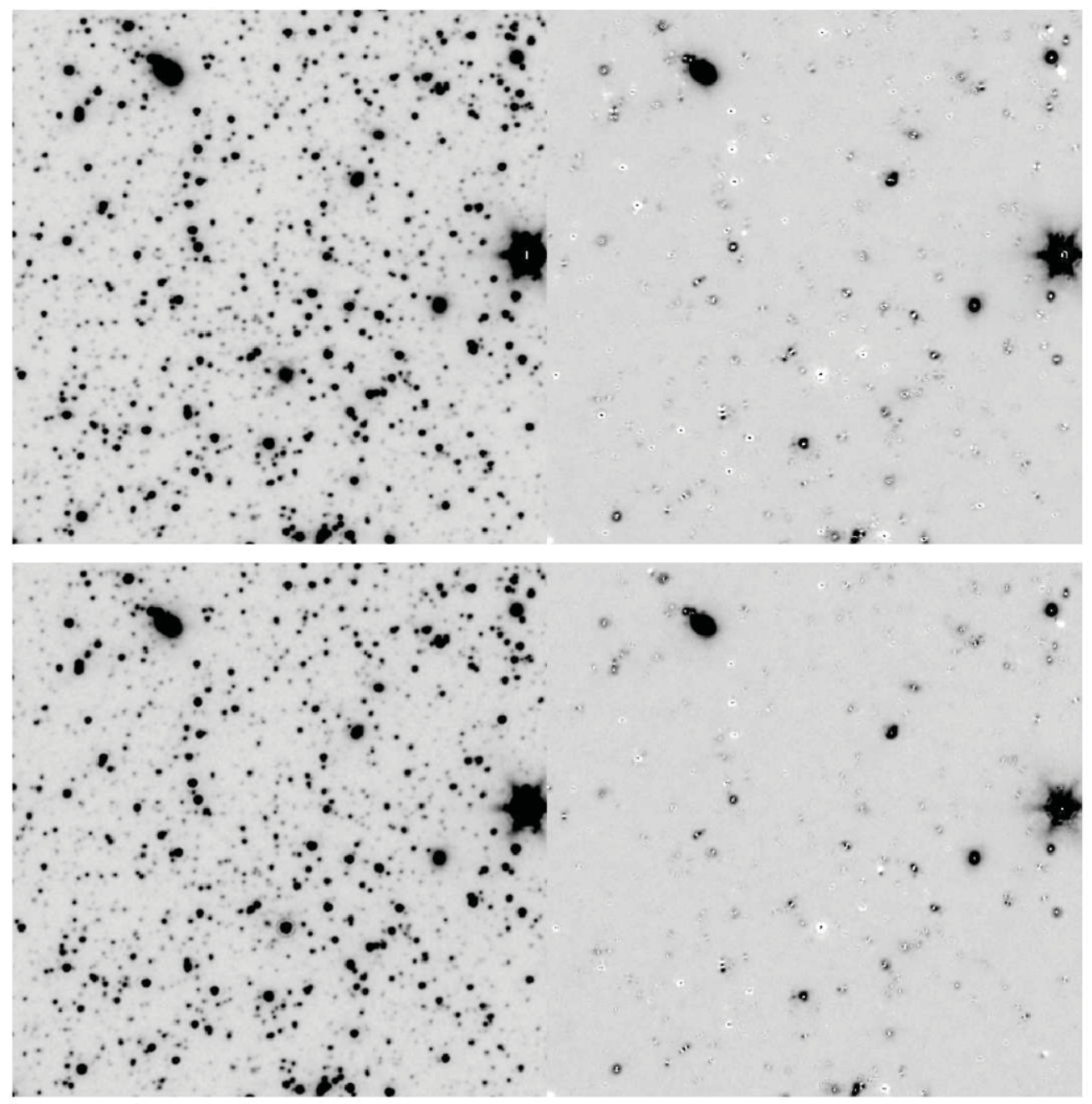

Figure 23. StarFinder fits and subtracts sources from the crowded SEDS mosaics (left) to create residual images (right) that are relatively free of contaminating flux, making it possible to identify and photometer faint IRAC sources. The linear stretch ranges from $-0.01 \mathrm{MJy} \mathrm{sr}^{-1}$ (white) to $0.04 \mathrm{MJy} \mathrm{sr}^{-1}$ (black) in all four panels in order to show the details of faint sources. Upper left panel: A $5^{\prime} \times 5^{\prime}$ region in the $3.6 \mu \mathrm{m}$ EGS mosaic. Upper right panel: The same field after all StarFinder-detected sources have been scaled and subtracted. Lower left panel: The same field as it appears in the $4.5 \mu \mathrm{m}$ mosaic. Lower right panel: The $4.5 \mu \mathrm{m}$ residual image.

are point sources. We therefore used only point sources in our completeness and depth simulations.

A minimum of $10^{5}$ artificial point sources were introduced into each SEDS mosaic. The number of artificial sources inserted simultaneously, however, was kept small (less than $2 \%$ of the number of detected IRAC sources) so as not to artificially aggravate source confusion effects. Typically, only 50-250 artificial sources were introduced at a time. Approximately the same number of sources was inserted in each 0.5 mag interval between 18 and $27 \mathrm{mag}$. They were placed at random locations throughout the portions of the science mosaics having at least $10 \mathrm{ks}$ integration time to sample all variations in large-scale structure present. The modified mosaics were then photometered with StarFinder in the same way the original detections had been made.

We followed Barmby et al. (2008) and counted an artificial source as a valid detection if its measured flux was within $50 \%$ of its "true" flux and its measured position was within 1 " of its a priori known position. Completeness was thus defined as the fraction of artificial sources over a range of apparent brightnesses in bins of width 0.5 mag extending from $100 \%$ completeness (at $\sim 18 \mathrm{mag}$ ) down to zero at $26.5-27.0 \mathrm{mag}$. The uncertainties in the completeness estimates were inferred from Poisson statistics in each bin. As Figure 26 and Table 3 show, the $3.6 \mu \mathrm{m}$ SEDS catalog is $80 \%$ and $50 \%$ complete at roughly 23.5 and $24.75 \mathrm{mag}$, respectively. The corresponding $4.5 \mu \mathrm{m}$ limits are 23.8 and $24.8 \mathrm{mag}$. Thus at magnitudes where the incompleteness correction becomes large (Figure 25), the choice of point sources for the simulations is appropriate. Some field-to-field variation is apparent in both bands.

Roughly $70 \%$ of the faintest $3.6 \mu \mathrm{m}$ StarFinder detections (25.5-26.0 mag) in the COSMOS field have no F160W counterparts. The CANDELS F160W observations reached approximately 26.5 mag $(3 \sigma)$, so genuine SEDS sources with typical colors ought to have been detected by CANDELS. The comparison suggests that StarFinder generated spurious detections at faint levels. We therefore imposed a requirement that sources must be detected at both 3.6 and $4.5 \mu \mathrm{m}$ to be included in the SEDS catalogs. Formally, the requirement imposed was that the $3.6 \mu \mathrm{m}$ detections match a $4.5 \mu \mathrm{m}$ source within 0.5 . Nearly $100 \%$ of the sources brighter than $24.5 \mathrm{mag}$ at $3.6 \mu \mathrm{m}$ were detected in both SEDS bands. At levels fainter than $24.5 \mathrm{mag}$, however, the match fraction decreases with magnitude down to the SEDS survey limit. For this reason, Figure 26 and Table 3 report the separate single-band completeness measurements for sources brighter than 24.5 mag but report (identical) two-band 


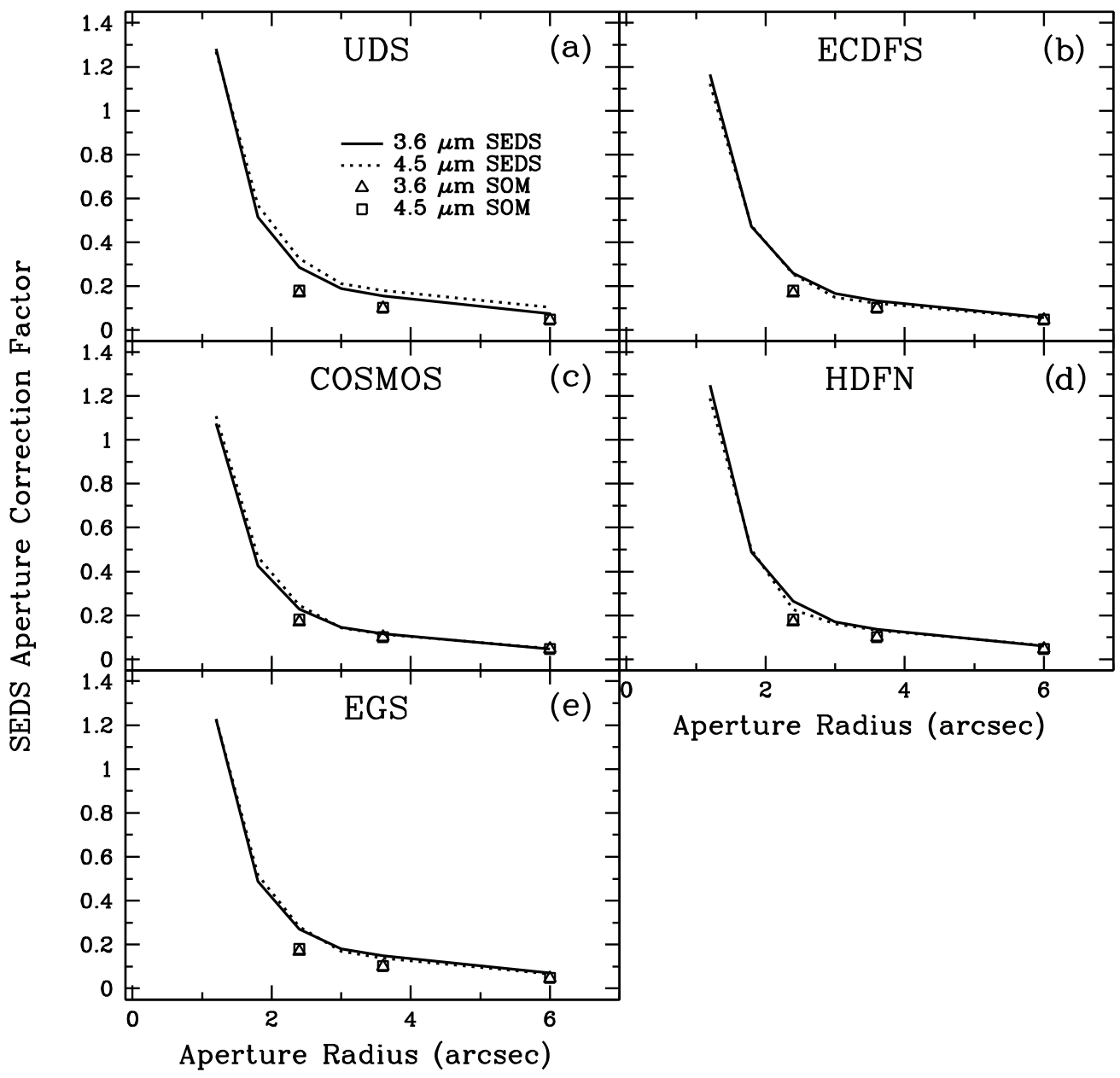

Figure 24. Aperture corrections (Table 2 factor minus one) in the five SEDS fields. Lines show empirical corrections derived from aperture photometry of the stacked PSFs of bright, unsaturated stars. Symbols represent the aperture corrections tabulated in the Spitzer Observers Manual for theoretical IRAC PSFs.

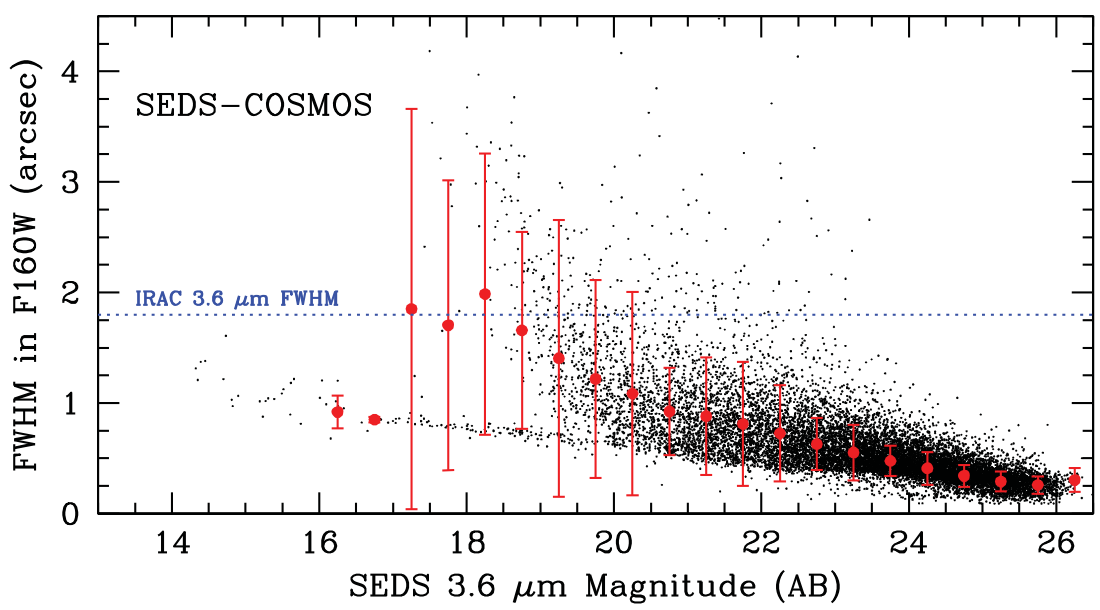

Figure 25. The angular sizes of IRAC-detected sources in the COSMOS field. Points indicate the measured widths (major axes only) of CANDELS F160W counterparts to the SEDS $3.6 \mu \mathrm{m}$ detections. The mean F160W FWHM and $1 \sigma$ uncertainties within bins of width 0.5 mag are indicated in red. Only a minority of such sources are extended compared to the FWHM of the IRAC $3.6 \mu \mathrm{m}$ PSF (indicated by the blue dotted line). All sources fainter than 23.5 AB mag are effectively point sources for SEDS.

(A color version of this figure is available in the online journal.)

completeness estimates for all SEDS sources fainter than this level.

The common practice (e.g., SExtractor) of basing photometric uncertainties on noise estimates made in source-free mosaic pixels can be problematic in some circumstances. The issue of most concern to SEDS is that the supposedly source-free pixels may well fall on faint sources lying just below the detection threshold; their fluxes may then artificially boost the uncertainty estimates. Also, sub-pixel sampling like that used for SEDS introduces correlated noise into the mosaics. 


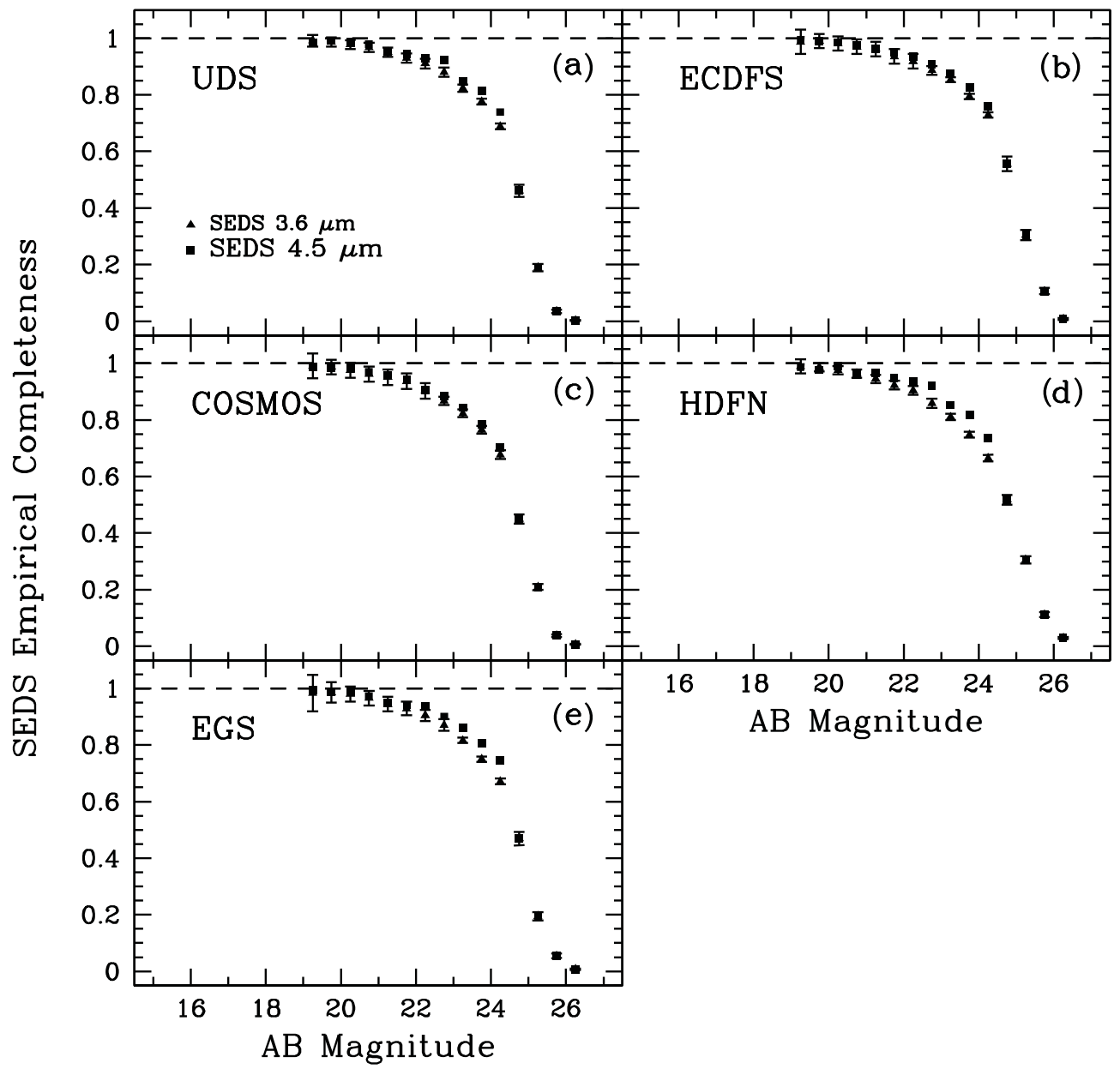

Figure 26. Completeness in the SEDS fields estimated by Monte Carlo simulations as described in Section 4.2. Solid triangles and squares correspond to the IRAC 3.6 and $4.5 \mu \mathrm{m}$ bands, respectively. The values are averages over the field areas having at least $10 \mathrm{ks}$ exposure time, but completeness in any particular small region depends on the actual integration time achieved there.

To avoid these complications the SEDS error estimates were based solely on photometry of the simulated sources with their known fluxes. We grouped the artificial sources into 0.5 mag bins and measured the offsets from the known fluxes as a function of magnitude. The standard deviations of the offsets were taken as the $1 \sigma$ measurement uncertainties and the offsets themselves were taken as the measurement biases. The results are shown in Figure 27.

The uncertainties are much more significant than the biases in all SEDS fields, at least for sources brighter than 26 mag. The uncertainty typically rises smoothly from roughly $0.03 \mathrm{mag}$ for bright sources to about 0.25 mag at $26 \mathrm{AB}$ mag. It combines contributions from three separate sources of uncertainty: (1) the $3 \%$ uncertainty in the IRAC absolute calibration (Reach et al. 2005), (2) photon noise, and (3) confusion noise. These are discussed further in Section 6.1. The empirical uncertainties are given in Table 4.

The measurement bias is relatively small for sources brighter than 20 mag. It starts to grow rapidly at roughly the level where SEDS becomes $50 \%$ incomplete and becomes greater than $0.1 \mathrm{mag}$ at $26 \mathrm{AB}$ mag. This is consistent with a picture in which faint sources are increasingly difficult to deblend from on-average brighter neighbors. The contamination of the photometric apertures by these neighbors affects the photometry, even though the measurements were made in residual images. The measurement bias is given in Table 5. The photometry reported in the SEDS catalogs has been corrected to remove this bias.

\subsection{Verification}

Because SEDS extends to extremely faint levels (by current standards) and must cope with pervasive source confusion, we verified the SEDS measurements by comparing them to previously published catalogs. We estimated the astrometric uncertainties by comparing to the USNOB and 2MASS Point Source Catalog. We verified our photometric calibration by comparing to coextensive surveys including S-COSMOS (Sanders et al. 2007), SpUDS (version DR2), EGS (Barmby et al. 2008), and SIMPLE (Damen et al. 2011).

\subsubsection{Astrometric Reliability}

To estimate the accuracy of SEDS astrometry, we compared the SEDS positions of bright but unsaturated sources to those in the Two Micron All Sky Survey (2MASS; Skrutskie et al. 2006) Point Source Catalog. We performed a search within $1^{\prime \prime}$ of the positions of IRAC sources to identify their 2MASS counterparts. The distributions of coordinate offsets for the 3.6 and $4.5 \mu \mathrm{m}$ sources are shown in Figure 28. The astrometric discrepancies are small compared to the size of a SEDS pixel: at $3.6 \mu \mathrm{m}$, the mean difference (SEDS-2MASS) was just $-0^{\prime \prime} 002 \pm 0.16$ in right ascension and $0^{\prime \prime} 004 \pm 0.19$ in declination. The corresponding mean offsets for the $4.5 \mu \mathrm{m}$ sources are similar: $-0.005 \pm 0$ !' 16 in right ascension and $0{ }^{\prime}, 03 \pm 00^{\prime} 18$ in declination. The radial uncertainties are therefore 
Table 3

Completeness in the SEDS IRAC Catalogs

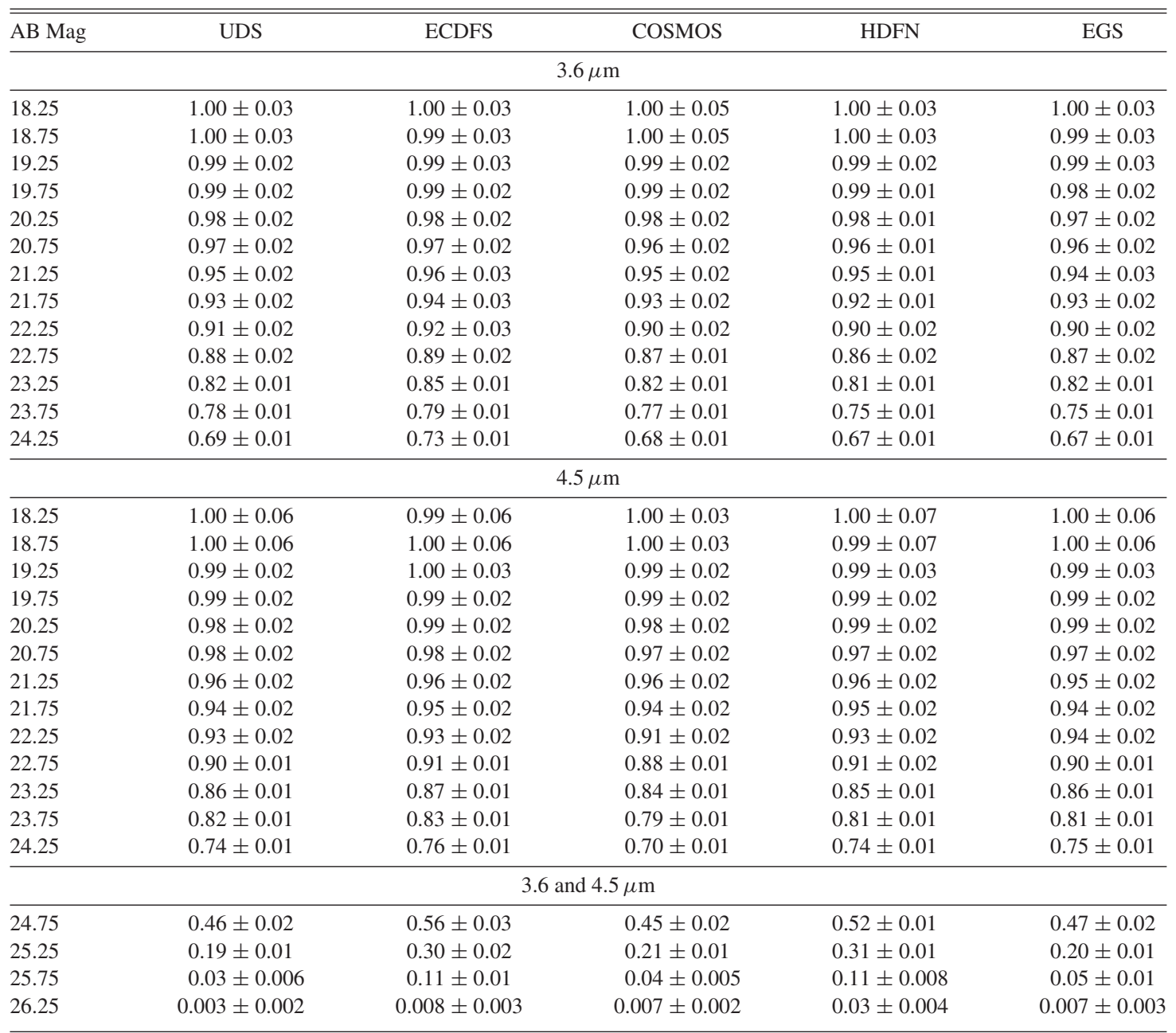

Notes. Completeness estimates for the SEDS fields. The magnitudes correspond to the centers of bins of width 0.5 mag in which the completeness was estimated. The completeness is unity at brighter magnitudes than those listed. At magnitudes $\geqslant 24.5$, completeness estimates require detection in both IRAC bands.

0 '.24 $(1 \sigma)$ relative to $2 \mathrm{MASS}$, or about 0.4 of a SEDS mosaic pixel.

We also compared the SEDS positions to their visiblewavelength counterparts in the USNOB1.0 catalog (Monet et al. 2003). We matched the positions in each of the five SEDS fields to those in USNOB1.0 using a 0.'5 search radius. Only USNOB sources having positions known to within 0.25 were matched. The mean offsets between USNOB and SEDS were insignificant for the UDS, ECDFS, and COSMOS fields. Significant offsets were found for the HDFN and EGS fields. The offsets are quantified for each field in Table 6. Users of the SEDS catalogs should be aware of and account for these offsets when matching to the USNOB positions.

\subsubsection{SEDS Photometric Verification}

To verify the SEDS photometry, we matched the SEDS cata$\operatorname{logs}$ to those of the four coextensive surveys mentioned above. In all cases, the matching was done using a 0.5 search radius, i.e., roughly twice the $1 \sigma$ astrometric uncertainty established in Section 4.3.1. Only SEDS sources brighter than the detection limits of the respective surveys were used in the comparison. For example, only sources brighter than the SpuDS faintsource detection thresholds $(2.0$ and $2.7 \mu \mathrm{Jy}$ at 3.6 and $4.5 \mu \mathrm{m}$, respectively) were matched to the SpUDS catalog. Only SEDS sources brighter than $1(1.7) \mu \mathrm{Jy}$ at 3.6 (4.5) $\mu \mathrm{m}$ were matched to S-COSMOS. The limits for the comparisons in the EGS field were set to match the $50 \%$ completeness threshold of Barmby et al. (2008), i.e., 1.5 and $1.6 \mu \mathrm{Jy}$ at 3.6 and $4.5 \mu \mathrm{m}$, respectively. Only SEDS sources brighter than 24.64 and 24.22 mag (the SIMPLE $5 \sigma$ sensitivities at 3.6 and $4.5 \mu \mathrm{m}$, respectively) were matched to the Damen et al. (2011) SIMPLE catalog. In addition, sources flagged as blended in the SIMPLE DR1 catalog were excluded from the comparison. Because all SIMPLE sources brighter than $18 \mathrm{AB}$ mag were flagged as blended, the comparison could only be carried out for objects fainter than this limit. The results are shown in Figure 29.

The SEDS photometry is broadly consistent with the previous measurements. The mean offset for faint sources varies from zero in some cases up to a maximum of $\sim 0.07$ mag for the SIMPLE $4.5 \mu \mathrm{m}$ band. The comparisons for bright SEDS sources show a somewhat different character. With the exception of the EGS $3.6 \mu \mathrm{m}$ band, SEDS sources brighter than 18 mag show a systematic offset that ranges up to a maximum of 0.1 mag in the worst case (versus SpUDS; Figures 29(a) and (b)) in the sense that these sources are brighter in the SEDS catalog on average. This may be due to the different source types that predominate on 


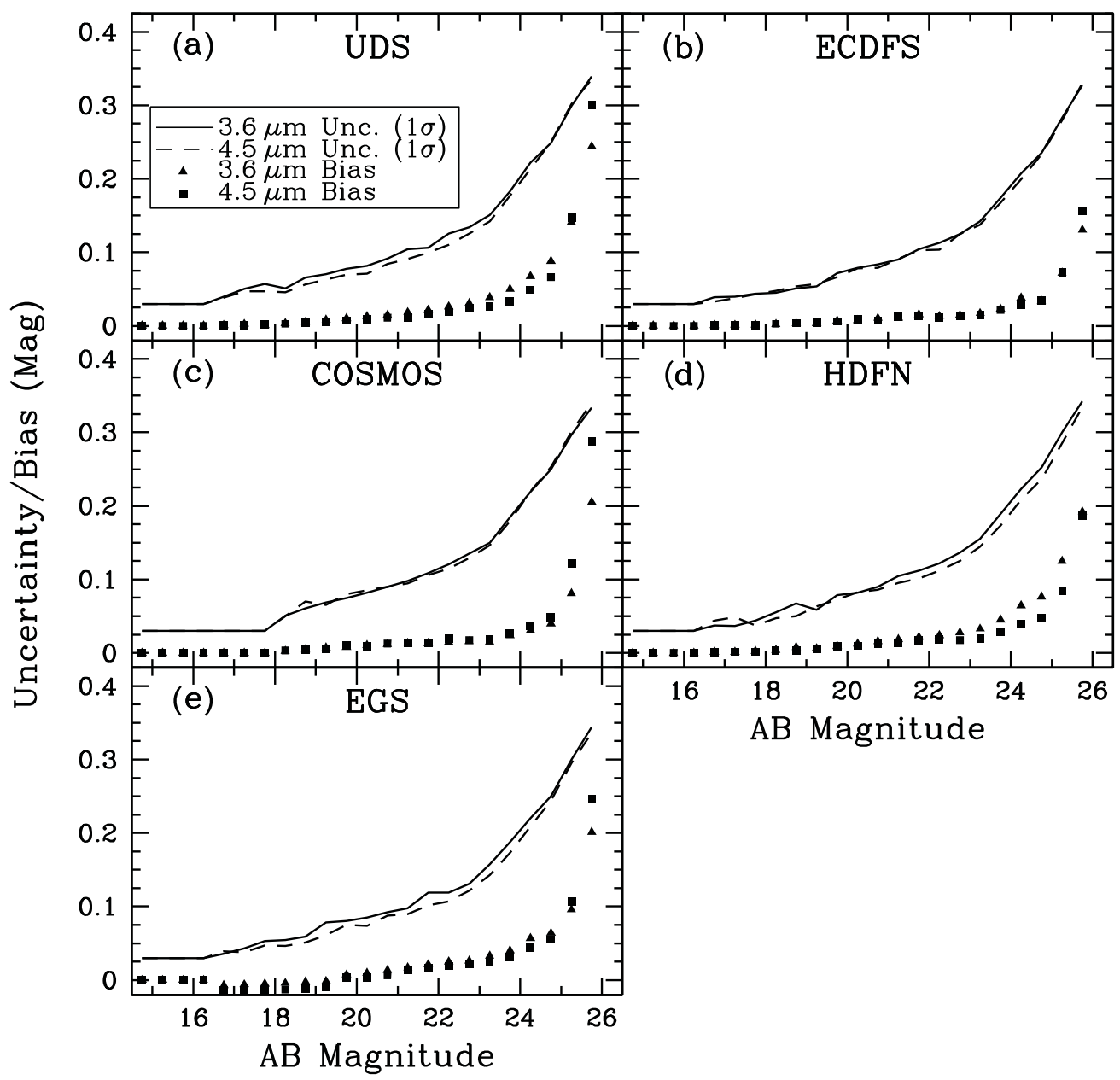

Figure 27. SEDS measurement errors based on photometry of simulated sources as described in Section 4.2. The symbols indicate the measurement bias as a function of apparent magnitude. The solid and dashed lines indicate the $1 \sigma$ measurement uncertainty at 3.6 and $4.5 \mu \mathrm{m}$, respectively. The lower limit of 0.03 mag on the measurement uncertainties reflects current knowledge of the uncertainty in the IRAC absolute calibration.

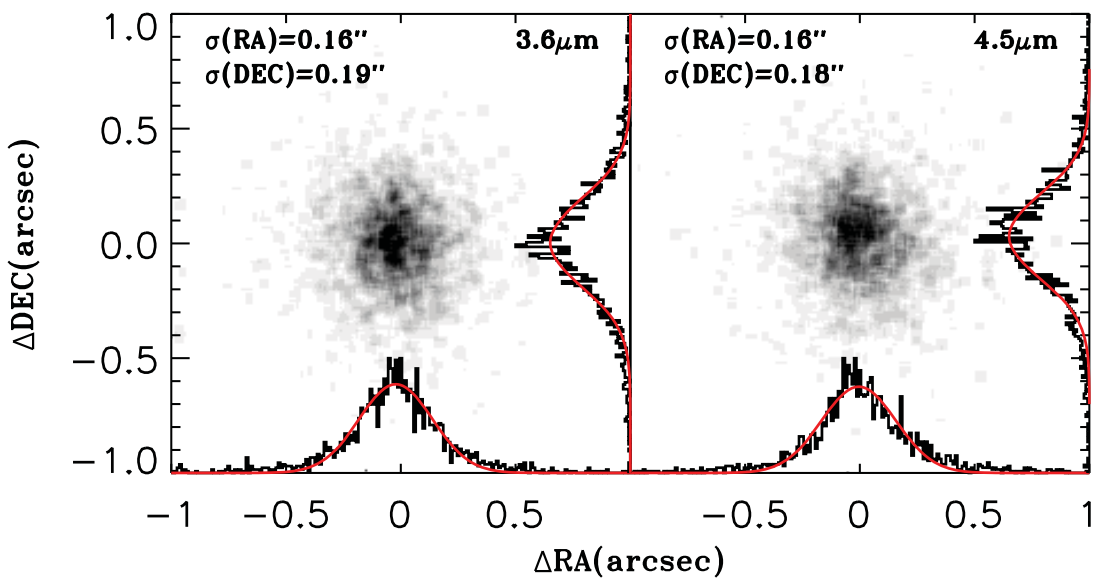

Figure 28. Left panel: Astrometric offsets (SEDS-2MASS) measured between unsaturated SEDS sources and their counterparts in the 2MASS Point Source Catalog. A $1^{\prime \prime}$ search radius was used to match roughly 2500 2MASS sources ( $\sim 500$ objects per SEDS field) with objects detected by IRAC at $3.6 \mu$ m. A high density of matches in this space is indicated with black. Gaussian functions (red) were fitted to the one-dimensional histograms of the offset distributions to estimate the $1 \sigma$ uncertainties, which are $0^{\prime \prime} 16$ in right ascension and $0^{\prime \prime} 19$ in declination. Right panel: The distribution for matched $4.5 \mu \mathrm{m}$ SEDS sources. The $1 \sigma$ uncertainties derived from fitting a Gaussian profile to the histograms of offsets are 0 !' 16 in right ascension and 0 "' 18 in declination.

(A color version of this figure is available in the online journal.)

opposite sides of the 18 mag threshold. Whereas sources fainter than 18 mag are typically galaxies, objects brighter than 18 mag are much more likely to be Galactic stars, i.e., point sources (Arendt et al. 1998; Fazio et al. 2004a; see also Figure 25).
Applying identical aperture corrections to both populations most likely introduced a slight bias to the SEDS 18-21 mag photometry of a few percent. This bias will not be significant for faint sources, because they are pointlike (Figure 25). 
Table 4

Empirical Photometric Uncertainties for SEDS

\begin{tabular}{|c|c|c|c|c|c|}
\hline Mag & UDS & ECDFS & COSMOS & HDFN & EGS \\
\hline \multicolumn{6}{|c|}{$3.6 \mu \mathrm{m}$} \\
\hline 16.25 & 0.03 & 0.03 & 0.03 & 0.03 & 0.03 \\
\hline 16.75 & 0.04 & 0.03 & 0.03 & 0.04 & 0.03 \\
\hline 17.25 & 0.05 & 0.05 & 0.03 & 0.04 & 0.03 \\
\hline 17.75 & 0.06 & 0.03 & 0.03 & 0.04 & 0.05 \\
\hline 18.25 & 0.05 & 0.04 & 0.03 & 0.07 & 0.07 \\
\hline 18.75 & 0.07 & 0.06 & 0.03 & 0.06 & 0.07 \\
\hline 19.25 & 0.07 & 0.04 & 0.06 & 0.05 & 0.07 \\
\hline 19.75 & 0.08 & 0.07 & 0.08 & 0.08 & 0.08 \\
\hline 20.25 & 0.08 & 0.08 & 0.08 & 0.08 & 0.08 \\
\hline 20.75 & 0.09 & 0.09 & 0.09 & 0.09 & 0.09 \\
\hline 21.25 & 0.10 & 0.09 & 0.10 & 0.10 & 0.10 \\
\hline 21.75 & 0.11 & 0.10 & 0.11 & 0.11 & 0.12 \\
\hline 22.25 & 0.13 & 0.11 & 0.12 & 0.12 & 0.12 \\
\hline 22.75 & 0.13 & 0.13 & 0.14 & 0.14 & 0.13 \\
\hline 23.25 & 0.15 & 0.14 & 0.15 & 0.16 & 0.16 \\
\hline 23.75 & 0.18 & 0.17 & 0.18 & 0.19 & 0.19 \\
\hline 24.25 & 0.22 & 0.21 & 0.22 & 0.22 & 0.22 \\
\hline 24.75 & 0.25 & 0.24 & 0.25 & 0.25 & 0.25 \\
\hline 25.25 & 0.30 & 0.28 & 0.30 & 0.30 & 0.30 \\
\hline 25.75 & 0.34 & 0.33 & 0.33 & 0.34 & 0.34 \\
\hline \multicolumn{6}{|c|}{$4.5 \mu \mathrm{m}$} \\
\hline 17.75 & 0.03 & 0.03 & 0.03 & 0.03 & 0.03 \\
\hline 18.25 & 0.03 & 0.03 & 0.04 & 0.03 & 0.03 \\
\hline 18.75 & 0.03 & 0.03 & 0.06 & 0.03 & 0.03 \\
\hline 19.25 & 0.07 & 0.03 & 0.07 & 0.03 & 0.03 \\
\hline 19.75 & 0.07 & 0.07 & 0.08 & 0.08 & 0.07 \\
\hline 20.25 & 0.07 & 0.08 & 0.08 & 0.08 & 0.08 \\
\hline 20.75 & 0.08 & 0.08 & 0.09 & 0.09 & 0.09 \\
\hline 21.25 & 0.09 & 0.09 & 0.10 & 0.09 & 0.09 \\
\hline 21.75 & 0.10 & 0.10 & 0.11 & 0.10 & 0.10 \\
\hline 22.25 & 0.11 & 0.10 & 0.11 & 0.11 & 0.11 \\
\hline 22.75 & 0.13 & 0.12 & 0.13 & 0.13 & 0.12 \\
\hline 23.25 & 0.14 & 0.14 & 0.15 & 0.14 & 0.14 \\
\hline 23.75 & 0.18 & 0.17 & 0.18 & 0.17 & 0.17 \\
\hline 24.25 & 0.21 & 0.20 & 0.22 & 0.21 & 0.21 \\
\hline 24.75 & 0.25 & 0.23 & 0.25 & 0.24 & 0.24 \\
\hline 25.25 & 0.30 & 0.28 & 0.30 & 0.29 & 0.30 \\
\hline 25.75 & 0.34 & 0.33 & 0.34 & 0.33 & 0.34 \\
\hline
\end{tabular}

Notes. Empirically determined $1 \sigma$ photometric uncertainties (magnitudes) determined using the Monte Carlo simulations described in Section 4.2. An estimated $3 \%$ systematic error in the IRAC flux calibration is included and limits the uncertainties for bright sources.

Several factors could account for the offsets. Changes in the IRAC calibration during the Spitzer mission could introduce changes no larger than $2 \%$. The discrepancy already noted for the SIMPLE $4.5 \mu \mathrm{m}$ band may in part be a result of the slightly different flux calibrations applied to the two SIMPLE epochs. However, a more likely cause for the discrepancies is the very different methods used to obtain the photometry in the first place. Whereas SEDS is based on a modified StarFinder approach, the SpUDS, S-COSMOS, EGS, and SIMPLE measurements are all based on SExtractor. In addition, the different surveys measured their photometry in different apertures; the comparisons presented in Figure 29 used aperture diameters of 3".8 (SpUDS), 2".8 (S-COSMOS), 4".2 (EGS), and 4".0 (SIMPLE). Even small differences in applied aperture corrections could potentially introduce the offsets seen here.

In summary, the comparison to earlier, coextensive surveys shows that SEDS faint-source photometry is generally
Table 5

Photometric Bias in SEDS Catalogs

\begin{tabular}{|c|c|c|c|c|c|}
\hline Mag & UDS & ECDFS & COSMOS & HDFN & EGS \\
\hline \multicolumn{6}{|c|}{$3.6 \mu \mathrm{m}$} \\
\hline 16.25 & 0.00 & 0.00 & 0.00 & 0.00 & 0.00 \\
\hline 16.75 & 0.00 & 0.00 & 0.00 & 0.00 & -0.01 \\
\hline 17.25 & 0.00 & 0.01 & 0.00 & 0.00 & -0.01 \\
\hline 17.75 & 0.00 & 0.00 & 0.00 & 0.00 & -0.01 \\
\hline 18.25 & 0.00 & 0.00 & 0.00 & 0.00 & -0.01 \\
\hline 18.75 & 0.00 & 0.00 & 0.00 & 0.01 & 0.00 \\
\hline 19.25 & 0.01 & 0.00 & 0.00 & 0.01 & 0.00 \\
\hline 19.75 & 0.01 & 0.01 & 0.01 & 0.01 & 0.01 \\
\hline 20.25 & 0.01 & 0.01 & 0.01 & 0.01 & 0.01 \\
\hline 20.75 & 0.01 & 0.01 & 0.01 & 0.02 & 0.01 \\
\hline 21.25 & 0.02 & 0.01 & 0.01 & 0.02 & 0.02 \\
\hline 21.75 & 0.02 & 0.02 & 0.02 & 0.02 & 0.02 \\
\hline 22.25 & 0.03 & 0.01 & 0.01 & 0.02 & 0.02 \\
\hline 22.75 & 0.03 & 0.01 & 0.02 & 0.03 & 0.03 \\
\hline 23.25 & 0.04 & 0.02 & 0.02 & 0.03 & 0.03 \\
\hline 23.75 & 0.05 & 0.02 & 0.02 & 0.05 & 0.04 \\
\hline 24.25 & 0.07 & 0.04 & 0.03 & 0.06 & 0.06 \\
\hline 24.75 & 0.09 & 0.03 & 0.04 & 0.08 & 0.06 \\
\hline 25.25 & 0.14 & 0.07 & 0.08 & 0.12 & 0.10 \\
\hline 25.75 & 0.24 & 0.13 & 0.21 & 0.19 & 0.20 \\
\hline \multicolumn{6}{|c|}{$4.5 \mu \mathrm{m}$} \\
\hline 16.25 & 0.00 & 0.00 & 0.00 & 0.00 & 0.00 \\
\hline 16.75 & 0.00 & 0.00 & 0.00 & 0.00 & -0.01 \\
\hline 17.25 & 0.00 & 0.00 & 0.00 & 0.00 & -0.01 \\
\hline 17.75 & 0.00 & 0.00 & 0.00 & 0.00 & -0.01 \\
\hline 18.25 & 0.00 & 0.00 & 0.00 & 0.00 & -0.01 \\
\hline 18.75 & 0.00 & 0.00 & 0.00 & 0.00 & -0.01 \\
\hline 19.25 & 0.01 & 0.01 & 0.01 & 0.01 & -0.01 \\
\hline 19.75 & 0.01 & 0.01 & 0.01 & 0.01 & 0.01 \\
\hline 20.25 & 0.01 & 0.01 & 0.01 & 0.01 & 0.01 \\
\hline 20.75 & 0.01 & 0.01 & 0.01 & 0.01 & 0.01 \\
\hline 21.25 & 0.01 & 0.01 & 0.01 & 0.01 & 0.01 \\
\hline 21.75 & 0.02 & 0.01 & 0.01 & 0.02 & 0.02 \\
\hline 22.25 & 0.02 & 0.01 & 0.02 & 0.02 & 0.02 \\
\hline 22.75 & 0.02 & 0.02 & 0.02 & 0.02 & 0.02 \\
\hline 23.25 & 0.03 & 0.02 & 0.02 & 0.02 & 0.02 \\
\hline 23.75 & 0.03 & 0.02 & 0.03 & 0.03 & 0.03 \\
\hline 24.25 & 0.05 & 0.03 & 0.04 & 0.04 & 0.04 \\
\hline 24.75 & 0.07 & 0.04 & 0.05 & 0.05 & 0.05 \\
\hline 25.25 & 0.15 & 0.08 & 0.12 & 0.08 & 0.11 \\
\hline 25.75 & 0.30 & 0.21 & 0.29 & 0.19 & 0.25 \\
\hline
\end{tabular}

Notes. Mean photometric bias in SEDS fields (magnitudes), determined empirically by the Monte Carlo simulations described in Section 4.2. The sense of the bias is that artificial sources are measured to be brighter, on average, than they were a priori known to be by the stated amounts. These biases have already been corrected in the catalogs presented here.

Table 6

Astrometric Offsets (SEDS-USNOB)

\begin{tabular}{lccc}
\hline \hline Field & $\begin{array}{c}\Delta \text { R.A. } \\
(\operatorname{arcsec})\end{array}$ & \multicolumn{1}{c}{$\begin{array}{c}\Delta \text { Decl. } \\
(\operatorname{arcsec})\end{array}$} & $\begin{array}{c}\text { Total } \\
(\operatorname{arcsec})\end{array}$ \\
\hline UDS & $0.09 \pm 0.19$ & $0.00 \pm 0.18$ & $0.24 \pm 0.12$ \\
ECDFS & $0.08 \pm 0.20$ & $-0.03 \pm 0.19$ & $0.27 \pm 0.12$ \\
COSMOS & $-0.02 \pm 0.19$ & $-0.11 \pm 0.17$ & $0.25 \pm 0.12$ \\
HDFN & $0.18 \pm 0.24$ & $-0.28 \pm 0.26$ & $0.44 \pm 0.19$ \\
EGS & $-0.04 \pm 0.25$ & $-0.27 \pm 0.25$ & $0.39 \pm 0.20$ \\
\hline
\end{tabular}

Notes. Mean coordinate offsets measured between SEDS sources and their visible-wavelength counterparts in the USNOB1.0 catalog (Monet et al. 2003). Total offsets refer to the mean absolute offsets between SEDS and USNOB positions. The stated uncertainties are the standard deviations of the offset distributions for matched sources. 
Table 7

SEDS Full-depth Source Catalog for the UDS Field

\begin{tabular}{|c|c|c|c|c|c|}
\hline Object & $\begin{array}{l}\text { R.A., Decl. } \\
\text { (J2000) }\end{array}$ & $\begin{array}{l}3.6 \mu \mathrm{m} \text { Magnitudes }{ }^{\mathrm{a}} \\
4.5 \mu \mathrm{m} \text { Magnitudes }\end{array}$ & $\begin{array}{l}3.6 \mu \mathrm{m} \text { Unc. }^{\mathrm{b}} \\
4.5 \mu \mathrm{m} \text { Unc. }\end{array}$ & $\begin{array}{l}3.6 \mu \mathrm{m} \mathrm{Cvg}^{\mathrm{c}} \\
4.5 \mu \mathrm{m} \mathrm{Cvg}^{\mathrm{e}}\end{array}$ & $\begin{array}{l}3.6 \mu \mathrm{m} \mathrm{Flag}{ }^{\mathrm{d}} \\
4.5 \mu \mathrm{m} \mathrm{Flag}\end{array}$ \\
\hline \multirow[t]{2}{*}{ SEDS J021641.84-051639.5 } & $34.17434-5.27763$ & 13.9513 .9413 .7713 .6813 .6313 .6113 .57 & 0.03 & 149 & 1 \\
\hline & & 14.1114 .0713 .9713 .9013 .8813 .8713 .84 & 0.03 & 0 & 3 \\
\hline \multirow[t]{2}{*}{ SEDS J021717.25-050411.5 } & $34.32189-5.06986$ & 13.9813 .9413 .6613 .5013 .4013 .3613 .27 & 0.03 & 176 & 1 \\
\hline & & 13.7713 .6913 .5213 .4213 .3713 .3613 .32 & 0.03 & 152 & 1 \\
\hline \multirow[t]{2}{*}{ SEDS J021737.30-045221.4 } & $34.40543-4.87262$ & 14.0013 .9913 .8713 .8113 .7813 .7613 .73 & 0.03 & 0 & 3 \\
\hline & & 14.7314 .6414 .4614 .3514 .3014 .2814 .24 & 0.03 & 0 & 3 \\
\hline \multirow[t]{2}{*}{ SEDS J021756.88-050757.4 } & $34.48698-5.13260$ & 14.0314 .0213 .8413 .7613 .7113 .6913 .65 & 0.03 & 187 & 1 \\
\hline & & 14.0814 .0513 .9613 .8913 .8613 .8613 .84 & 0.03 & 153 & 1 \\
\hline \multirow[t]{2}{*}{ SEDS J021818.17-050009.9 } & $34.57571-5.00276$ & 14.0313 .9713 .6513 .4513 .3313 .2813 .17 & 0.03 & 145 & 1 \\
\hline & & 13.7513 .6513 .4313 .3113 .2513 .2313 .18 & 0.03 & 107 & 1 \\
\hline \multirow[t]{2}{*}{ SEDS J021640.10-051740.5 } & $34.16709-5.29459$ & 14.1114 .1014 .0013 .9513 .9213 .9113 .89 & 0.03 & 135 & 1 \\
\hline & & 14.6014 .5714 .4714 .4014 .3714 .3614 .34 & 0.03 & 0 & 3 \\
\hline \multirow[t]{2}{*}{ SEDS J021740.09-050249.6 } & $34.41705-5.04712$ & 14.1214 .1114 .0013 .9413 .9213 .9013 .88 & 0.03 & 237 & 1 \\
\hline & & 14.2014 .1914 .1314 .1014 .0814 .0814 .07 & 0.03 & 179 & 1 \\
\hline \multirow[t]{2}{*}{ SEDS J021639.71-051836.8 } & $34.16547-5.31023$ & 14.2014 .2014 .1314 .1014 .0814 .0814 .07 & 0.03 & 124 & 1 \\
\hline & & 14.6014 .5814 .5114 .4614 .4514 .4414 .43 & 0.03 & 0 & 3 \\
\hline \multirow[t]{2}{*}{ SEDS J021725.24-051804.7 } & $34.35516-5.30130$ & 14.2214 .2114 .1414 .1014 .0814 .0714 .05 & 0.03 & 226 & 1 \\
\hline & & 14.3414 .3414 .3214 .3014 .2914 .2914 .28 & 0.03 & 344 & 1 \\
\hline \multirow[t]{2}{*}{ SEDS J021657.24-050801.5 } & $34.23849-5.13374$ & 14.2214 .2214 .1414 .1014 .0814 .0814 .06 & 0.03 & 274 & 1 \\
\hline & & 14.4514 .4414 .4114 .3914 .3814 .3714 .37 & 0.03 & 210 & 1 \\
\hline
\end{tabular}

Notes. The SEDS catalog of sources in the UDS field selected at both 3.6 and $4.5 \mu \mathrm{m}$ as described in the text.

a The PSF-fitted magnitude is given first, and the magnitudes given after are measured in apertures of 2". $4,33^{\prime \prime} 6,4{ }^{\prime \prime} 8,6$.'0, 7". 2 , and $12^{\prime \prime}$. 0 diameter.

b Stated errors are $1 \sigma$ uncertainties for the 2 ." 4 diameter aperture magnitudes.

${ }^{c}$ Depth of coverage expressed in terms of the number of unsaturated $100 \mathrm{~s} 3.6 \mu \mathrm{m}$ IRAC frames that observed the source.

d $3.6 \mu \mathrm{m}$ photometric quality flag, as described in Section 5. If the flag is nonzero, the photometry may be corrupted.

e Depth of coverage expressed in terms of the number of unsaturated $100 \mathrm{~s} 4.5 \mu \mathrm{m}$ IRAC frames that observed the source.

f $4.5 \mu \mathrm{m}$ photometric quality flag, as described in Section 5. If the flag is nonzero, the photometry may be corrupted.

(This table is available in its entirety in a machine-readable form in the online journal. A portion is shown here for guidance regarding its form and content.)

consistent with previous results even though the SEDS measurements were made using different software and methods and in significantly more crowded mosaics. Given the use of different photometric software with different parameterizations and the different depths of the data sets being compared, we regard the agreement between SEDS and the preexisting surveys as excellent.

\section{SEDS CATALOGS}

The SEDS IRAC catalogs are presented in Tables 7 through 11 . In addition to the PSF-fitted magnitudes on which the source detection is based, they also list the IRAC positions with the 3.6 and $4.5 \mu \mathrm{m}$ photometry in six apertures of diameters $2^{\prime \prime} .4,3^{\prime \prime} 6,4^{\prime \prime} .8,6^{\prime \prime}, 7^{\prime \prime}$. 2 , and $12^{\prime \prime}$. All the photometry has been aperture-corrected to total magnitudes assuming the sources are pointlike. It has also been adjusted to account for the empirically determined biases given in Table 5 . In other words, all catalog magnitudes represent total magnitudes if the source is unresolved by IRAC, but the magnitudes of extended sources represent only the flux inside the given aperture. As shown in Figure 25, this will affect mainly galaxies with [3.6] $<21 \mathrm{mag}$. The catalogs also provide $1 \sigma$ uncertainty estimates, but only for the 2..4 diameter aperture photometry because time constraints made it impractical to simulate photometry for all the aperture diameters. Users are encouraged to use the 2".4 aperture for photometry of faint sources. The other apertures are provided so that users can construct for themselves the curve of growth for large, extended sources.

Users should be aware of some limitations of the SEDS source catalogs, which are described here.
1. First and foremost, we do not present the multi-epoch photometry here. Catalogs corresponding to each visit to each SEDS field will be presented in a future contribution.

2. Because of the large uncertainties (random and systematic) associated with sources fainter than $26 \mathrm{AB}$ mag, the SEDS catalogs have been cut at this magnitude. Specifically, only sources detected at both 3.6 and $4.5 \mu \mathrm{m}$, and brighter than $26 \mathrm{mag}$ in at least one band, appear in the catalog. Although SEDS is sufficiently deep to detect fainter sources, the signal-to-noise ratio falls below $3 \sigma$ significance at $26 \mathrm{mag}$. Such faint sources are also subject to large systematic effects (e.g., the large systematic errors in the faint-source completeness estimates given in Table 3 dominate the uncertainties in the counts), and they are not reliably recovered in coextensive CANDELS HST/ WFC3 F160W observations. Nonetheless, at 26 AB mag the SEDS catalogs reach fainter than any other catalogs of which we are aware for a survey of this size at these wavelengths.

3. Confusion is a major issue for the SEDS catalogs, given the high source area density relative to the effective IRAC beam sizes at 3.6 and $4.5 \mu \mathrm{m}$. We require SEDS sources to be farther apart than one IRAC HWHM (roughly 0.9 ), because sources closer to each other than this could not be reliably deblended and photometered. Thus close pairs or multiples may be cataloged by SEDS as single sources.

4. Because the IRAC arrays view different areas of sky and their orientation is constrained by spacecraft visibility limits, the areas covered by the 3.6 and $4.5 \mu \mathrm{m}$ detectors do 
Table 8

SEDS Full-depth Source Catalog for the ECDFS Field

\begin{tabular}{|c|c|c|c|c|c|}
\hline Object & $\begin{array}{l}\text { R.A., Decl. } \\
\text { (J2000) }\end{array}$ & $\begin{array}{l}3.6 \mu \mathrm{m} \text { Magnitudes }^{\mathrm{a}} \\
4.5 \mu \mathrm{m} \text { Magnitudes }\end{array}$ & $\begin{array}{l}3.6 \mu \mathrm{m} \text { Unc. }^{\mathrm{b}} \\
4.5 \mu \mathrm{m} \text { Unc. }\end{array}$ & $\begin{array}{l}3.6 \mu \mathrm{m} \mathrm{Cvg}{ }^{\mathrm{c}} \\
4.5 \mu \mathrm{m} \mathrm{Cvg}{ }^{\mathrm{e}}\end{array}$ & $\begin{array}{l}3.6 \mu \mathrm{m} \mathrm{Flag}{ }^{\mathrm{d}} \\
4.5 \mu \mathrm{m} \mathrm{Flag}\end{array}$ \\
\hline \multirow[t]{2}{*}{ SEDS J033255.60-273039.4 } & $53.23165-27.51095$ & 14.4314 .3814 .2714 .2114 .1914 .1814 .15 & 0.03 & 0 & 3 \\
\hline & & 14.8814 .8214 .7014 .6214 .5814 .5714 .54 & 0.03 & 0 & 3 \\
\hline & & 15.0515 .0014 .8514 .7514 .7114 .7014 .67 & 0.03 & 130 & 0 \\
\hline \multirow[t]{2}{*}{ SEDS J033232.62-273754.0 } & $53.13592-27.63166$ & 14.4914 .4114 .2714 .2314 .2114 .2014 .15 & 0.03 & 0 & 3 \\
\hline & & 14.8614 .8114 .6314 .5414 .4914 .4714 .43 & 0.03 & 0 & 3 \\
\hline \multirow[t]{2}{*}{ SEDS J033248.11-273259.6 } & $53.20047-27.54988$ & 14.6514 .6214 .5514 .5114 .5014 .4914 .47 & 0.03 & 123 & 1 \\
\hline & & 14.9914 .9814 .9114 .8714 .8614 .8514 .83 & 0.03 & 205 & 0 \\
\hline \multirow[t]{2}{*}{ SEDS J033219.13-273933.6 } & $53.07972-27.65933$ & 14.6814 .6514 .6014 .5714 .5614 .5614 .55 & 0.03 & 195 & 1 \\
\hline & & 14.9314 .9214 .9014 .8914 .8914 .8914 .88 & 0.03 & 411 & 0 \\
\hline \multirow[t]{2}{*}{ SEDS J033315.93-280332.7 } & $53.31636-28.05909$ & 14.7014 .6714 .6314 .5914 .5714 .5614 .54 & 0.03 & 0 & 3 \\
\hline & & 14.7714 .7514 .7414 .7314 .7214 .7214 .71 & 0.03 & 234 & 1 \\
\hline \multirow[t]{2}{*}{ SEDS J033149.13-273110.7 } & $52.95469-27.51964$ & 14.7514 .7114 .6114 .5614 .5414 .5214 .50 & 0.03 & 0 & 3 \\
\hline & & 15.1715 .1115 .0515 .0114 .9914 .9914 .97 & 0.03 & 0 & 2 \\
\hline
\end{tabular}

Notes. As Table 7, but for the full-depth SEDS observations of the ECDFS.

a The PSF-fitted magnitude is given first, and the magnitudes given after are measured in apertures of 2". $4,33^{\prime \prime} 6,4{ }^{\prime \prime} 8,6$, $0,7^{\prime \prime} .2$, and $12^{\prime \prime} 0$ diameter.

${ }^{\mathrm{b}}$ Stated errors are $1 \sigma$ uncertainties for the 2 ". 4 diameter aperture magnitudes.

${ }^{c}$ Depth of coverage is expressed in terms of the number of unsaturated $100 \mathrm{~s} 3.6 \mu \mathrm{m}$ IRAC frames that observed the source.

d $3.6 \mu \mathrm{m}$ photometric quality flag, as described in Section 5. If the flag is nonzero, the photometry may be corrupted.

${ }^{\mathrm{e}}$ Depth of coverage expressed in terms of the number of unsaturated $100 \mathrm{~s} 4.5 \mu \mathrm{m}$ IRAC frames that observed the source.

f $4.5 \mu \mathrm{m}$ photometric quality flag, as described in Section 5. If the flag is nonzero, the photometry may be corrupted.

(This table is available in its entirety in a machine-readable form in the online journal. A portion is shown here for guidance regarding its form and content.)

Table 9

SEDS Full-depth Source Catalog for the COSMOS Field

\begin{tabular}{|c|c|c|c|c|c|}
\hline Object & $\begin{array}{l}\text { R.A., Decl. } \\
\text { (J2000) }\end{array}$ & $\begin{array}{l}3.6 \mu \mathrm{m} \text { Magnitudes }{ }^{\mathrm{a}} \\
4.5 \mu \mathrm{m} \text { Magnitudes }\end{array}$ & $\begin{array}{l}3.6 \mu \mathrm{m} \text { Unc. }^{\mathrm{b}} \\
4.5 \mu \mathrm{m} \text { Unc. }\end{array}$ & $\begin{array}{l}3.6 \mu \mathrm{m} \mathrm{Cvg}{ }^{\mathrm{c}} \\
4.5 \mu \mathrm{m} \mathrm{Cvg}{ }^{\mathrm{e}}\end{array}$ & $\begin{array}{l}3.6 \mu \mathrm{m} \mathrm{Flag}{ }^{\mathrm{d}} \\
4.5 \mu \mathrm{m} \mathrm{Flag}^{\mathrm{f}}\end{array}$ \\
\hline \multirow[t]{2}{*}{ SEDS J100032.58+020825.5 } & 150.135732 .14043 & 14.2314 .0613 .8213 .6413 .5213 .4813 .38 & 0.03 & 69 & 3 \\
\hline & & 14.0914 .0013 .7613 .6213 .5613 .5413 .50 & 0.03 & 85 & 3 \\
\hline & & 14.6114 .5714 .4214 .3414 .3014 .2914 .26 & 0.03 & 119 & 1 \\
\hline \multirow[t]{2}{*}{ SEDS J100036.89+022357.4 } & 150.153712 .39927 & 14.3914 .3214 .1714 .0814 .0314 .0113 .98 & 0.03 & 37 & 3 \\
\hline & & 14.7214 .6714 .5414 .4614 .4114 .4014 .36 & 0.03 & 80 & 1 \\
\hline \multirow[t]{2}{*}{ SEDS J100032.73+020505.7 } & 150.136382 .08493 & 14.4114 .3414 .1714 .0714 .0314 .0113 .97 & 0.03 & 27 & 3 \\
\hline & & 14.7214 .6814 .5214 .4214 .3814 .3614 .33 & 0.03 & 81 & 1 \\
\hline \multirow[t]{2}{*}{ SEDS J100027.70+022752.2 } & 150.115402 .46451 & 14.4414 .3914 .2014 .1214 .0814 .0714 .03 & 0.03 & 49 & 3 \\
\hline & & 14.7614 .7214 .5714 .4914 .4514 .4414 .42 & 0.03 & 109 & 1 \\
\hline \multirow[t]{2}{*}{ SEDS J100045.09+021636.8 } & 150.187872 .27690 & 14.5014 .4514 .3114 .2314 .1914 .1814 .15 & 0.03 & 38 & 1 \\
\hline & & 14.7714 .7414 .6614 .6014 .5714 .5614 .54 & 0.03 & 72 & 1 \\
\hline \multirow{2}{*}{ SEDS J100038.22+014730.0 } & 150.159271 .79166 & 14.6714 .6414 .5414 .5014 .4714 .4614 .44 & 0.03 & 145 & 1 \\
\hline & & 14.8014 .8014 .7914 .7714 .7614 .7614 .76 & 0.03 & 263 & 1 \\
\hline
\end{tabular}

Notes. As Table 7, but for the full-depth SEDS observations of the COSMOS field.

${ }^{a}$ The PSF-fitted magnitude is given first, and the magnitudes given after are measured in apertures of 2"'4, 3"'6, 4". 8,6 6. $^{\prime \prime} 0,7^{\prime \prime} .2$, and 12'.0 diameter.

b Stated errors are $1 \sigma$ uncertainties for the 2 ". 4 diameter aperture magnitudes.

${ }^{c}$ Depth of coverage is expressed in terms of the number of unsaturated $100 \mathrm{~s} 3.6 \mu \mathrm{m}$ IRAC frames that observed the source.

d $3.6 \mu \mathrm{m}$ photometric quality flag, as described in Section 5. If the flag is nonzero, the photometry may be corrupted.

${ }^{\mathrm{e}}$ Depth of coverage expressed in terms of the number of unsaturated $100 \mathrm{~s} 4.5 \mu \mathrm{m}$ IRAC frames that observed the source.

f $4.5 \mu \mathrm{m}$ photometric quality flag, as described in Section 5. If the flag is nonzero, the photometry may be corrupted.

(This table is available in its entirety in a machine-readable form in the online journal. A portion is shown here for guidance regarding its form and content.) 
Table 10

SEDS Full-depth Catalog for the HDFN

\begin{tabular}{|c|c|c|c|c|c|}
\hline Object & $\begin{array}{l}\text { R.A., Decl. } \\
\text { (J2000) }\end{array}$ & $\begin{array}{c}3.6 \mu \mathrm{m} \text { Magnitudes }{ }^{\mathrm{a}} \\
4.5 \mu \mathrm{m} \text { Magnitudes }\end{array}$ & $\begin{array}{l}3.6 \mu \mathrm{m} \mathrm{Unc.}^{\mathrm{b}} \\
4.5 \mu \mathrm{m} \text { Unc. }\end{array}$ & $\begin{array}{l}3.6 \mu \mathrm{m} \mathrm{Cvg}^{\mathrm{c}} \\
4.5 \mu \mathrm{m} \mathrm{Cvg}{ }^{\mathrm{e}}\end{array}$ & $\begin{array}{l}3.6 \mu \mathrm{m} \mathrm{Flag}{ }^{\mathrm{d}} \\
4.5 \mu \mathrm{m} \mathrm{Flag}\end{array}$ \\
\hline \multirow[t]{2}{*}{ SEDS J123508.40+615934.9 } & 188.7849961 .99303 & 14.0614 .0213 .9113 .8313 .7613 .7213 .64 & 0.03 & 4 & 3 \\
\hline & & 14.3814 .2814 .0613 .9513 .8913 .8713 .82 & 0.03 & 1 & 3 \\
\hline & & 14.6314 .5514 .4314 .3714 .3114 .2714 .20 & 0.03 & 4 & 3 \\
\hline \multirow[t]{2}{*}{ SEDS J123513.78+620640.0 } & 188.8074262 .11110 & 14.2014 .1814 .0713 .9913 .9313 .9113 .86 & 0.03 & 23 & 3 \\
\hline & & 14.6614 .5814 .4214 .3214 .2614 .2414 .21 & 0.03 & 23 & 3 \\
\hline \multirow[t]{2}{*}{ SEDS J123625.04+622115.7 } & 189.1043362 .35435 & 14.2614 .2514 .1614 .1014 .0714 .0514 .03 & 0.03 & 84 & 3 \\
\hline & & 14.8214 .7714 .6514 .5714 .5314 .5214 .49 & 0.03 & 84 & 1 \\
\hline \multirow[t]{2}{*}{ SEDS J123728.27+622450.7 } & 189.3677962 .41407 & 14.3114 .2914 .1914 .1314 .1014 .0914 .06 & 0.03 & 232 & 3 \\
\hline & & 14.8314 .7814 .6414 .5614 .5214 .5114 .48 & 0.03 & 232 & 1 \\
\hline \multirow[t]{2}{*}{ SEDS J123550.75+622608.9 } & 188.9614462 .43580 & 14.3914 .3914 .3314 .2914 .2714 .2614 .24 & 0.03 & 127 & 3 \\
\hline & & 14.9514 .8914 .7714 .6814 .6314 .6214 .59 & 0.03 & 127 & 0 \\
\hline \multirow[t]{2}{*}{ SEDS J123821.37+621905.0 } & 189.5890662 .31806 & 14.5414 .3414 .2814 .2114 .0713 .9613 .56 & 0.03 & 43 & 3 \\
\hline & & 14.7314 .6714 .6014 .5914 .5814 .5214 .35 & 0.03 & 43 & 3 \\
\hline
\end{tabular}

Notes. As Table 7, but for the full-depth SEDS observations of the HDFN.

a The PSF-fitted magnitude is given first, and the magnitudes given after are measured in apertures of 2". $4,33^{\prime \prime} 6,4{ }^{\prime \prime} 8,6$, $0,7^{\prime \prime} \cdot 2$, and $12^{\prime \prime} 0$ diameter.

${ }^{\mathrm{b}}$ Stated errors are $1 \sigma$ uncertainties for the 2 ". 4 diameter aperture magnitudes.

${ }^{c}$ Depth of coverage is expressed in terms of the number of unsaturated $100 \mathrm{~s} 3.6 \mu \mathrm{m}$ IRAC frames that observed the source.

d $3.6 \mu \mathrm{m}$ photometric quality flag, as described in Section 5. If the flag is nonzero, the photometry may be corrupted.

${ }^{\mathrm{e}}$ Depth of coverage expressed in terms of the number of unsaturated $100 \mathrm{~s} 4.5 \mu \mathrm{m}$ IRAC frames that observed the source.

f $4.5 \mu \mathrm{m}$ photometric quality flag, as described in Section 5. If the flag is nonzero, the photometry may be corrupted.

(This table is available in its entirety in a machine-readable form in the online journal. A portion is shown here for guidance regarding its form and content.)

Table 11

SEDS Full-Depth Source Catalog for the EGS

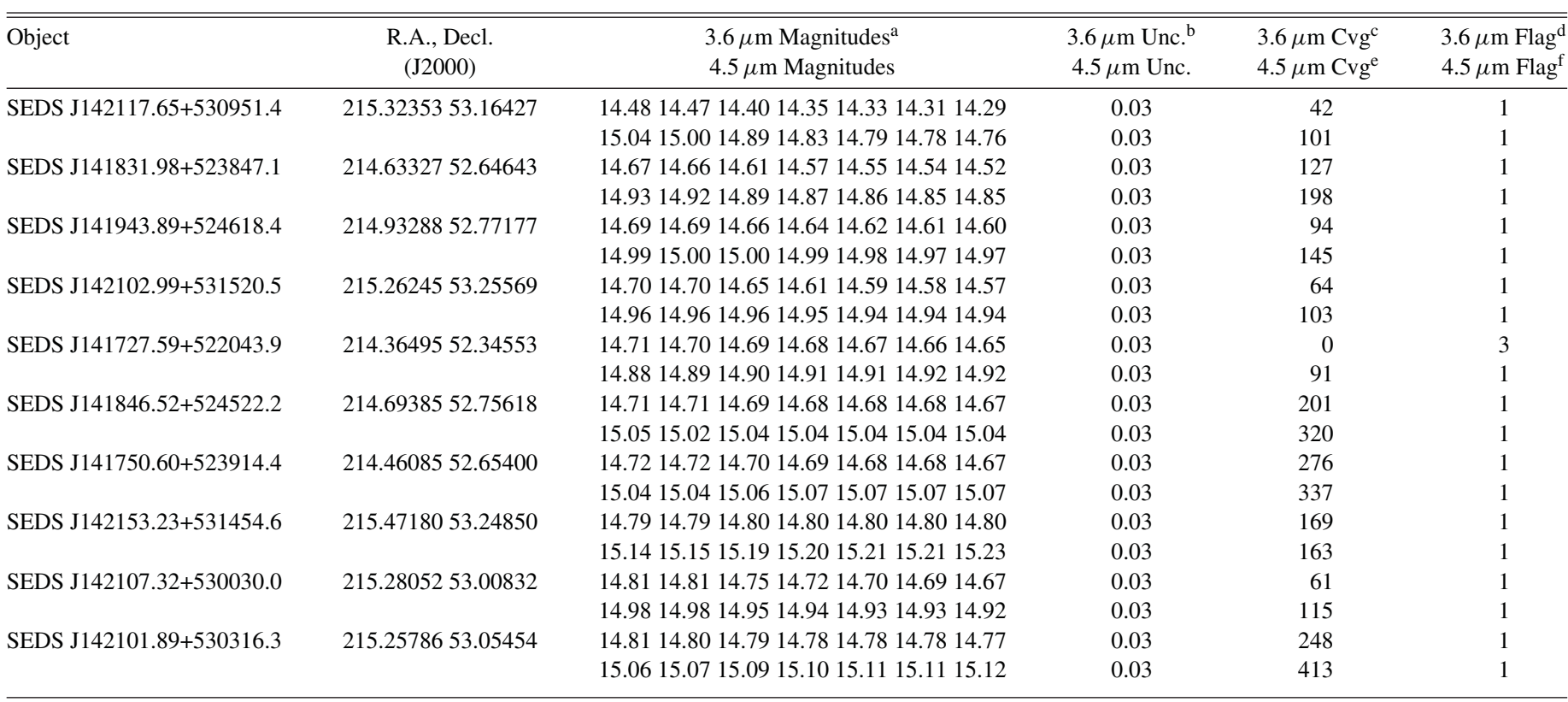

Notes. As Table 7, but for the full-depth SEDS observations of the EGS.

a The PSF-fitted magnitude is given first, and the magnitudes given after are measured in apertures of 2". $4,3^{\prime \prime} .6,44^{\prime \prime} 8,6$." $0,7^{\prime \prime} .2$, and $12^{\prime \prime} .0$ diameter.

${ }^{\mathrm{b}}$ Stated errors are $1 \sigma$ uncertainties for the 2 ". 4 diameter aperture magnitudes.

${ }^{c}$ Depth of coverage is expressed in terms of the number of unsaturated $100 \mathrm{~s} 3.6 \mu \mathrm{m}$ IRAC frames that observed the source.

d $3.6 \mu \mathrm{m}$ photometric quality flag, as described in Section 5. If the flag is nonzero, the photometry may be corrupted.

${ }^{\mathrm{e}}$ Depth of coverage expressed in terms of the number of unsaturated $100 \mathrm{~s} 4.5 \mu \mathrm{m}$ IRAC frames that observed the source.

f $4.5 \mu \mathrm{m}$ photometric quality flag, as described in Section 5. If the flag is nonzero, the photometry may be corrupted.

(This table is available in its entirety in a machine-readable form in the online journal. A portion is shown here for guidance regarding its form and content.) 


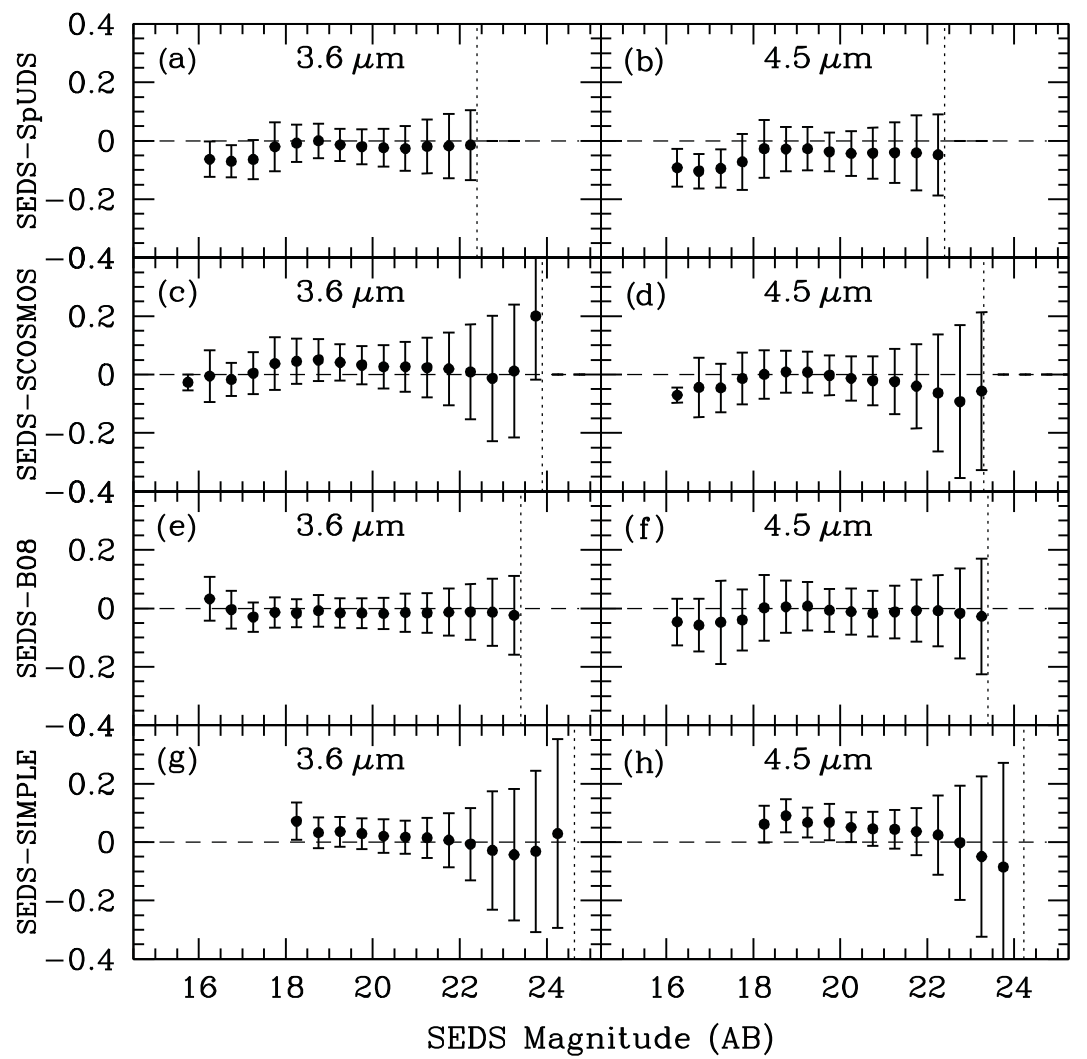

Figure 29. A comparison of SEDS 2".4 diameter aperture photometry to that of four shallower coextensive surveys from the cryogenic mission. Symbols indicate the mean differences measured within 0.5 mag bins. All error bars are $1 \sigma$. Panels (a) and (b): SEDS compared to SExtractor-based aperture photometry from SpUDS DR2. Vertical dotted lines indicate the SpUDS $5 \sigma$ sensitivity limits. Panels (c) and (d): SEDS compared to SExtractor-based aperture photometry from S-COSMOS (Sanders et al. 2007). Vertical dotted lines indicate the $1 \mu \mathrm{Jy}$ threshold of the S-COSMOS $3.6 \mu \mathrm{m}$ selection, and the $1.7 \mu \mathrm{Jy}$ S-COSMOS sensitivity at $4.5 \mu \mathrm{m}$. Panels (e) and (f): SEDS compared to the SExtractor-based aperture photometry from Barmby et al. (2008). The vertical dotted lines indicate the Barmby et al. (2008) 50\% completeness limits. Panels (g) and (h): SEDS compared to the SExtractor-based aperture photometry from SIMPLE version DR1. The vertical dotted lines indicate the $5 \sigma$ point-source depths for the deepest SIMPLE integrations $(2.5 \mathrm{hr})$.

not coincide perfectly. Irregular regions on the periphery of the SEDS fields are covered deeply by only one of the two arrays. This version of the SEDS catalogs only includes sources imaged deeply in both of the SEDS passbands.

5. The aperture photometry is likely to be corrupted for sources that fall on a strong negative residual from a relatively bright nearby source, and/or for sources that lie on or near pixels with (spurious) integration times of zero. Sources with photometry that may be corrupted owing to their proximity to at least one bright source have flags set to 1 in the catalogs. Sources with photometry that may be corrupted because of saturation have flags set to 2 in the catalogs. SEDS flags are determined separately for the 3.6 and $4.5 \mu \mathrm{m}$ mosaics, and are cumulative.

6. SEDS has been optimized specifically for faint-source photometry. Bright stars are problematic because they saturate in the 100 s SEDS exposures. Furthermore, SEDS photometry relies on a PSF-fitting technique that cannot be expected to be accurate for extended sources (nearby galaxies). Extended sources will have more flux in the larger apertures than in the smaller ones. It is recommended that users rely on the 2". 4 diameter aperture photometry for sources fainter than 21 mag because this aperture size is an appropriate match to the apparent solid angle of the faint sources that SEDS is designed to survey, and because this is also the aperture that best excludes contributions from nearby but unrelated sources in the field.

\section{DISCUSSION}

\subsection{Confusion Noise}

The SEDS uncertainties are dominated by the $3 \%$ uncertainty in the IRAC absolute calibration for sources brighter than $17 \mathrm{mag}$. They are likewise dominated by the IRAC sensitivity at the faint limit. At intermediate magnitudes, however, confusion noise is the dominant contributor to the uncertainties (Table 4). This is expected. The SEDS IRAC number counts meet the Rowan-Robinson (2001) criterion for confusion (1 source per 40 beams) at roughly $21.8 \mathrm{mag}$ in both bands. Confusion contributes to the errors even at brighter magnitudes, however. Between 18 and $25 \mathrm{mag}$, then, the uncertainties plotted in Figure 27 broadly reflect the confusion noise. Future surveys with highly redundant coverage may expect to encounter similar levels of confusion noise if their observations have the same angular resolution as SEDS.

\subsection{IRAC Color Distribution}

The IRAC colors of the sources give clues to their redshifts and luminosities. Sorba \& Sawicki (2010) and Barro et al. (2011) showed that the [3.6] - [4.5] color is a useful photometric redshift indicator, especially for separating galaxies at $z \lesssim 1.3$ from those at $z \gtrsim 1.5$. Figure 30 shows a color-magnitude diagram of the SEDS sources. As expected, sources with [3.6] < 19 have [3.6] $-[4.5] \approx-0.4$, consistent with latetype stars or low-redshift galaxies. Franceschini et al. (2006) found that galaxies in this magnitude range mostly have redshifts 


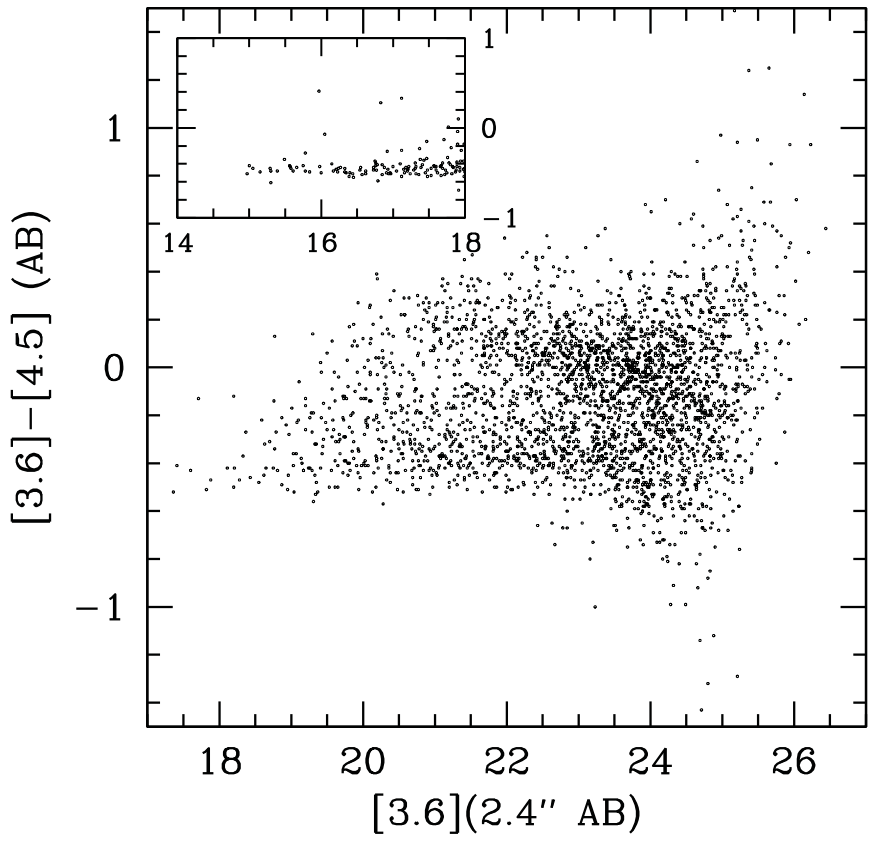

Figure 30. Color-magnitude diagram for SEDS sources. The inset shows a diagram for sources with $14<[3.6]<18$. For clarity, the main diagram only shows 1 source in 100 and the inset shows 1 in 10 . There are 302 objects $(\sim 0.1 \%$ of the catalog) with magnitudes and colors that put them outside the boundaries of both the main plot and the inset.

between 0.5 and 1.2. There are many fainter objects with similar color, but starting around [3.6] $\approx 21$, another population with [3.6] $-[4.5] \approx 0.1$ appears. This is consistent with galaxies at $z \gtrsim 1.3$ (Sorba \& Sawicki 2010). Helgason et al. (2012) derived galaxy luminosity functions (LFs) at a range of wavelengths as a function of redshift. At $z \approx 1.3$, the turnover in the rest $1.63 \mu \mathrm{m} \mathrm{LF}$, approximately corresponding to observed $3.6 \mu \mathrm{m}$, is $L^{*} \approx-24.0$. This corresponds to apparent [3.6] $\approx 21.7$, roughly a magnitude fainter than the brightest $z>1.3$ galaxies. In other words, the brightest observed galaxies with colors consistent with $z>1.3$ are roughly a magnitude brighter than $L^{*}$ at that redshift. The color trends are also shown by the histograms in Figure 31. At [3.6] $\gtrsim 23.5$, precise color information is largely lost because of photometric uncertainties, but the histogram peak at [3.6] $-[4.5] \sim-0.1$ shows that the sources are a mix of low-redshift, low-luminosity galaxies and $z \gtrsim 1.3$ galaxies with higher luminosities.

\subsection{Number Counts}

SEDS detects a total of roughly 300,000 sources in the $1.46 \mathrm{deg}^{2}$ covered by the survey. Figures 32 and 33 and Table 12 present the resulting differential source counts along with Milky Way star counts estimated from the model of Arendt et al. (1998) for the SEDS lines-of-sight. At levels brighter than roughly $18 \mathrm{AB}$ mag in both SEDS bands, the IRAC counts are consistent with the star count models. SEDS contains relatively few galaxies brighter than 18 mag. The vast majority of sources fainter than 18 mag, however, are galaxies: the contributions of Milky Way stars to the faint counts are negligible.

As shown in Figures 32(e) and 33(e), the SEDS counts agree very well with those of Fazio et al. (2004a) down to the sensitivity limit of the Barmby et al. (2008) EGS survey, despite being based on a different detection/photometry technique. They are also consistent with the deeper counts in the HDFN (Figures 32(d) and 33(d)) compiled by Magdis et al. (2008).

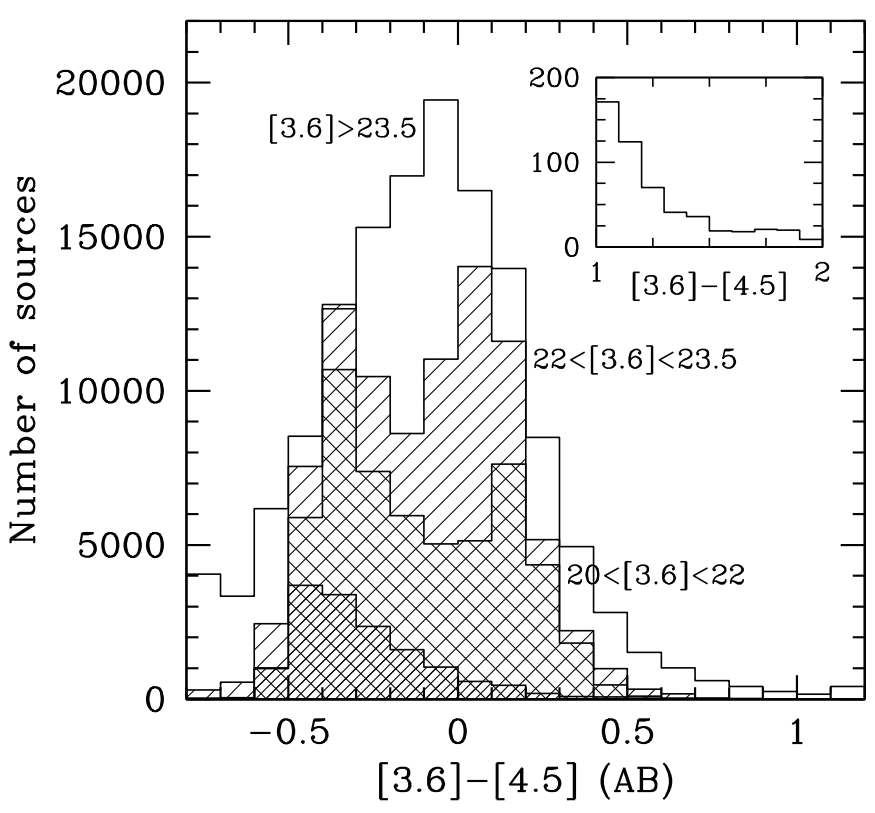

Figure 31. Histograms of SEDS source colors in four magnitude bins. The unlabeled histogram with the smallest number of sources is for [3.6] $<20$. The inset shows the color distribution of red sources $([3.6]-[4.5] \geqslant 1)$. There are 61 sources with [3.6] $-[4.5]>2$ not shown in any histogram. All but one of these have [3.6] $>$ 25.2. Magnitudes and therefore colors for sources that faint are highly uncertain.

The field-to-field variation in number counts for objects with $21<[3.6]<24$ is only $3.3 \%$ of the area-weighted mean count (Figures 34(a) and (b), and Table 12). This has significant implications for the nature of the source population. Based on tests with the QUICKCV code (Newman \& Davis 2002), such a low sample variance (or "cosmic variance" as it is commonly referred to) can only be achieved in one of two ways. One possibility is that the clustering of the sources is intrinsically weak (with a correlation length $r_{0} \sim 3 h^{-1}$ Mpc comoving, matching low-mass star-forming galaxies at $z=0-1$; Zehavi et al. 2011; Coil et al. 2008) and they have a moderately broad redshift distribution spanning $\Delta z \sim 1.5-2.5$. The other possibility is that the sources exhibit strong clustering, e.g., correlation length $r_{0} \sim 5-6 h^{-1}$ Mpc comoving, matching quiescent galaxies at $z=0-1$ (Zehavi et al. 2011; Coil et al. 2008) and Lyman-break-selected samples at $z \sim 4$ (Ouchi et al. 2004; Hildebrandt et al. 2009), but that collectively these strongly clustered sources are detected over a very wide redshift range, from $z \sim 0-4$. Subpopulations (e.g., color-selected to be at high redshift) show stronger cosmic variance than this, as would be expected if they have stronger clustering and/or span a smaller redshift range.

If a single field of the same total area as SEDS were observed, errors in the mean number density or number counts of a population due to sample/cosmic variance would be a factor of $\sim 1.25$ times higher than the errors that achieved for SEDS. ${ }^{36}$ SEDS yields lower variance because a single-field survey observes a correlated volume, which may be dominated by

\footnotetext{
36 This number was obtained by comparing the predicted errors from QUICKCV for a single square field to the predicted error in the inverse-variance-weighted (i.e., optimal/minimum-variance) mean number density resulting from a set of five fields having the actual SEDS geometries. The actual improvement depends on the total redshift range spanned by a given SEDS sample, with single-field errors ranging from 1.21 times higher than SEDS for a sample exclusively at high $z, 3.9<z<4.1$; to 1.23 times higher if the objects span $0<z<4$; to 1.39 times higher for a lower redshift range, $0<z<2$.
} 
Table 12

SEDS IRAC Number Counts

\begin{tabular}{|c|c|c|c|c|c|c|c|c|c|c|c|c|}
\hline \multirow{2}{*}{$\begin{array}{l}\text { Mag } \\
(\mathrm{AB})\end{array}$} & \multicolumn{2}{|c|}{ UDS } & \multicolumn{2}{|c|}{ ECDFS } & \multicolumn{2}{|c|}{ COSMOS } & \multicolumn{2}{|c|}{ HDFN } & \multicolumn{2}{|c|}{ EGS } & \multicolumn{2}{|c|}{ Total } \\
\hline & Counts & Unc. & Counts & Unc. & Counts & Unc. & Counts & Unc. & Counts & Unc. & Counts & Unc. \\
\hline \multicolumn{13}{|c|}{$3.6 \mu \mathrm{m}$} \\
\hline 14.75 & 2.029 & 0.243 & 2.058 & 0.224 & 2.278 & 0.236 & 2.301 & 0.200 & 1.711 & 0.333 & 2.09 & 0.32 \\
\hline 15.25 & 2.077 & 0.229 & 2.012 & 0.236 & 2.136 & 0.277 & 1.806 & 0.354 & 2.012 & 0.236 & 2.02 & 0.35 \\
\hline 15.75 & 2.246 & 0.189 & 2.188 & 0.192 & 2.499 & 0.183 & 2.079 & 0.258 & 2.137 & 0.204 & 2.23 & 0.24 \\
\hline 16.25 & 2.317 & 0.174 & 2.410 & 0.149 & 2.420 & 0.200 & 2.182 & 0.229 & 2.380 & 0.154 & 2.35 & 0.19 \\
\hline 16.75 & 2.584 & 0.128 & 2.563 & 0.125 & 2.635 & 0.156 & 2.505 & 0.158 & 2.497 & 0.135 & 2.44 & 0.14 \\
\hline 17.25 & 2.547 & 0.134 & 2.665 & 0.111 & 2.800 & 0.129 & 2.471 & 0.164 & 2.596 & 0.120 & 2.61 & 0.13 \\
\hline 17.75 & 2.723 & 0.109 & 2.864 & 0.088 & 2.925 & 0.112 & 2.812 & 0.111 & 2.726 & 0.104 & 2.81 & 0.10 \\
\hline 18.25 & 3.061 & 0.074 & 3.128 & 0.065 & 3.146 & 0.087 & 3.052 & 0.084 & 3.034 & 0.073 & 3.08 & 0.07 \\
\hline 18.75 & 3.428 & 0.048 & 3.452 & 0.045 & 3.543 & 0.055 & 3.408 & 0.056 & 3.487 & 0.043 & 3.46 & 0.04 \\
\hline 19.25 & 3.775 & 0.039 & 3.726 & 0.054 & 3.839 & 0.059 & 3.791 & 0.044 & 3.802 & 0.073 & 3.78 & 0.05 \\
\hline 19.75 & 3.974 & 0.031 & 3.980 & 0.035 & 4.052 & 0.041 & 4.014 & 0.032 & 4.024 & 0.044 & 4.01 & 0.03 \\
\hline 20.25 & 4.130 & 0.027 & 4.142 & 0.033 & 4.223 & 0.037 & 4.149 & 0.029 & 4.175 & 0.034 & 4.16 & 0.03 \\
\hline 20.75 & 4.251 & 0.025 & 4.251 & 0.032 & 4.319 & 0.037 & 4.269 & 0.026 & 4.291 & 0.032 & 4.27 & 0.03 \\
\hline 21.25 & 4.357 & 0.024 & 4.360 & 0.031 & 4.411 & 0.036 & 4.392 & 0.024 & 4.409 & 0.031 & 4.38 & 0.03 \\
\hline 21.75 & 4.476 & 0.023 & 4.466 & 0.031 & 4.502 & 0.035 & 4.491 & 0.024 & 4.514 & 0.029 & 4.49 & 0.02 \\
\hline 22.25 & 4.584 & 0.023 & 4.579 & 0.031 & 4.599 & 0.035 & 4.582 & 0.023 & 4.606 & 0.026 & 4.59 & 0.02 \\
\hline 22.75 & 4.700 & 0.023 & 4.685 & 0.021 & 4.695 & 0.022 & 4.713 & 0.023 & 4.719 & 0.026 & 4.70 & 0.02 \\
\hline 23.25 & 4.813 & 0.019 & 4.787 & 0.015 & 4.800 & 0.022 & 4.816 & 0.018 & 4.818 & 0.016 & 4.81 & 0.01 \\
\hline 23.75 & 4.892 & 0.017 & 4.883 & 0.015 & 4.880 & 0.023 & 4.908 & 0.019 & 4.896 & 0.016 & 4.89 & 0.01 \\
\hline 24.25 & 4.949 & 0.019 & 4.913 & 0.016 & 4.916 & 0.026 & 4.994 & 0.021 & 4.917 & 0.018 & 4.94 & 0.02 \\
\hline 24.75 & 4.966 & 0.048 & 4.939 & 0.047 & 4.961 & 0.040 & 5.021 & 0.036 & 4.942 & 0.052 & 4.96 & 0.04 \\
\hline 25.25 & 5.022 & 0.073 & 4.951 & 0.062 & 4.966 & 0.058 & 4.979 & 0.047 & 5.029 & 0.077 & 4.99 & 0.06 \\
\hline 25.75 & 5.115 & 0.169 & 5.031 & 0.108 & 5.206 & 0.131 & 5.025 & 0.078 & 5.110 & 0.152 & 5.09 & 0.14 \\
\hline \multicolumn{13}{|c|}{$4.5 \mu \mathrm{m}$} \\
\hline 14.75 & 1.799 & 0.302 & 1.602 & 0.378 & 2.136 & 0.277 & 1.903 & 0.289 & 1.058 & 0.707 & 1.76 & 0.50 \\
\hline 15.25 & 1.975 & 0.258 & 1.871 & 0.277 & 1.977 & 0.333 & 2.134 & 0.243 & 1.660 & 0.354 & 1.93 & 0.46 \\
\hline 15.75 & 2.160 & 0.209 & 2.079 & 0.218 & 2.136 & 0.277 & 1.944 & 0.302 & 2.099 & 0.213 & 2.09 & 0.30 \\
\hline 16.25 & 2.230 & 0.192 & 2.204 & 0.189 & 2.527 & 0.177 & 2.079 & 0.258 & 2.219 & 0.186 & 2.25 & 0.23 \\
\hline 16.75 & 2.330 & 0.171 & 2.390 & 0.152 & 2.485 & 0.186 & 2.283 & 0.204 & 2.400 & 0.151 & 2.38 & 0.18 \\
\hline 17.25 & 2.605 & 0.125 & 2.614 & 0.118 & 2.675 & 0.149 & 2.505 & 0.158 & 2.489 & 0.136 & 2.58 & 0.14 \\
\hline 17.75 & 2.674 & 0.115 & 2.721 & 0.104 & 2.897 & 0.115 & 2.556 & 0.149 & 2.716 & 0.105 & 2.71 & 0.11 \\
\hline 18.25 & 2.844 & 0.095 & 3.002 & 0.075 & 3.090 & 0.092 & 2.899 & 0.101 & 2.843 & 0.091 & 2.93 & 0.08 \\
\hline 18.75 & 3.265 & 0.058 & 3.238 & 0.057 & 3.330 & 0.070 & 3.240 & 0.068 & 3.269 & 0.055 & 3.27 & 0.05 \\
\hline 19.25 & 3.564 & 0.075 & 3.600 & 0.050 & 3.737 & 0.063 & 3.603 & 0.081 & 3.647 & 0.050 & 3.63 & 0.06 \\
\hline 19.75 & 4.069 & 0.046 & 3.894 & 0.033 & 3.993 & 0.042 & 3.946 & 0.053 & 3.979 & 0.032 & 3.98 & 0.04 \\
\hline 20.25 & 4.271 & 0.043 & 4.136 & 0.028 & 4.229 & 0.037 & 4.131 & 0.049 & 4.179 & 0.028 & 4.19 & 0.03 \\
\hline 20.75 & 4.382 & 0.042 & 4.260 & 0.027 & 4.323 & 0.036 & 4.290 & 0.048 & 4.295 & 0.028 & 4.31 & 0.03 \\
\hline 21.25 & 4.481 & 0.044 & 4.362 & 0.026 & 4.412 & 0.036 & 4.369 & 0.047 & 4.408 & 0.027 & 4.41 & 0.03 \\
\hline 21.75 & 4.579 & 0.043 & 4.445 & 0.026 & 4.488 & 0.035 & 4.462 & 0.048 & 4.493 & 0.026 & 4.50 & 0.03 \\
\hline 22.25 & 4.685 & 0.043 & 4.543 & 0.025 & 4.591 & 0.035 & 4.552 & 0.047 & 4.574 & 0.026 & 4.59 & 0.03 \\
\hline 22.75 & 4.778 & 0.044 & 4.655 & 0.015 & 4.673 & 0.023 & 4.642 & 0.049 & 4.673 & 0.018 & 4.69 & 0.03 \\
\hline 23.25 & 4.894 & 0.025 & 4.751 & 0.014 & 4.764 & 0.022 & 4.768 & 0.019 & 4.777 & 0.014 & 4.80 & 0.02 \\
\hline 23.75 & 4.975 & 0.019 & 4.841 & 0.014 & 4.856 & 0.023 & 4.842 & 0.018 & 4.849 & 0.014 & 4.88 & 0.01 \\
\hline 24.25 & 5.044 & 0.020 & 4.902 & 0.015 & 4.912 & 0.026 & 4.932 & 0.020 & 4.892 & 0.015 & 4.94 & 0.02 \\
\hline 24.75 & 5.150 & 0.047 & 4.974 & 0.046 & 5.004 & 0.039 & 5.050 & 0.028 & 4.990 & 0.052 & 5.03 & 0.04 \\
\hline 25.25 & 5.267 & 0.073 & 5.045 & 0.062 & 5.097 & 0.056 & 5.111 & 0.030 & 5.139 & 0.076 & 5.14 & 0.06 \\
\hline 25.75 & 5.158 & 0.170 & 5.179 & 0.107 & 5.112 & 0.132 & 5.237 & 0.041 & 5.128 & 0.156 & 5.17 & 0.13 \\
\hline
\end{tabular}

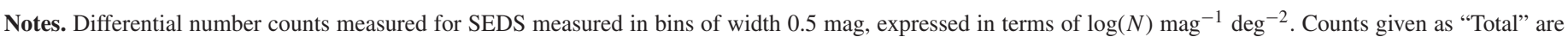

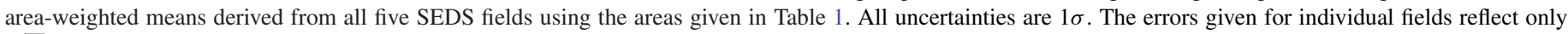
$\sqrt{N}$ counting errors, but the uncertainties attributed to "Total" counts also take field-field variations into account.

common, large-scale high- or low-density fluctuations, whereas multiple widely separated fields sample statistically independent regions of the universe. This ratio of errors is independent of linear galaxy bias, and hence of the nature of the galaxies observed.

While the ratio of errors between SEDS and a single field covering the total SEDS area is nearly independent of sample properties, the absolute reduction in errors will depend strongly on the source population's bias and redshift range. As an example, we adopt the bias predictions given in Table 4 of Moster et al. (2010) and their assumed power spectrum amplitude $\left(\sigma_{8}=0.77\right)$. In that case, a sample of galaxies with $10^{8.5} M_{\odot}<M<10^{9} M_{\odot}$ which spans $0<z<2$ would have $1.25 \%$ scatter in counts/number densities due to cosmic 


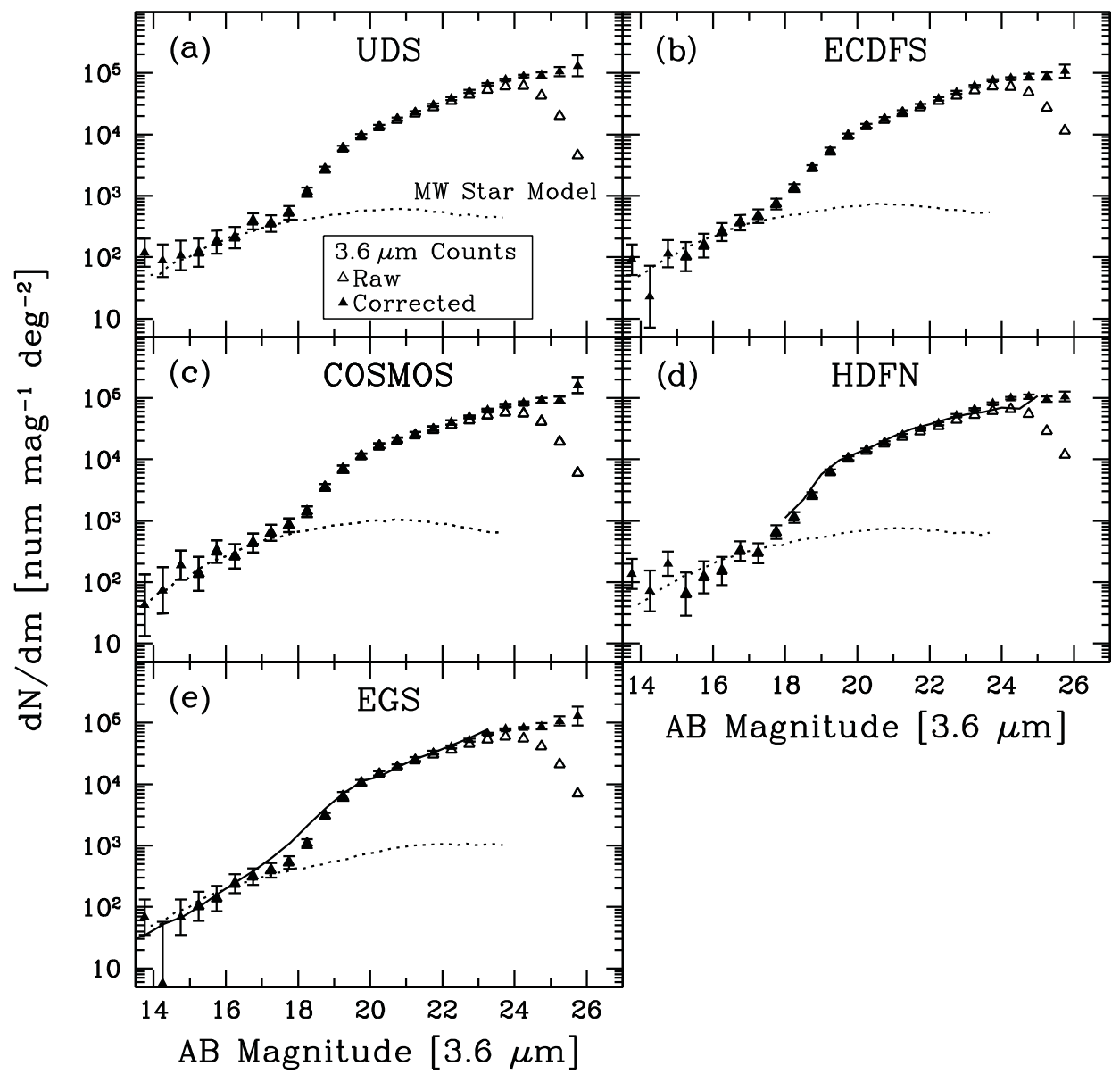

Figure 32. Differential counts in the five SEDS fields at $3.6 \mu \mathrm{m}$. Open symbols show the raw counts, while solid symbols indicate the counts corrected for incompleteness on the basis of simulated detections as described in Section 4.2. Error bars represent the Poisson statistics only. The solid lines in panels (d) and (e) indicate the incompleteness-corrected counts measured in the HDFN by Magdis et al. (2008) and in the EGS by Fazio et al. (2004a), respectively. The dotted lines in all panels are the expected counts arising from Milky Way stars along the lines of sight from the DIRBE Faint Source Model at 3.5 $\mu \mathrm{m}$ (Arendt et al. 1998; Wainscoat et al. 1992; Cohen 1993, 1994, 1995).

variance in SEDS, but would have a scatter of $1.60 \%$ in a single field of the same area. A sample with $M>10^{11} M_{\odot}$ and falling in the range $3.9<z<4.1$ would have $21.0 \%$ scatter from sample variance in SEDS, versus $25.4 \%$ in a single-field survey. To achieve the same sample/cosmic variance as SEDS, a single-field survey of the same depth would have to cover $\sim 2.4 \mathrm{deg}^{2}$, as opposed to the $1.46 \mathrm{deg}^{2}$ surveyed in the five SEDS fields. Of course, for some populations Poisson variance (shot noise) will dominate rather than sample variance; in that case only area surveyed matters. In general, the net errors in counts or number densities for a given sample from a singlefield survey must be between $0 \%$ (if Poisson-dominated) and $\sim 25 \%$ (if sample-variance-dominated) worse than if the SEDS strategy were used, given that both terms contribute to the overall error. Given that observing time is (to first order) determined by the area surveyed, a multiple-field strategy will always yield errors that are no worse than, and can be significantly better than, a single-field observation at constraining the abundances of galaxy populations.

The mean differential counts, calculated as area-weighted means, are shown in Figures 34(c) and (d). In the aggregate, the counts brighter than 18 mag in both bands very closely follow the Arendt et al. (1998) DIRBE 3.5 and $4.9 \mu \mathrm{m}$ star count models for the Milky Way. Elsewhere, the SEDS counts correspond closely to the "default model" counts of Helgason et al. (2012), who reconstruct galaxy counts in the IRAC bands from known galaxy populations using an extensive library of multi-wavelength LF measurements extending to $z \sim 4-5$.

We fitted a pure power law to the SEDS counts below the break in both bands. Specifically, we performed a $\chi^{2}$ minimization fit to the differential counts from $20-26 \mathrm{mag}$ in both SEDS bands. The fitted slopes are $0.17 \pm 0.05$ and $0.180 \pm 0.004$ at 3.6 and $4.5 \mu \mathrm{m}$, respectively, where the uncertainties are $1 \sigma$. This suggests that the dominant contributions to the faint IRAC counts come from galaxies at $z=3-5$, with an LF having a faint-end slope of roughly $\alpha=-1.5$ (Helgason et al. 2012). Although this slope is slightly lower than seen for UV LFs at similar redshifts $(\alpha \sim-1.7$; Bouwens et al. 2012; Bouwens et al. 2007), it is consistent with the trends in faintend slope documented in Helgason et al. (2012) and anchored at low redshifts by the 3.6 and $4.5 \mu \mathrm{m}$ LFs of Dai et al. (2009) and Babbedge et al. (2006).

\subsection{The Integrated Galaxy Light from IRAC Sources}

The CIB contains the sum of all light from distant galaxies integrated over all of Cosmic time, and therefore is of primary importance for understanding galaxy formation and evolution. Historically, precise measurement of the CIB has been complicated by the difficulties involved in modeling and subtracting the very substantial foreground contaminants (see, e.g., the review by Kashlinsky 2005). However, measurement of the total light from resolved sources, the integrated galaxy light 


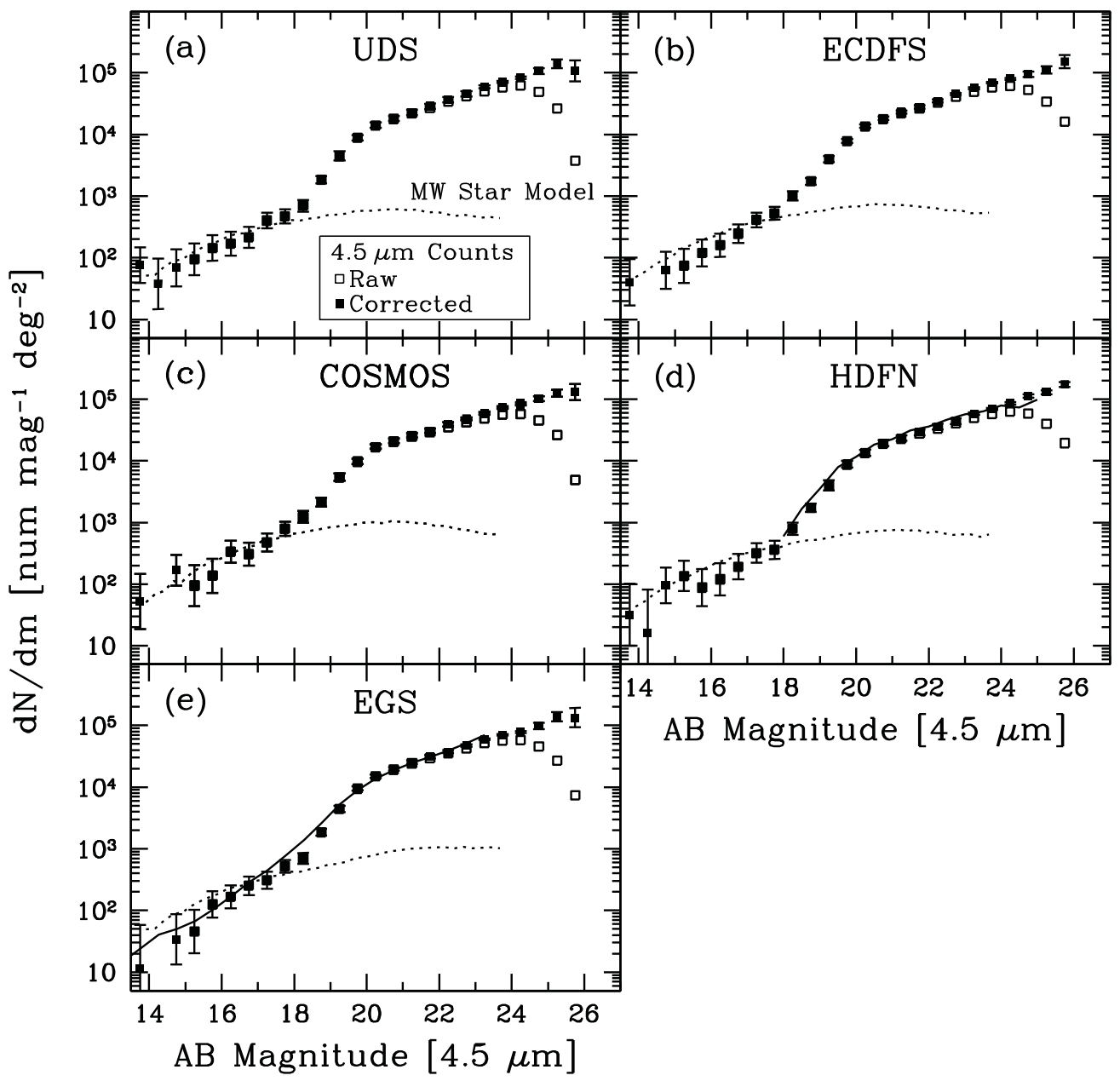

Figure 33. As Figure 32, but for the differential $4.5 \mu \mathrm{m}$ SEDS counts, and showing the DIRBE Faint Source Model counts for Milky Way stars at $4.9 \mu \mathrm{m}$.

(IGL), provides a strict and robust lower limit on the CIB and is largely unaffected by the foregrounds that complicate direct CIB measurements. Low-background space-based surveys, such as SEDS, have obvious promise for this application.

Fazio et al. (2004a) made the first measurements of the IGL with IRAC: 5.4 and $3.5 \mathrm{nW} \mathrm{m}^{-2} \mathrm{sr}^{-1}$ at 3.6 and $4.5 \mu \mathrm{m}$ respectively, down to $23.5 \mathrm{mag}$. Subsequent analysis by Kashlinsky (2005) revised these estimates respectively to $5.3 \pm 1.0$ and $4.0 \pm 0.8 \mathrm{nW} \mathrm{m}^{-2} \mathrm{sr}^{-1}$. In other words, the cryogenic IRAC observations resolved somewhat less than half of the estimated CIB at these wavelengths $\left(13.3 \pm 2.8 \mathrm{nW} \mathrm{m}^{-2} \mathrm{sr}^{-1}\right.$; Levenson et al. 2007).

The Fazio et al. (2004a) bright counts are drawn from widefield observations covering nearly $10 \mathrm{deg}^{2}$, and are therefore unlikely to suffer significantly from cosmic variance. However, as Levenson \& Wright (2008) point out, the faint Fazio et al. (2004a) counts are based on only two relatively narrow lines of sight. Levenson \& Wright (2008) therefore measured the IRAC counts toward three widely separated fields (DIRBE "Dark Spots") each covering a substantial area $\left(\sim 1 \mathrm{deg}^{2}\right)$ to estimate faint IRAC counts relatively free of cosmic variance. On the basis of an extrapolation of their counts to faint levels, Levenson \& Wright (2008) arrived at a larger estimate of the contribution of IRAC sources to the CIB: $9.0_{-0.9}^{+1.7} \mathrm{nW} \mathrm{m}^{-2} \mathrm{sr}^{-1}$.

SEDS offers a new opportunity-by virtue of its deep observations spread over five widely-separated fields - to reduce the uncertainties attending the contribution of faint IRAC sources to the CIB. SEDS is of course not optimal for bright-source count estimates. We therefore adopt the Fazio/Kashlinsky measurements for the counts brighter than $23.5 \mathrm{mag}$. Despite the fact that SEDS reaches 2.5 mag deeper than Fazio et al. (2004a), the incremental contribution of faint SEDS sources (23.5-26.0 mag) to the previous CIB estimate is small, amounting to only $\sim 10 \%$ of the earlier estimates: $0.33 \pm 0.03$ and $0.36 \pm 0.03 \mathrm{nW} \mathrm{m}^{-2} \mathrm{sr}^{-1}$ at 3.6 and $4.5 \mu \mathrm{m}$, respectively. The total contributions to the CIB of detected IRAC sources are therefore $5.6 \pm 1.0$ and $4.4 \pm 0.8 \mathrm{nW} \mathrm{m}^{-2} \mathrm{sr}^{-1}$ at 3.6 and $4.5 \mu \mathrm{m}$, respectively, down to $26 \mathrm{mag}$. Our results agree with Helgason et al. (2012), whose "default" construction estimated total contributions from IRAC sources to the CIB of $4.9_{-0.7}^{+1.7}$ and $3.3_{-0.5}^{+1.2} \mathrm{nW} \mathrm{m}^{-2} \mathrm{sr}^{-1}$ at 3.6 and $4.5 \mu \mathrm{m}$, respectively, where the ranges bracket the two extremes of the faint-end LF extrapolation consistent with the Helgason et al. (2012) LF database.

The SEDS CIB measurement is significantly smaller than that estimated in the three DIRBE "Dark Spots" at $3.6 \mu \mathrm{m}$ by Levenson \& Wright (2008). This may be because the "Dark Spots" estimate is based on an extrapolation to faint levels of relatively bright counts having a significantly steeper slope $(\sim 0.5)$ than found by SEDS. Figure 12 of Levenson \& Wright (2008) nonetheless suggests that the measured SEDS slope is consistent with their analysis at roughly the $90 \%$ confidence level.

The faint SEDS IRAC counts impose strong upper limits on the possible contribution of IRAC sources to the CIB. Even if 


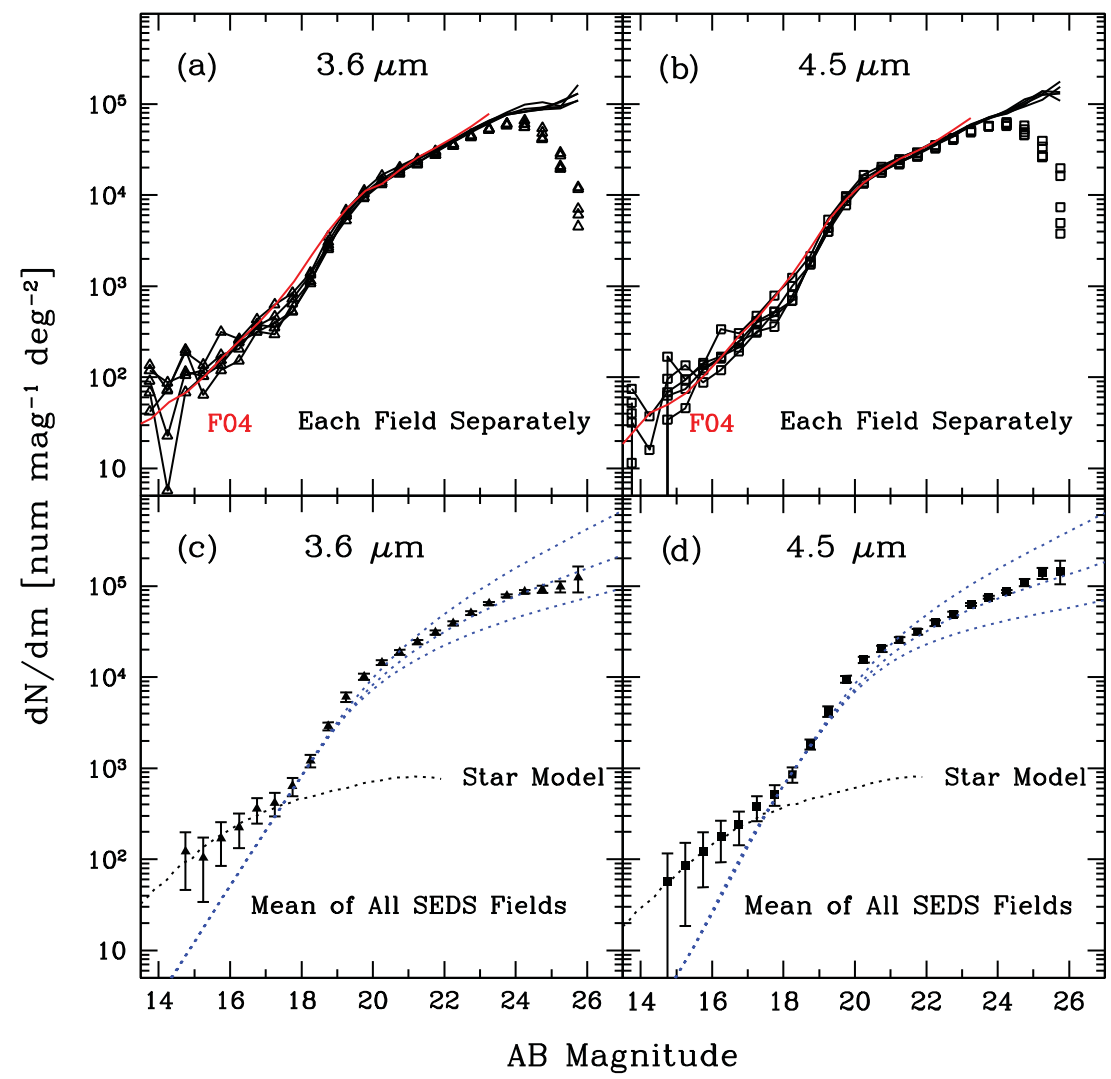

Figure 34. Differential source counts for SEDS. Panel (a): symbols indicate the $3.6 \mu \mathrm{m}$ counts in the five SEDS fields. The black lines indicate the counts after correcting for incompleteness. The red line shows the incompleteness-corrected counts from Fazio et al. (2004a). Panel (b): as panel (a), but for the 4.5 $\mu$ m counts. Panel (c): the mean differential $3.6 \mu \mathrm{m}$ source counts for all of SEDS, weighted by field area. The upper and lower dashed blue lines indicate respectively the high-faint-end (HFE) and low-faint-end (LFE) count models from Helgason et al. (2012). The dashed blue line between these two models indicates the default Helgason et al. (2012) model, which averages the HFE and LFE models. The black dotted line shows the area-weighted mean predicted Milky Way star counts based on the DIRBE $3.5 \mu \mathrm{m}$ Faint Source Model Counts (Arendt et al. 1998). Panel (d): as panel (c), but for the $4.5 \mu \mathrm{m}$ counts and showing the DIRBE Faint Source Model counts for Milky Way stars at $4.9 \mu \mathrm{m}$.

(A color version of this figure is available in the online journal.)

the slopes of the IRAC counts increased immediately below $26 \mathrm{AB}$ mag to the Helgason high faint end case ( 0.22 in both SEDS bands), the total contributions to the CIB from these undetected sources would be only 0.16 and $0.14 \mathrm{nW} \mathrm{m}^{-2} \mathrm{sr}^{-1}$ at 3.6 and $4.5 \mu \mathrm{m}$, respectively. Thus, discrete IRAC sources appear unable to furnish more than about half of the estimated $\mathrm{CIB}$ at 3.6 and $4.5 \mu \mathrm{m}$.

\subsection{Other Applications of SEDS Data}

SEDS is designed to maximize its impact in fields related to cosmology and high-redshift science. It has already been of some use in this regard. Kashlinsky et al. (2012) used SEDS imaging from two fields (the UDS and EGS) to make new measurements of fluctuations in the CIB; the SEDS data permitted extraction of much fainter sources and analysis of the CIB fluctuations over significantly larger scales than was possible previously at these wavelengths.

SEDS will be of particular interest as a means to identify distant galaxies. High-redshift galaxies have long been identified on the basis of their red colors; historically this has been accomplished in the optical-to-NIR regime (e.g., $I-K$ or $R-K$ )

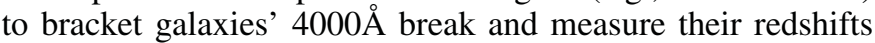
out to $z \sim 3$ (Roche et al. 2003; Franx et al. 2003; Brown et al. 2005). With Spitzer/IRAC photometry this technique has been extended out beyond $z=3$ (e.g., Wang et al. 2009; Huang et al. 2011; Wang et al. 2012). This has begun happening with
SEDS photometry as well. Aravena et al. 2010 incorporated SEDS photometry into the spectral energy distribution of a rare submm-selected galaxy in COSMOS to place it at $z \sim 4.8$. Caputi et al. (2012) identified and analyzed a sample of very rare, very red objects $(H-[4.5]>4)$ galaxies in the UDS and found that this color criterion was efficient for identifying sources at $3<z<5$. At an even more extreme redshift, Capak et al. (2011) used SEDS-COSMOS observations (along with a suite of other data) to identify a massive protocluster at $z \sim 5$.3. No doubt the SEDS data will be put to other uses not yet anticipated by the SEDS team, now that they are public.

\section{SUMMARY}

The SEDS team has carried out a deep 3.6 and $4.5 \mu \mathrm{m}$ survey of five widely separated extragalactic fields, covering a total of $1.46 \mathrm{deg}^{2}$ to a depth of $26 \mathrm{AB}$ mag $(3 \sigma)$ in both bands. The images and catalogs being made publicly available with this contribution therefore constitute a significant advance into a new regime of depth-area survey parameter space, never previously explored at these wavelengths.

SEDS has detected and photometered more than 300,000 distinct sources, of which the vast majority are galaxies. The galaxy color-magnitude distribution is consistent with mostly $z \lesssim 1.3$ galaxies at [3.6] $<21$ and a mix of low- and high-redshift galaxies at fainter magnitudes. Detailed number count models will have to reproduce both the number counts themselves and 
the color distributions as a function of magnitude. The resulting counts are consistent with but extend previous measurements to significantly fainter levels, and are consistent with infrared galaxy LFs having faint-end slopes described by power laws with indices of $\alpha=-1.5$. The contribution of resolved IRAC sources to the CIB is approximately half of the diffuse background measured by DIRBE.

The authors are grateful to E. Diolaiti for helpful advice on the optimal use of StarFinder. This work is based on observations made with the Spitzer Space Telescope, which is operated by the Jet Propulsion Laboratory, California Institute of Technology under contract with the National Aeronautics and Space Administration (NASA). Support for this work was provided by NASA through contract numbers 1367335, 1367411 and 1367412 issued by JPL/Caltech. J.S.D. acknowledges the support of the European Research Council via an Advanced Grant, and the support of the Royal Society via a Wolfson Research Merit Award. IRAF is distributed by the National Optical Astronomy Observatory, which is operated by the Association of Universities for Research in Astronomy (AURA) under cooperative agreement with the National Science Foundation.

Facilities: Spitzer (IRAC)

\section{REFERENCES}

Aravena, M., Younger, J. D., Fazio, G. G., et al. 2010, ApJL, 719, L15 Arendt, R. G., Odegard, N., Weiland, J. L., et al. 1998, ApJ, 508, 74 Ashby, M., Stern, D., Brodwin, M., et al. 2009, ApJ, 701, 428 Babbedge, T. S. R., Rowan-Robinson, M., Vaccari, M., et al. 2006, MNRAS, 370, 1159

Barmby, P., Huang, J.-S., Ashby, M. L. N., et al. 2008, ApJS, 177, 431 Barro, G., Pérez-Gonzlez, P. G., Gallego, J., et al. 2011, ApJS, 193, 30 Bertin, E., \& Arnouts, S. 1996, A\&AS, 117, 393

Bielby, R., Hudelot, P., McCracken, H. J., et al. 2012, A\&A, 545, A23 Bouwens, R. J., Illingworth, G. D., Franx, M., \& Ford, H. 2007, ApJ, 670, 928 Bouwens, R. J., Illingworth, G. D., Oesch, P. A., et al. 2012, ApJL, 752, L5

Brown, M. J. I., Jannuzi, B. T., Dey, A., \& Tiede, G. P. 2005, ApJ, 621, 41 Capak, P. L., Riechers, D., Scoville, N. Z., et al. 2011, Natur, 470, 233 Caputi, K., Cirasuolo, M., Dunlop, J. S., et al. 2011, MNRAS, 413, 162 Caputi, K., Dunlop, J. S., McLure, R. J., et al. 2012, ApJL, 750, L20 Castellano, M., Fontana, A., Boutsia, K., et al. 2010, A\&A, 511, A20 Cohen, M. 1993, AJ, 105, 1860

Cohen, M. 1994, AJ, 107, 582

Cohen, M. 1995, ApJ, 444, 874

Coil, A. L., Newman, J. A., Croton, D., et al. 2008, ApJ, 672, 153
Dai, X., Assef, R. J., Kochanek, C. S., et al. 2009, ApJ, 697, 506 Damen, M., Labbé, I., van Dokkum, P. G., et al. 2011, ApJ, 727, 1 Davé, R., Finlator, K., \& Oppenheimer, B. D. 2006, MNRAS, 370, 273 Davis, M., Guhathakurta, P., Konidaris, N. P., et al. 2007, ApJL, 660, L1 Diolaiti, E., Bendinelli, O., Bonaccini, D., et al. 2000, Proc. SPIE, 4007, 879 Fazio, G. G., Ashby, M. L. N., Barmby, P., et al. 2004a, ApJS, 154, 39 Fazio, G. G., Hora, J. L., Allen, L. E., et al. 2004b, ApJS, 154, 10 Franceschini, A., Rodighiero, G., Cassata, P., et al. 2006, A\&A, 453, 397 Franx, M., Labbé, I., Rudnick, G., et al. 2003, ApJL, 587, L79 Giavalisco, M., Ferguson, H. C., Koekemoer, A. M., et al. 2004, ApJL, 600, L93 Grogin, N. A., Kocevski, D. D., Faber, S. M., et al. 2011, ApJS, 197, 35 Hathi, N. P., Mobasher, B., Capak, P., Wang, W.-H., \& Ferguson, H. C. 2012, ApJ, 757, 43

Helgason, K., Ricotti, M., \& Kashlinsky, A. 2012, ApJ, 752, 113 Hildebrandt, H., Pielorz, J., Erben, T., et al. 2009, A\&A, 498, 725 Huang, J.-S., Zheng, X. Z., Rigopoulou, D., et al. 2011, ApJL, 742, L13 Kashlinsky, A. 2005, PhR, 409, 361

Kashlinsky, A., Arendt, R. G., Ashby, M. L. N., et al. 2012, ApJ, 753, 63 Koekemoer, A. M., Aussel, H., Calzetti, D., et al. 2007, ApJS, 172, 196 Koekemoer, A. M., Faber, S. M., Ferguson, H. C., et al. 2011, ApJS, 197, 36 Labbé, I., Oesch, P. A., Bouwens, R. J., et al. 2012, arXiv:1209.3037 Lawrence, A., Warren, S. J., Almaini, O., et al. 2007, MNRAS, 379, 1599 Levenson, L. R., \& Wright, E. L. 2008, ApJ, 683, 585

Levenson, L. R., Wright, E. L., \& Johnson, B. D. 2007, ApJ, 666, 34 Lin, L., Dickinson, M., Jian, H.-Y., et al. 2012, ApJ, 756, 71 Lonsdale, C. J., Polletta, M. d. C., Surace, J., et al. 2004, ApJS, 154, 54 Lonsdale, C. J., Smith, H. E., Rowan-Robinson, M., et al. 2003, PASP, 115, 897 Magdis, G. E., Rigopoulou, D., Huang, J.-S., et al. 2008, MNRAS, 386, 11 McCracken, H. J., Milvang-Jensen, B., Dunlop, J., et al. 2012, A\&A, 544, A156 Monet, D. G., Levine, S. E., Canzian, B., et al. 2003, AJ, 125, 984 Moster, B. P., Somerville, R. S., Maulbetsch, C., et al. 2010, ApJ, 710, 903 Newman, J. A., \& Davis, M. 2002, ApJ, 564, 567

Ouchi, M., Shimasaku, K., Okamura, S., et al. 2001, ApJL, 558, L83

Ouchi, M., Shimasaku, K., Okamura, S., et al. 2004, ApJ, 611, 685

Reach, W. T., Megeath, S. T., Cohen, M., et al. 2005, PASP, 117, 978

Rix, H.-W., Barden, M., Beckwith, S. V. W., et al. 2004, ApJS, 152, 163

Roche, N. D., Dunlop, J., \& Almaini, O. 2003, MNRAS, 346, 803

Rowan-Robinson, M. 2001, ApJ, 549, 745

Sanders, D. B., Salvato, M., Aussel, H., et al. 2007, ApJS, 172, 86 Schuster, M. T., Marengo, M., \& Patten, B. M. 2006, Proc. SPIE, 6270, 65

Scoville, N., Abraham, R. G., Aussel, H., et al. 2007a, ApJS, 172, 38 Scoville, N., Aussel, H., Brusa, M., et al. 2007b, ApJS, 172, 1

Skrutskie, M. F., Cutri, R. M., Stiening, R., et al. 2006, AJ, 131, 1163

Sorba, R., \& Sawicki, M. 2010, ApJ, 721, 1056

Wainscoat, R., Cohen, M., Volk, K., et al. 1992, ApJS, 83, 111

Wang, W.-H., Barger, A. J., \& Cowie, L. L. 2009, ApJ, 690, 319

Wang, W.-H., Barger, A. J., \& Cowie, L. L. 2012, ApJ, 744, 155

Wang, W.-H., Cowie, L. L., Barger, A. J., Keenan, R. C., \& Ting, H.-C. 2010, ApJS, 187, 251

Werner, M. W., Roellig, T. L., Low, F. J., et al. 2004, ApJS, 154, 1 Williams, R. E., Blacker, B., Dickinson, M., et al. 1996, AJ, 112, 1335

Zehavi, I., Zheng, Z., Weinberg, D. H., et al. 2011, ApJ, 736, 59 